\title{
The socio-endocrinology of female reproductive strategies in wild Assamese macaques (Macaca assamensis)
}

\author{
Dissertation \\ zur Erlangung des mathematisch-naturwissenschaftlichen \\ Doktorgrades \\ "Doctor rerum naturalium" \\ der Georg-August-Universität Göttingen
}

vorgelegt von

Ines Fürtbauer

aus Wien, Österreich

Göttingen 2011 
Referentin: Prof. Dr. Julia Ostner Korreferent: Prof. Dr. Peter M. Kappeler Tag der mündlichen Prüfung: 4. Juli 2011 
Table of contents

GENERAL INTRODUCTION

\section{CHAPTER 1:}

Reproductive and life history parameters of wild female Macaca assamensis

with O. Schülke, M. Heistermann \& J. Ostner

\section{CHAPTER 2:}

Fecal androgen excretion and fetal sex effects during gestation in wild Assamese macaques (Macaca assamensis)

with M. Heistermann, O. Schülke \& J. Ostner

27

\section{CHAPTER 3:}

Concealed fertility and extended female sexuality in a non-human primate

(Macaca assamensis)

with M. Heistermann, O. Schülke \& J. Ostner

39

\section{CHAPTER 4:}

You mate, I mate: Macaque females synchronize sex not cycles 



\section{GENERAL INTRODUCTION}

\section{Sexually antagonistic strategies \& intersexual conflict}

Sexual selection (Darwin, 1871) arises from intrasexual competition, mate choice, and sexual coercion (e.g. Smuts \& Smuts, 1993), and drives the evolution of certain traits (i.e. ornaments, signals, larger body size, and weapons) and sex-specific reproductive strategies (Darwin, 1871). Generally, the sex that invests less in offspring will compete over mating access to the other sex (Bateman, 1948; Trivers, 1972). Owing to their long gestation and lactation periods, parental investment in mammals is usually biased towards females (Trivers, 1972). Hence, females have lower potential reproductive rates (Clutton-Brock \& Vincent, 1991), and are a limiting resource for males, leading to a skewed operational sex ratio (OSR, Emlen \& Oring, 1977). Accordingly, males will attempt to monopolize reproduction in order to fertilize as many females as possible whereas females should be more selective of their mates (Bateman, 1948; Trivers, 1972). This, however, leads to conflicting interests among males and females, resulting in a coevolutionary arms race between the sexes, with each sex continually evolving counterstrategies to oppose those of the other sex (Trivers, 1972; for review see Arnquist \& Rowe, 2005).

Infanticide by males, a form of male sexual coercion, represents a significant source of intersexual conflict in vertebrates (e.g. van Schaik \& Janson, 2000; Wolff \& Mcdonald, 2004). Primates, in particular, are highly vulnerable to infanticide owing to their high lactation to gestation ratio (e.g. van Noordwijk \& van Schaik, 2000; van Schaik, 2000a). Infanticide shortens interbirth intervals and may accelerate a female's subsequent conception, hence is an adaptive male strategy provided that the male did not sire the infant. Male primates cannot directly recognize their own infants (but see Buchan et al., 2003) and thus, infanticide "decisions" are based on paternity estimates, i.e. the mating history with the mother (e.g. Soltis, 2000; van Schaik et al., 2004).

In the face of risk of infanticide, females have evolved numerous counterstrategies to manipulate male paternity estimates, i.e. they exhibit ways to (i) manipulate the distribution of paternity among males, and to (ii) manipulate the information available to males on which to base their paternity estimates (e.g. Clarke et al., 2009; van Schaik, 2000b; van Schaik et al., 1999, 2000, 2004). By mating promiscuously, i.e. providing many males with a non-zero probability of paternity, females may decrease the risk of infanticide by males (Borries et al., 1999; Hrdy, 1979; van Schaik, 2000b; van Schaik et al., 1999; Soltis et al., 2000). Additionally, promiscuous mating may secure or increase male care for future infants (e.g. van Schaik et al., 2000, 2004; Soltis, 2002; Stacey, 1982; Taub, 1980) including protection from infanticide, provided that the males' estimates of paternity probability are high (van 
Schaik et al., 2004). Thus, females may kill two birds with one stone by mating with multiple males, namely increasing the number of protectors, and decreasing the likelihood that males become attackers.

Female promiscuity, however, requires that females cannot be monopolized by a single male. Therefore, females living in multimale-multifemale groups should attempt to lower male monopolization potential. A variety of female mechanisms may work together in order to break the dominant male's monopoly (i.e. alterations in female sexual behavior and physiology; reviewed in Clarke et al., 2009; see below). Once females are more flexible in terms of mating decisions, they should raise paternity estimates for a few males while at the same time keeping them low but non-zero for many males (sensu van Schaik et al., 2004; see also Soltis, 2002; Soltis \& McElreath, 2001). This 'mixed-mating strategy' enables females to overcome the 'female dilemma', i.e. to balance paternity concentration and dilution (e.g. van Schaik et al., 1999, 2000, 2004).

Given the antagonistic coevolution of reproductive strategies, with males and females following their own interests in order to maximize their reproductive success, the question which sex primarily controls mating and reproductive outcome arises (although coevolutionary races may not lead to stable equilibria; Thornhill \& Gangestad, 2008). Assuming that males predominantly control reproduction, previous investigations mainly focused on male-male competition (e.g. Gowaty, 2004; Parker, 1979). But how successful are females in pursuing their reproductive strategies? To what extent are females able to diminish male monopolization potential and to 'ideally' confuse and dilute paternity, i.e. to manipulate male paternity estimates? Accordingly, how much information do females reveal about their reproductive state? In recent years, researches more frequently addressed questions from the females' perspective in order to illuminate how successful females are in manipulating male mating opportunities and in choosing their mates, given that males might have different interests. However, females, in this respect, are still the lesser-known but by no means the less fascinating sex (e.g. Setchell \& Kappeler, 2003). The variety of female sexual (counter-) strategies and, in particular, the underlying proximate mechanisms, and the interspecific differences may pose a challenge to researchers, but at the same time are crucial in order to understand coevolutionary processes that shape mating systems.

\section{Unpredictable fertility, timing of sexual activity \& cues to fertility}

Female non-human primates exhibit numerous mechanisms to decrease male monopolization potential including extended female sexuality, reproductive synchrony, active mate choice, sneaky copulations, copulation calls, sexual swellings, and an increased number of cycles to conception (e.g. van Schaik et al., 1999, 2004; van Noordwijk \& van 
Schaik, 2000). These 'countertactics' are all based on the assumption that fertility is unpredictable (e.g. Clarke et al., 2009).

In most primates, female mating periods are prolonged, i.e. receptivity (= the female's willingness to accept the male and to permit copulation; Beach 1976) occurs throughout the cycle with a copulatory peak at midcycle, whereas in some species it is limited to the follicular and peri-ovulatory phase (reviewed in Dixson, 1998; Hrdy \& Whitten, 1987; van Schaik et al., 1999). In Old World monkeys, in particular, prolonged mating periods are common and have been attributed to the long follicular phases within ovarian cycles (e.g. van Noordwijk \& van Schaik, 2000; van Schaik et al., 1999). Also, by increasing the number of cycles to conception females may increase the length of their total mating period (e.g. Clarke et al., 2009; Nunn et al., 2001). In addition, numerous non-human primates engage in nonprocreative receptivity (e.g. during pregnancy or lactation; e.g. Dixson, 1998; Hrdy, 1979; Soltis, 2002). This 'extended female sexuality' (Thornhill \& Gangestad, 2008) decreases male monopolization potential, hence enabling females to mate promiscuously and to obtain benefits from multiple males, namely to decrease infanticide by males or to increase male care for future infants (e.g. Borries et al., 1999; Heistermann et al., 2001a; Hrdy, 1979, 2000; Soltis, 2002; van Schaik, 2000b; van Schaik et al., 2004). Although direct male care is rare among primates (Whitten, 1987) protection from infanticide constitutes a form of male infant care (e.g. Borries et al., 1999; Palombit et al., 2000; Paul et al., 2000).

Females may reduce the ability of a single male to monopolize all matings, i.e. decrease male reproductive skew, by clustering their receptive periods in time (e.g. Altmann, 1962; Cowlishaw \& Dunbar, 1991; Emlen \& Oring, 1977; Nunn, 1999a; Ostner et al., 2008a; Ridley, 1986). Reproductive asynchrony, on the contrary, facilitates monopolization by males, decreases female-female competition, promotes indirect mate choice through malemale competition, and increases the chance to conceive (e.g. Ims, 1988; Matsumoto-Oda et al., 2007; Pereira, 1991; Zinner et al., 1994). In seasonally breeding species, reproductive synchrony may simply arise as a consequence of the limited period of mating activity which makes females more likely to become simultaneously receptive (e.g. Cowlishaw and Dunbar 1991; Ridley 1986). Alternatively, but not mutually exclusive, the number of simultaneously sexually active females can be increased by synchronizing ovarian cycles (e.g. French \& Stribely, 1987; Handelmann et al., 1980; McClintock, 1971; McClintock, 1978; Wallis, 1985). However, numerous studies found no evidence for ovarian cycle synchronization (e.g. Monfort et al., 1996; Setchell et al., 2010a; Tobler et al., 2010). Hence, both the question whether or not female mammals (including primates) synchronize their cycles and the methods applied to measure synchrony have been subject to ongoing debate (e.g. Ims, 1990; Pereira, 1991; Schank, 2001a,b,c). 
Another female feature commonly associated with female reproductive strategies is the display of visual, auditory, or olfactory cues to fertility. In this respect, sexual swellings and copulation calls have received most attention as they vary markedly in their appearance among species, with some species lacking these traits (Dixson, 1998; van Noordwijk \& van Schaik, 2000; see below). Coloration and swelling of sex skin are found in many Old World primate species, most of them living in multimale-multifemale groups (Clutton-Brock \& Harvey, 1976; Nunn et al., 2001). Changes in size and coloration of female sexual swellings reflect changes in estrogen and progesterone secretion (i.e. the $E / P$ ratio), with ovulation occurring during the period of maximum swelling size (e.g. Dixson, 1983). Sex skin swellings have evolved at least three times (Cercopithecinae, Colobinae and Hominoidea) in Old World primates (Dixson, 1983; Hrdy \& Whitten, 1987), and several species display an exaggeration of this trait (Nunn, 1999b). It has been argued that sexual swellings and copulation calls function as "graded signals" of ovulation probability (for alternative hypotheses see Hamilton, 1984; Nunn, 1999b; Zinner \& Deschner, 2000; Pagel, 1994; Clutton-Brock \& Harvey 1976; Hrdy \& Whitten, 1987; Burt, 1992; Anderson \& Bielert, 1994; Boesch \& Boesch-Achermann, 2000; reviewed by Thornhill \& Gangestad, 2008) which, by inciting direct male-male competition, enable females to simultaneously concentrate paternity in dominant males, while on the other hand, confusing it amongst others (e.g. Brauch et al., 2007, 2008; Nunn, 1999b; Semple \& McComb, 2000; Semple et al., 2002; Zinner \& Deschner, 2000). In contrast, Thornhill \& Gangestad (2008) argue that generally females should not be selected to signal fertility in their cycles, and that sexual swellings are honest signals of individual female quality and condition (see also Pagel, 1994; Domb \& Pagel, 2001).

Collectively, given the number of possible and presumably unequally costly (see Clarke et al., 2009) alterations in female sexuality, reproductive physiology and morphology, and furthermore, the observed variety across species (e.g. Clarke et al., 2009; Dixson, 1998), a thorough investigation of these traits is crucial as they may mirror the severity of intersexual conflict resulting from antagonistic male and female reproductive strategies (e.g. Clarke et al., 2009; Pradhan \& van Schaik, 2008).

\section{Constraints of reproductive \& life-history traits}

Female reproductive strategies and underlying proximate mechanisms are molded and constrained largely by ecological factors and specific reproductive and life-history traits (e.g. Kappeler \& Pereira, 2003). Thus, careful determination of such features is fundamental when studying any aspect of sexual conflict. In particular, the degree of breeding seasonality of the species in question may considerably shape female reproductive physiology, i.e. patterns of 
ovarian cyclicity which, in turn, are likely to affect female (and male) sexual behavior and mating strategies.

In many primate species, breeding is restricted to a limited period within the year (Brockman \& van Schaik, 2005). Seasonal breeding implies a transition (physiological and behavioral) from a non-reproductive to a reproductive state likely caused by environmental factors (i.e. photoperiod, temperature, rainfall, food abundance; see Brockman \& van Schaik, 2005; Knobil \& Neill, 2006). In strictly seasonally breeding species with very short mating seasons, females exhibit only a few ovarian cycles to conception (e.g. Möhle et al., 2005; Lindburg, 1971; Takahata, 1980), which may limit their opportunities to manipulate male paternity estimates. Apes and Old World monkeys exhibit menstrual cycles, ranging from 2535 days (reviewed by Hrdy \& Whitten, 1987). During the follicular phase, estrogen concentrations gradually increase and peak at midcycle, causing a surge of gonadotropins that stimulates ovulation. After ovulation a corpus luteum is formed and secretes progesterone (luteal phase). If the egg is not fertilized, levels of progesterone fall and menstruation begins. Although in numerous primate species mating is decoupled from hormonal control to some extent (e.g. Dixson, 1998; Wallen, 1990), these cyclic hormonal changes are likely to control female sexual behavior which usually fluctuates during the menstrual cycle (e.g. Nelson, 2005; Dixson, 1998; Baum, 1983; Baum et al. 1977). In line with this, assessment of female reproductive state and physiology allows researchers to relate behavioral or morphological patterns (e.g. sexual swellings) in time to ovulation. Thus, primary determination of hormone profiles throughout and beyond (e.g. pregnancy) ovarian cycles, i.e. an 'endocrine characterization' of female reproductive state, is a crucial first step prior to investigating female sexual behavior and strategies.

Non-invasive methods, i.e. the measurement of urinary or fecal sex steroid metabolites (estrogens, progestogens, and androgens) using Enzyme Immuno Assays (EIA) are useful tools to study wild animals' reproductive endocrinology (e.g. reviewed by Anestis, 2010; Whitten et al., 1998). Thereby, ovarian cycle characteristics (i.e. lengths of cycles and component phases), the timing of ovulation and conception, and hence, also the number of cycles to conception can be well determined even under field conditions (e.g. capuchin monkeys: Carosi et al., 1999; langurs: Ziegler et al., 2000; chimpanzees: Emery \& Whitten, 2003; macaques: O'Neill et al., 2004; Engelhardt et al., 2004; Heistermann et al., 2008; baboons: Higham et al., 2008; callitrichids: Heistermann et al., 1993; gibbons: Barelli et al., 2007). Similarly, steroid hormone profiles during pregnancy may become essential when interpreting post-conception mating (see above), or in addition, may be useful for prenatal infant sex determination or miscarriage detection (e.g. Altmann et al., 2004; Drea, 2011; Dubuc et al., 2009; Ostner et al., 2003). 


\section{Assamese macaques as study species}

Macaques are of particular interest in the context described above because, although sharing the basic patterns of social organization (i.e. multimale-multifemale groups, female philopatry, male dispersal), they exhibit great variation in social relationships, reproduction, and sexual signals (e.g. Dixson, 1998; Thierry, 2007). I studied reproduction, life-history, and mating patterns in a wild population of Assamese macaques (Macaca assamensis, Cercopithecidae, Primates) at Phu Khieo Wildlife Sanctuary, northeastern Thailand. Although very little is known about this species, some findings from a few recent studies raise important questions relating to intersexual conflict, hence making Assamese macaques an interesting model species to investigate female reproductive strategies and the underlying reproductive physiology.

Assamese macaques breed seasonally (Bernstein \& Cooper, 1999; Ostner et al., 2008b) which is likely to account for some degree of reproductive synchrony; however, no data on the timing of conceptions (and, subsequently, births) are yet available. Furthermore, apart from a few details on menstrual cycle characteristics in captive individuals (Wehrenberg et al., 1980), no information about reproductive and life-history parameters (i.e. the number of cycles to conception, gestation length, age at first birth, and interbirth interval) are available for this species. Information on sexual signals is scarce but a few studies suggest that sex skin swellings are not pronounced, with adolescent females exhibiting larger swellings than adult females (Dixson, 1983; Anderson \& Bielert, 1994; Hrdy \& Whitten, 1987). Recent studies on male Assamese macaques indicate a low direct male-male competition for receptive females, reflected in both low alpha male paternity (33\%; Schülke et al., 2010) and low alpha male mating skew which in fact, and despite a clear linear male dominance hierarchy (Ostner et al., 2008b), is the lowest when compared to other macaque species (17.5\%; Ostner et al., 2011). These data indicate that the males' ability to assess female reproductive state is probably poor, hence raising the questions which proximate mechanisms female Assamese macaques exhibit that obscure ovulation from males and lower their monopolization potential, and furthermore, whether females are selective of their mates although mating promiscuously. No study, however, has yet focused on the female perspective in this species, an important gap which this PhD-project aims to fill.

\section{Objectives of this thesis}

Overall, this thesis aims to answer a number of general as well as specific questions about endocrine, morphological, and behavioral correlates of reproductive status, life-history and sexual strategies in wild female Assamese macaques. By combining behavioral and 
hormone data from two consecutive mating seasons, I aim to gain a better understanding of the determinants of mating and reproductive outcome in this species and in primates in general. The different objectives are described in detail as follows:

Chapter 1 is dedicated to determining basic reproductive parameters and life-history traits as a fundamental basis to study female mating strategies. More precisely, I investigate ovarian cycle characteristics, and determine the number of cycles to conception. Also, I establish the degree of seasonality, i.e. the temporal distribution of conceptions and births across the year. Furthermore, I ascertain interbirth intervals and average gestation length by combining hormonal data and birth records. Additionally, I study the information content of sexual swellings, i.e. I investigate whether swellings function as signals of fertility and attractivity in this species.

In Chapter 2, I focus on heterologous sex steroids, i.e. androgens which play a considerable role also in female reproduction but have not been paid much attention in the past. In other words, I expand on the endocrine characterization of female reproductive state (Chapter 1 ) which, for obvious reasons, traditionally involves estrogens and progestogens (i.e. "female hormones"). In detail, I characterize fecal androgen production across the ovarian cycle (i.e. during follicular and luteal phases) and, in particular, throughout pregnancy. Furthermore, I investigate fetal sex effects on maternal fecal androgen concentrations, i.e. I test whether mothers carrying male fetuses exhibit higher fecal androgen concentrations compared to female-carrying females in order to examine whether infant sex can be prenatally determined in Assamese macaques.

In Chapter 3, I investigate the adaptive significance of female sexual behavior. To do so, I combine hormone and behavioral data to ascertain to what extent fertility is obscured in this species, hence reducing female monopolizability by males and decreasing male mating skew. I establish the patterns of female sexual activity, i.e. receptivity and mating frequencies, relative to reproductive state (determined by means of fecal hormone measurements; Chapter 1) to determine to which extent females reveal information about their fertility status. Accordingly, as an indirect measure of male ability to discern female fertile phases, I investigate the distribution of sexual consortships, i.e. male attempts to monopolize fertile females. Furthermore, I determine whether Assamese macaque females exhibit a 'mixed mating strategy', i.e. I investigate whether females balance paternity concentration and dilution.

In Chapter 4, I examine patterns of female reproductive synchrony to test whether female Assamese macaques synchronize their ovarian cycles to thereby potentially decrease male monopolization potential. First, I investigate whether female fertile periods (determined by means of hormone measurements; Chapter 3) are more synchronous or asynchronous than expected by chance. Also, I test whether dyadic spatial proximity affects the distribution 
of fertile phases in order to test the hypothesis that those females who spend more time in close proximity cycle more closely together. Secondly, and irrespective of the occurrence or absence of ovarian cycle synchrony, I propose and test an alternative, non-mutually exclusive hypothesis that females behaviorally synchronize their mating activity. 


\section{CHAPTER 1}

\section{Reproductive and life history parameters of wild female Macaca assamensis}

Fürtbauer, I., Schülke, O., Heistermann, M., \& Ostner, J. (2010). Reproductive and life history parameters of wild female Macaca assamensis. Int. J. Primatol. 31, 501-517.

\section{ABSTRACT}

Information on basic reproductive parameters and life-history traits is crucial for the understanding of primate evolution, ecology, social behavior, and reproductive strategies. Here, we report $4 \mathrm{yr}$ of data on reproductive and life-history traits for wild female Assamese macaques (Macaca assamensis) at Phu Khieo Wildlife Sanctuary, northeastern Thailand. During 2 consecutive reproductive seasons, we investigated reproductive behavior and sexual swelling size in 16 females and collected 1832 fecal samples. Using enzyme immunoassays, we measured fecal estrogen and progesterone metabolites to assess ovarian activity and timing of ovulation and to ascertain conceptions and pregnancies. Timing of reproduction was strictly seasonal (births: April-July, 86\% in April-June, $4 \mathrm{yr}, \mathrm{n}=29$; conceptions: October-February, 65\% in December-January, 2 yr, $n=17)$. Females showed no cyclic ovarian activity outside the mating season and conceived in their first or second cycle (mean: 1.2 cycles to conception, $n=13$ ). Gestation length was on average $164.2 \mathrm{~d}$ (range: 158-170, $n=10)$, and females had their first infant at an age of $5 \mathrm{yr}(\mathrm{n}=4)$. Interbirth intervals were bimodally distributed, with females giving birth on average every 13.9 or 23.2 mo. Shorter interbirth intervals were linked to early parturition within the birth season. Most females displayed subcaudal sexual swellings which, however, did not reliably indicate female reproductive status or fertility. Overall, our results fall within the range of findings reported for other macaque species. These results thus add to the growing body of information available for wild macaques, facilitating comparative studies for a better understanding of interspecific differences in social and reproductive patterns.

\section{KEY WORDS}

Assamese macaques, fecal hormone analysis, gestation length, interbirth interval, seasonality, sexual swellings 


\section{INTRODUCTION}

The diversity and plasticity of primate life-history traits, e.g., age at first birth, gestation length, weaning age, interbirth interval, and underlying reproductive parameters, e.g., number of ovarian cycles to conception, timing of cyclic ovarian activity and reproduction, ovarian cycle characteristics, allow researchers to address questions relating to evolution, ecology, and social behavior (Dixson, 1983; Hrdy \& Whitten, 1987, Kappeler \& Pereira, 2003; Lee \& Kappeler, 2003). In particular, sexual strategies are assumed to be molded by lifehistory (Kappeler et al., 2003). Thus, investigation of female basic reproductive parameters and life-history traits is a crucial first step when studying reproductive strategies used by males and females, and when exploring how they are associated with each other.

Macaques provide ideal conditions for comparative studies concerning the linkage between life-histories and reproductive strategies. All macaque species share basic patterns of social organization: They all live in multimale-multifemale groups and they mate promiscuously, males disperse, whereas females are philopatric (Thierry, 2007). On the other hand, they vary in social and reproductive traits, e.g., the degree of seasonality, with births occurring year-round or being restricted to a few months; social structure, dominance relationships; mating patterns; and the development of sexual signals, e.g., sexual swellings, sex skin coloration, copulation calls, all of which may influence the reproductive strategies of both sexes (Dixson, 1998; van Noordwijk \& van Schaik 2004).

Although the number of studies on macaques steadily increases, some species remain virtually unexplored. Moreover, owing to the difficulties of collecting data from naturally reproducing wild populations, some species are exclusively investigated under captive or free-ranging conditions. However, studies on reproductive and life-history parameters of wild individuals are crucial since it is well known that provisioning can influence reproductive physiology leading, for instance, to an acceleration of puberty and thus earlier age at first reproduction, e.g., Macaca fuscata: Takahata et al. (1998); Papio hamadryas: Zinner et al. (1994), or to a shortening of interbirth intervals (Takahata et al., 1998) compared to the situation found in naturally reproducing populations in the wild. Further, non-natural conditions may blur the degree of reproductive seasonality, e.g., females may cycle and breed throughout the year in captivity, whereas in the wild reproduction of the same species may be restricted to a defined period (Brockman \& van Schaik, 2005).

We investigated female reproductive parameters and life-history traits in a little known species, the Assamese macaque (Macaca assamensis) applying noninvasive hormone analyses. Assamese macaques are sexually dimorphic, breed seasonally (Bernstein \& Cooper, 1999; Ostner et al., 2008b) and, like other macaques, live in multimale-multifemale groups with female philopatry and male dispersal. They are most closely related genetically 
to Tibetan macaques (Hoelzer \& Melnick, 1996) and have been classified as a species with a rather relaxed dominance style (Bernstein \& Cooper, 1999; Cooper \& Bernstein, 2002; Thierry et al., 2000). However, to our knowledge, apart from 1 study on female menstrual cycle patterns in captive Assamese macaques (Wehrenberg et al 1980), no data are currently available on female reproductive parameters and life-history traits in this species. Here, we report for the first time data on breeding seasonality, i.e., timing of conceptions and distribution of births, female age at first birth, number of cycles to conception, gestation length, interbirth interval, and sexual swelling patterns obtained from a naturally reproducing wild Assamese macaque population living in northeastern Thailand.

\section{METHODS}

Study site and subjects

We conducted the study between 2006 and 2009, including collection of detailed behavioral data and fecal samples during 2 complete mating seasons (MS, October to early February) and one nonmating season (NMS, mid February to September), at the Phu Khieo Wildlife Sanctuary (PKWS, 157,300 ha, $16^{\circ} 5^{\prime}-35^{\prime} \mathrm{N}, 101^{\circ} 20^{\prime}-55^{\prime} \mathrm{E}, 300-1300$ masl), northeastern Thailand, which consists of dry evergreen forest with patches of dry dipterocarp forest and bamboo stands (Borries et al., 2002). We collected data from a fully habituated group of Assamese macaques consisting of 53 individuals (12 adult females) during the MS 2007/2008 and 55 individuals (13 adult females) during the MS 2008/2009. In total, we collected fecal samples and data on sexual swellings from 16 females (11 multiparous, 4 primiparous and 1 nulliparous females), 8 individuals being followed in each season. We selected females which, based on their reproductive history, were considered most likely to conceive in the respective MS. One female disappeared around the estimated date of parturition in June 2008, and 1 pregnant female died in March 2009. Demographic data, i.e., births and deaths for all individuals, were available from May 2006 through August 2009.

Fecal sample collection and hormone analyses

We collected and analyzed a total of 1832 fecal samples from 16 focal females. To characterize ovarian cycle patterns and assess timing of conception, we collected on average $4.6 \pm 0.5$ samples/wk from each focal animal during the two MSs. During the NMS we collected 4-7 samples/mo from all focal females, and, after females gave birth in 2008, we continued collection of feces from lactating females $(n=6 ; 1$ sample/wk) until the end of the MS 2008/2009. We collected samples directly after defecation, and placed a 5-15g well 
homogenized portion in a tube labeled with subject ID and date, kept them cold using ice packs during the day, and froze them immediately at $-20^{\circ} \mathrm{C}$ after return to the field station. Samples were stored frozen until being shipped to the endocrine laboratory. We collected samples opportunistically throughout the day; however, we collected the majority of samples (83\%) between 8:00 and 13:00 h.

For hormone analysis, we lyophilized and pulverized fecal samples and extracted an aliquot of $0.05-0.08 \mathrm{~g}$ with $3 \mathrm{ml} 80 \%$ methanol in water by vortexing for 10 min (Heistermann et al., 1995). After centrifugation (3000 rpm, $10 \mathrm{~min}$ ) of the fecal suspension, we decanted the supernatant into a glass tube and stored it at $-20^{\circ} \mathrm{C}$ until assay. We analyzed fecal extracts for concentrations of immunoreactive estrone conjugates (E1C) and 20adihydroprogesterone (20a-OHP) using microtiterplate enzyme immunoassays (EIA), previously shown to reflect accurately female ovarian function in macaques and other primate species (Dubuc et al., 2009; Heistermann et al., 2001b; 1995; Shideler et al., 1993). Details of the EIA procedures have been described previously by Heistermann and Hodges (1995; E1C) and Heistermann et al. (1995, 20a-OHP). Sensitivities of the assays at $90 \%$ binding were $1.0 \mathrm{pg}$ for E1C and $1.5 \mathrm{pg}$ for $20 \mathrm{a}-\mathrm{OHP}$. Intra- and interassay coefficients of variation, calculated from replicate determinations of high- and low-value quality controls were $5.7 \%$ and $6.5 \%$ (high) and $8.8 \%$ and $14.5 \%$ (low) for E1C measurements and $7.5 \%$ and $13.1 \%$ (high) and $9.2 \%$ and $16.7 \%$ (low) for $20 \alpha-O H P$ determinations. All fecal hormone data are expressed as mass per gram of dry feces.

\section{Timing of ovulation and conception and number of cycles to conception}

Since fecal estrogen profiles did generally not show a consistent preovulatory peak (see Results) for unknown reasons, we solely used the fecal progestogen profiles to determine the number of cycles to conception and the presumed day of ovulation and conception. The postovulatory progestogen rise is a reliable indicator that ovulation has indeed occurred, and this is the most widely used approach for determining timing of ovulation in primates (Barelli et al., 2007; Engelhardt et al., 2004; O'Neill et al., 2004; Strier \& Ziegler, 1997). As described in many other studies (e.g. Carosi et al., 1999; Engelhardt et al., 2004; Heistermann et al., 2008), we used the defined rise in fecal progestogen levels above a threshold of the mean \pm 2 SD of 5 preceding baseline values to indicate the onset of the postovulatory (luteal) phase of an ovarian cycle or the onset of gestation (indicated by maintenance of elevated luteal phase progestogen levels for $>4 \mathrm{wk}$ ). Levels below this threshold indicated the follicular phase of the cycle. On the basis of a time lag of about $2 d$ in the excretion of progesterone metabolites in the feces of macaques (Shideler et al., 1993), we considered d -3 relative to the day of the defined progestogen increase $(\mathrm{d} 0$ ) as the most likely day of ovulation. 
Because of the possible variability in the temporal relationship between ovulation and the postovulatory fecal progestogen increase (Shideler et al., 1993), and because daily samples during the periovulatory period were not always available, data on the timing of ovulation/conception can be expected to include an error of 1-2 d.

\section{Sexual swellings}

Assamese macaque females exhibit subcaudal sexual swellings. We visually categorized swelling size on an almost daily basis using 5 categories ranging from 0 to 4 , defined as follows. $0=$ no swelling; 1 = changes in the appearance of subcaudal skin (the skin becoming wrinkled and/or more visible); 2 = slight swelling; 3 = small swelling, 4 = large swelling with lateral bumps (Fig. 1).
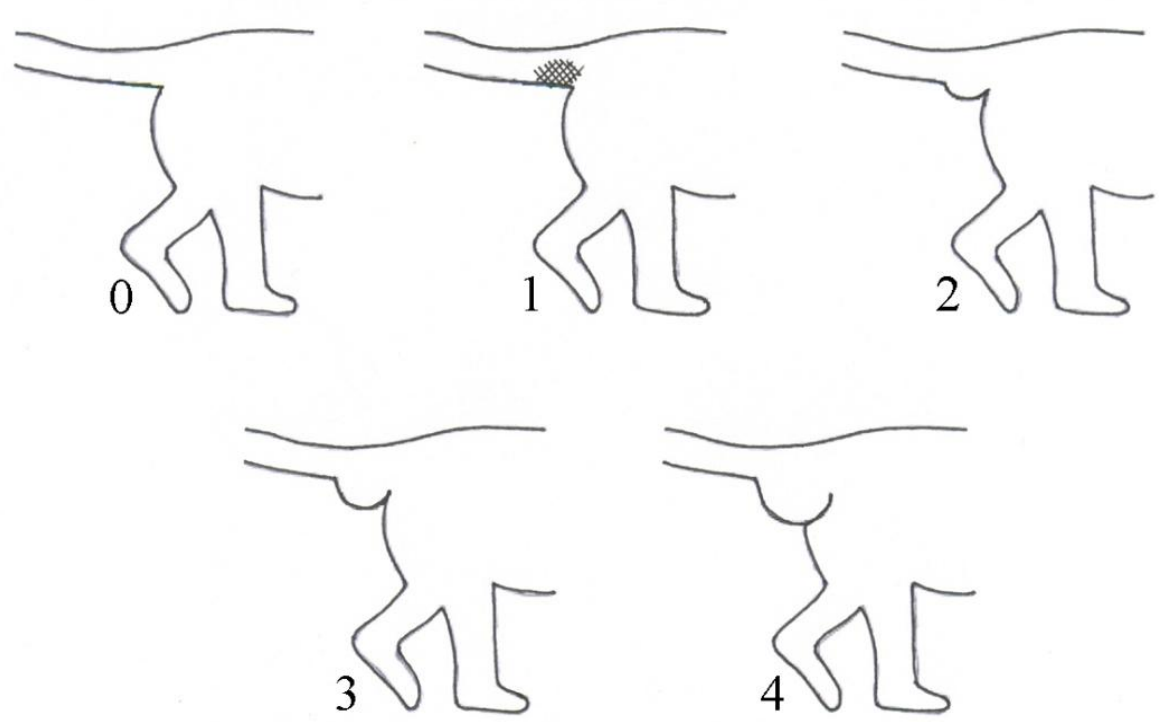

Figure 1: Subcaudal swelling size categories. $0=$ no swelling; $1=$ changes in the appearance of subcaudal skin (visibility; wrinkles); 2 = slight swelling; 3 = small swelling, $4=$ large swelling with lateral bumps.

\section{Life-history traits}

\section{Age at first birth}

We estimated age at first birth for primiparous females retrospectively based on body size comparisons with different age cohorts, which can be clearly distinguished owing to the strong reproductive seasonality. 


\section{Gestation length}

We calculated gestation length in individual females as the interval between the hormonally determined day of conception and the observed day of parturition.

Interbirth interval (IBI) and postpartum amenorrhea (PPA)

We calculated interbirth intervals in days and then converted them to months ( $1 \mathrm{mo}=30.4 \mathrm{~d}$ ) on the basis of observed parturitions. In cases where the exact day of parturition was unknown ( 9 of 24 births), we used the midpoint of the first and the last possible day of parturition (unknown period of max. $12 \mathrm{~d}$ ). We included only IBIs following a surviving offspring (>12 mo). We calculated the duration of PPA for females with 1-yr IBls by counting the days between parturition and next conception. We applied binary logistic regression with SPSS 17.0 to analyze the relationship between the occurrences of 1-yr IBIs and the timing of parturitions within the birth seasons. We used the occurrence of 1-yr IBIs as the dependant, and the number of days between the first day of the first month in which births occur (April 1) and the date of parturition as the independent variable. The 1-sided significance level was set at $p<0.05$.

\section{RESULTS}

\section{Breeding seasonality}

Reproduction in Assamese macaques was highly seasonal. The period when conceptions occurred lasted from October to early February, with the vast majority of conceptions (65\%) being confined to December and January ( $n=17,2$ years; Fig. 2; Table I). A total of 29 infants were born between 2006 and 2009 (Fig. 2). In 2006, 2007 and 2008, all infants were born between April and June, whereas in 20094 of 9 infants were also born in July. Thus, distribution of births within the 4 study years was highly skewed, with an overall of $69 \%$ of births being confined to May and June and $86 \%$ to the 3-mo period of April-June. 


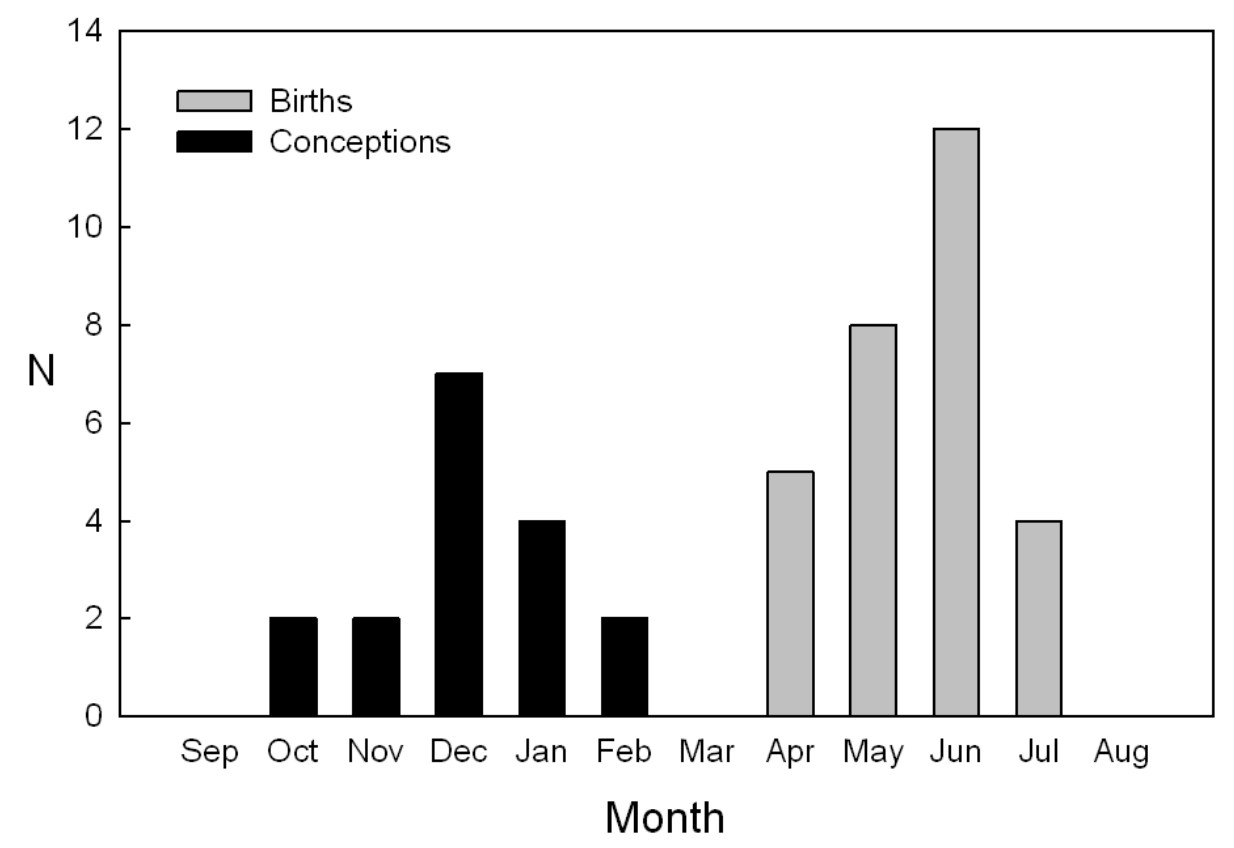

Figure 2: Monthly distribution of conceptions ( $n=17 ; 15$ females; 2 mating seasons) and births ( $n=29,15$ females; 4 birth seasons).

\section{Female reproductive physiology}

Seven out of 8 focal females conceived in the MS 2007/2008, whereas in the MS 2008/2009 all 8 focal females became pregnant. In addition, in 20092 nonfocal females that had also conceived an infant in 2007/2008 gave birth.

Figure 3 shows 2 profiles of fecal E1C and 20a-OHP, covering the month prior to the mating season, i.e., September, the mating season, the period of gestation until parturition, and early lactation in 2 representative females. As can be seen in Fig. 3, and as evident from endocrine profiles in all other focal individuals, females did not show any cyclic ovarian activity prior to the onset of the mating season in October. Conception was clearly visible by a significant rise in fecal progestogen levels that remained elevated (above preconception levels) for the remainder of gestation. After parturition, progestogen levels dropped to baseline concentrations again and remained low during early lactation. In contrast to the progestogen profiles, the patterns of fecal estrogens were less clear. Specifically, a preovulatory estrogen peak was seen in some of the animals (Fig. 3A), but not in others (Fig. 3B). In addition, although estrogen levels, on average, showed a modest increase during gestation, this was not seen in all study females. Based on the hormonal data, ten out of the thirteen conceptions occurred during the first cycle (Fig. $3 \mathrm{~A}$ ), while the remaining 3 females conceived during their second cycle (Fig. 3B). For the 3 females that conceived in the second 
cycle, cycle length, based on the interval between successive fecal progestogen rises, was: 24, 30, and 38 days with an 11-, 15-, and 13-day luteal phase.
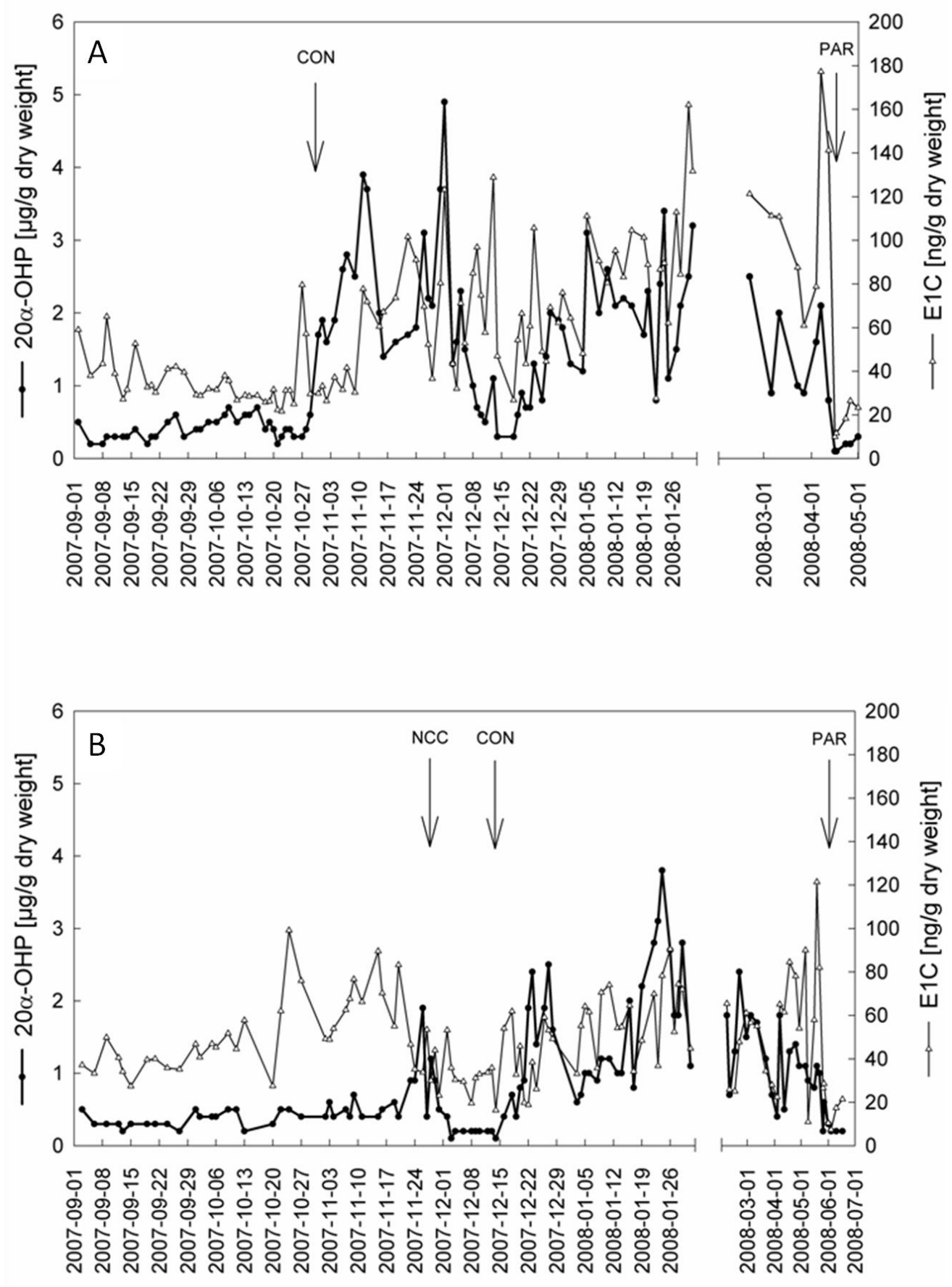

Figure 3: Individual profiles of fecal immunoreactive progestogens (20a-OHP) and estrogens (E1C) in two wild Assamese macaque females covering the period 1 mo before the onset of the mating season, the mating season, gestation, and early lactation, with conception occurring in the first $(A)$ or second $(B)$ ovarian cycle. $C O N=$ conception; PAR = parturition; $\mathrm{NCC}=$ non-conception cycle. Note the adjusted scale after the axis break. 


\section{Gestation length and age at first birth}

For ten females for which the day of conception and the day of parturition were known, length of gestation averaged $164.2 \pm 4.0 \mathrm{~d}$ (median=164.5, $\mathrm{n}=10$, range: $158-170$ ).

Age at first birth was $5 \mathrm{yr}$ for all 4 primiparous females.

\section{Interbirth interval (IBI) \& infant mortality}

For 8 females we were able to calculate $|B|(n=12)$, either on the basis of observed parturitions $(n=10)$ or, for 2 females that disappeared before giving birth, assuming a gestation length of $164 \mathrm{~d}$. The distribution of IBI followed a bimodal pattern with a ca. 1-yr IBI (13.9 $\pm 0.9 \mathrm{mo})$ being seen in 5 of the 12 cases and a 2 -yr IBI $(23.2 \pm 0.9 \mathrm{mo})$ being observed in the remaining 7 cases (Fig. 4). Females that gave birth early in the birth season were more likely to exhibit a 1-yr IBI ( $B=-0.04$, S.E. $=0.03$, df=1, Wald $x^{2}=2.91, p<0.05 ; 1$-sided), and we exclusively observed 1-yr IBIs in multiparous females. PPA for 4 females with a $1-y r$ IBI ranged from 265 to $290 \mathrm{~d}$. Two of the 29 infants (6.9\%) born between 2006 and 2009 died at an age of 4 and $10 \mathrm{mo}$, respectively, and therefore, we did not include them in the IBI calculations.

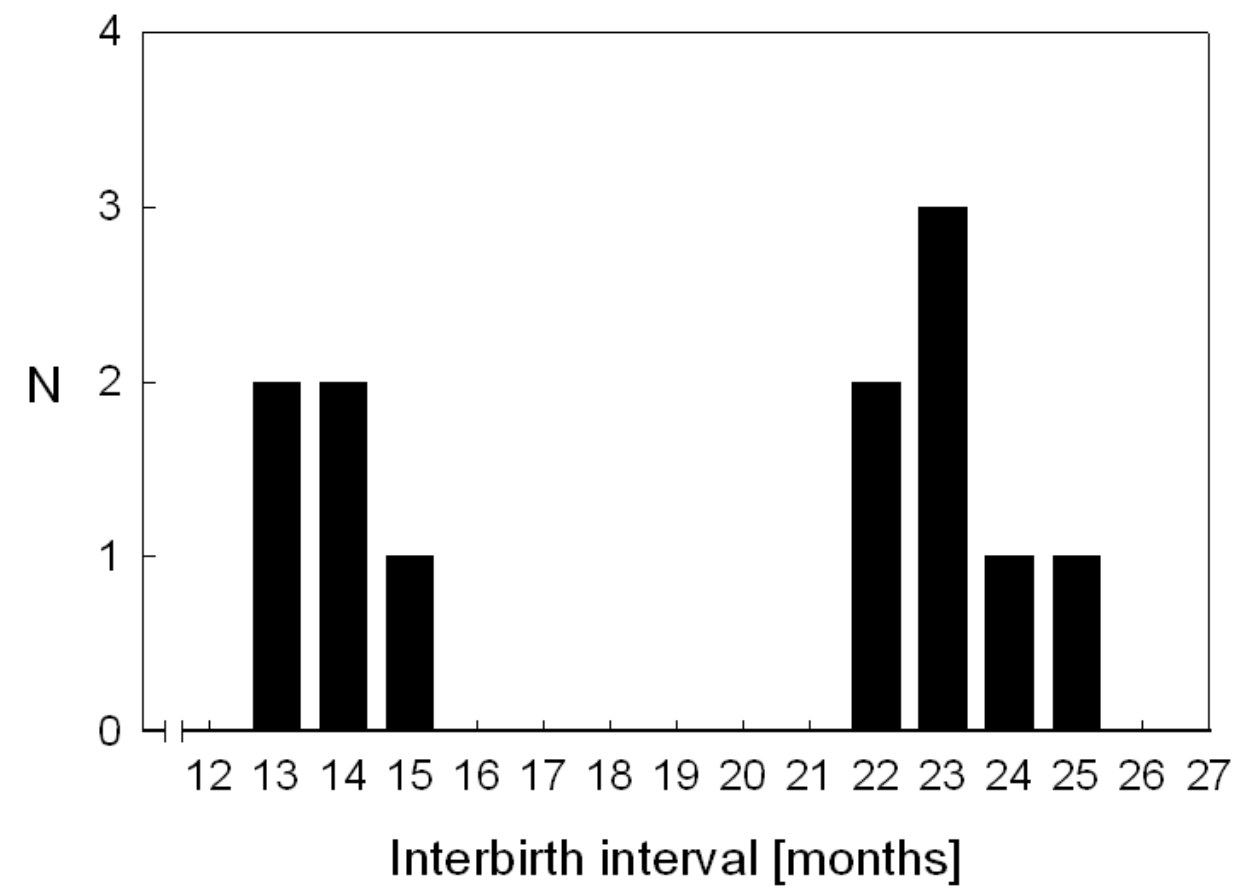

Figure 4: Distribution of interbirth intervals in wild Assamese macaques ( $n=12,8$ females). 
Table 1: Reproductive and life history parameters of wild female Assamese macaques.

\begin{tabular}{|c|c|c|c|c|c|c|c|}
\hline Parameter & & Mean & SD & Median & Range & $\mathbf{n}$ & Unit \\
\hline $\begin{array}{l}\text { Gestation } \\
\text { length }\end{array}$ & & 164.2 & 4.0 & 164.5 & $158-170$ & 10 & Days \\
\hline $\begin{array}{l}\text { Interbirth } \\
\text { interval }^{\mathrm{a}}\end{array}$ & & - & - & 22.2 & $12.6-24.7$ & 12 & Months \\
\hline$\sim 1 \mathrm{yr}$ & & 13.9 & 0.9 & 14.2 & $12.6-14.9$ & 5 & Months \\
\hline$\sim 2 \mathrm{yr}$ & & 23.2 & 0.9 & 23.2 & $22.1-24.7$ & 7 & Months \\
\hline $\begin{array}{l}\text { Cycles to } \\
\text { conception }\end{array}$ & & 1.2 & 0.4 & 1 & $1-2$ & 13 & - \\
\hline $\begin{array}{l}\text { Timing of } \\
\text { conceptions }\end{array}$ & $\begin{array}{l}67 \% \text { in } \\
\text { Dec/Jan }\end{array}$ & - & - & - & Oct-Feb & 17 & - \\
\hline Age at first birth & & 5 & - & 5 & - & 4 & Years \\
\hline Timing of births & $\begin{array}{l}69 \% \text { in } \\
\text { May/Jun }\end{array}$ & 3 & - & 3 & $\begin{array}{l}\text { 2-4 (Apr- } \\
\text { Jul) }\end{array}$ & $4^{b}$ & Months \\
\hline
\end{tabular}

${ }^{\text {a }}$ IBI follows bimodal pattern

${ }^{b} 4$ birth seasons (2006-2009; 29 births)

\section{Sexual swellings}

Of the 16 focal females, the majority $(n=13)$ displayed a sexual swelling but females differed in maximum swelling size attained (Fig. 5), whereas 3 (cyclic) females showed no swelling around ovulation (Fig. 5A). Maximum swelling size for multiparous females $(n=11)$ ranged from 1 to 3 , i.e., multiparous females never reached category 4. Maximum swelling size for primiparous females $(n=4)$ was 4 in 3 cases, and 3 in 1 case. The only nulliparous (acyclic) female in this study attained swelling size category 2 . We could not detect any cyclic pattern in sexual swelling size and swellings did not deflate after conception (Fig. 5B, C). The timing of maximum swelling size attained varied among females, i.e., some females reached their maximum swelling before ( $n=7$; Fig. $5 B$ ) and some ( $n=5$; Fig. $5 C$ ) females after conception. 

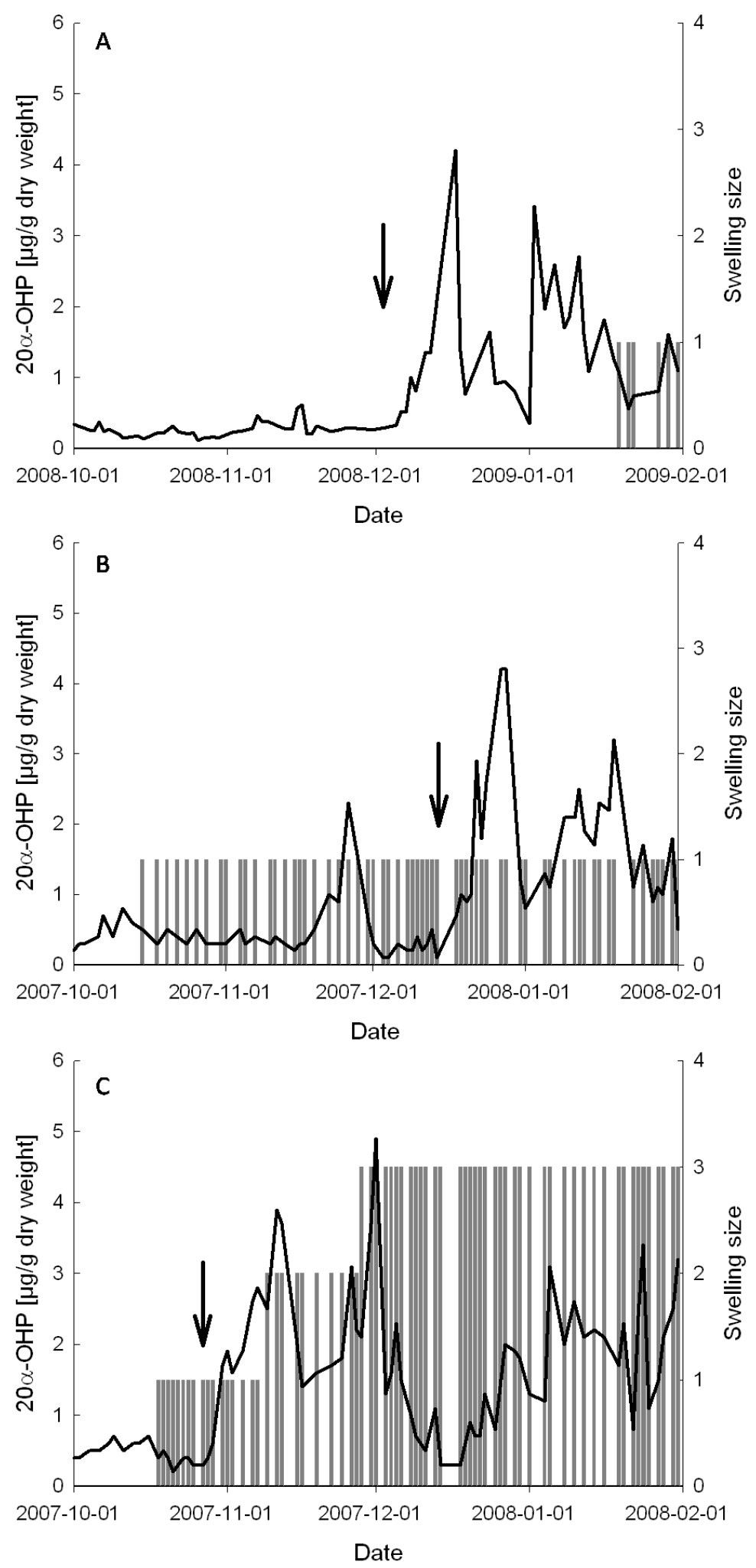

Figure 5: Sexual swelling size (gray bars) in relation to fecal 20a-OHP levels (solid line) and the timing of conception (arrow) in 3 wild Assamese macaque females. The figure illustrates the large variability in swelling patterns between females and the absence of a defined relationship between swelling size and female reproductive status. 


\section{DISCUSSION}

We here describe for the first time female reproductive parameters and life-history traits in wild female Assamese macaques. We present data on reproductive seasonality, basic female reproductive physiology, gestation length, age at first birth, interbirth interval, and sexual swellings, derived from a naturally reproducing wild population. For most parameters, the values found for Assamese macaques fall into the range reported for other macaque species. The distribution of interbirth intervals and the large interindividual variability in swelling patterns, however, appear to deserve further attention.

\section{Reproductive seasonality}

Seasonal timing of reproduction among macaques covers the entire range from year round breeding, e.g., in pigtail macaques (Macaca nemestrina), to extreme seasonal reproduction in the Japanese macaque (Macaca fuscata; range across macaques: 46-365 d; Nunn, 1999a). Seasonal breeding implies physiological and behavioral transition from a nonreproductive to a reproductive state, i.e., females may show a change in reproductive cyclicity (Walker et al., 1983), likely caused by environmental factors (photoperiod, temperature, rainfall, food abundance; Brockman \& van Schaik, 2005).

In our study $65 \%$ of conceptions were confined to December and January and $86 \%$ of births occurred within the 3-mo period of April-June, indicating a strict reproductive seasonality (sensu van Schaik et al., 1999) in Assamese macaques. Similar to Japanese (Hill, 1997) and Barbary macaques (Ménard \& Vallet, 1996), Assamese macaques at Phu Khieo Wildlife Sanctuary live in a markedly seasonal habitat with seasonal changes in rainfall (Grassman et al., 2005) and food availability (Koenig et al., 2004, Schülke et al., unpublished). However, a fine grained analysis of potential triggers for the timing of reproduction is pending.

\section{Female reproductive physiology}

As in previous studies on various primate taxa (e.g. Barelli et al., 2007; Carosi et al., 1999; Dubuc et al., 2009; Engelhardt et al., 2004; Heistermann et al., 2008; Higham et al., 2009; Ziegler et al., 2000), measurement of fecal progestogen excretion proved to be a reliable tool for the assessment of female reproductive status, including the timing of ovulation and conception in Assamese macaques. By contrast, the measurement of fecal estrogens in Assamese macaques appears to be less useful in assessing female ovarian function since estrogen profiles often lacked a clear preovulatory peak and showed no marked elevation in levels during gestation. The reason why our results for Assamese macaques differ in this 
respect from those reported for other macaque species (e.g. Engelhardt et al., 2004; Dubuc et al., 2009; Brauch et al., 2007; Fujita et al., 2001) is not clear. Given that it is unlikely that Assamese macaques differ in their mechanisms of hormonal regulation of the ovarian cycle and pregnancy from other macaques, our finding of less informative fecal estrogen profiles is likely related to species differences in estrogen metabolism and pattern of excretion or assay methodology. In this respect there are 4 nonmutually exclusive potential explanations. First, in Assamese macaques estrogens may be predominantly excreted into the urine rather than the feces, rendering fecal measurements less informative. Second, measurement of other estrogen metabolites than the one measured here might be more adequate for monitoring estrogen excretion in this species. However, analysis of estradiol and total estrogens in a subset of cycles did not show better profiles (data not shown). Third, the E1C antibody used here might have potentially cross-reacted with fecal compounds unrelated to estrogen secretory dynamics, thereby confounding an existing pattern. Last but not least, given the short-term nature of the preovulatory estrogen peak, it cannot be excluded that in some females the estrogen peak was just missed because daily samples were often not available during the peri-ovulatory period. A study on estrogen metabolism and excretion, including HPLC analysis to help clarify 1 ) the identity of the major fecal estrogen metabolites excreted in female Assamese macaques and 2) the potential for co-measurement of nonestrogenic compounds by any given estrogen assay used would be a useful step towards resolving this issue in the future.

Consistent with the strong reproductive seasonality, females showed a typical hormonal pattern, with absence of cyclic ovarian activity during the nonmating season ("flat liner") and resumption of ovarian cycles during the mating season, a phenomenon also seen in other primate species with a highly seasonal reproduction, e.g., Macaca sylvanus: Möhle et al. (2005); Semnopithecus entellus: Ziegler et al. (2000). The lack of ovulatory cycles during the nonmating season was seen irrespective of whether females were nursing an infant or not. This further underscores the impact of the observed seasonality on female reproductive physiology in this species.

Like other seasonally breeding macaque species, e.g., Macaca sylvanus: Küster \& Paul (1984); Möhle et al. (2005); Macaca mulatta: Lindburg (1971); Macaca fuscata: Takahata (1980), Assamese macaque females mostly conceived during the first cycle of the mating season. Hence, ovarian cycle characteristics, i.e. the length of the ovarian cycle (and its component phases), could only be determined for the few females which conceived in their second cycle, and was 24, 30, and 38 days (with 11-, 15-, and 13-d luteal phases). These data fall well into the range of observed cycle lengths in macaques (26.4-38.0 d; Thierry, 2007) and compare well with cycle length data of $32 \pm 2 \mathrm{~d}$ (divided into a $16 \pm 1 \mathrm{~d}$ 
follicular phase and a $16 \pm 1$ d luteal phase) reported by Wehrenberg et al. (1980) for captive animals based on blood hormone analysis.

Gestation among macaques is relatively constant and ranges from 160 to $182 \mathrm{~d}$ (Kappeler \& Pereira, 2003), and female age at first birth in macaques, based on captive and wild data, ranges from 3.5 to $6.5 \mathrm{yr}$ (Melnick \& Pearl, 1987). An average gestation length of $164 \mathrm{~d}$ and an age of first reproduction of $5 \mathrm{yr}$, as found for Assamese macaques here, fit well into the reported ranges.

\section{Interbirth interval (IBI)}

The IBls in Assamese macaques are bimodally distributed, with females giving birth on average 14 mo or 23 mo after the previous birth, i.e., the next year or after $2 \mathrm{yr}$ (range across macaques: 12-24 mo; Kappeler \& Pereira, 2003). The IBI is made up of 3 components: gestation length $(G L)$, postpartum amenorrhea (PPA), and the duration of ovarian cycling to conception (DC). Because GL in any primate species is relatively invariable (Dixson, 1998), differences in IBI within a species as reported here for Assamese macaques can be caused only by differences in the duration of PPA and/or DC (provided that under natural conditions a female would inevitably conceive). Most Assamese macaque females conceived in their first cycle, i.e., DC is minimized. PPA for 4 females with a 1-yr IBI ranged from 265 to $290 \mathrm{~d}$ (birth until next conception), and thus was relatively constant. This indicates that the time that a female needs to resume cycling after giving birth is restricted due to inhibitory effects of lactation (McNeilly, 2001) and maternal body condition. Concerning the latter, studies on several primate taxa have shown that females need to attain a critical body weight before they can resume ovarian cycling, e.g., Papio anubis: Bercovitch (1987); Propithecus verrauxi verrauxi: Lewis \& Kappeler (2005); Macaca nemestrina: Maninger et al. (2000); Semnopithecus entellus: Ziegler et al. (2000); Homo sapiens: Frisch \& McArthur (1974). In line with this, nutritional conditions appear to affect the length of the IBI (Borries et al., 2001; Takahata et al., 1998). Similarly, it has been shown that infants need to reach a minimum body mass threshold for weaning (Lee, 1996; 1999; Lee et al., 1991). Accordingly, the timing of parturition within the birth season may play an important role, simply because females that give birth early in the birth season have more time to reach the conditions required to resume cyclic ovarian activity in the following mating season. Females should therefore be more likely to have an infant in the consecutive year, and thus a short IBI, when they give birth early in the birth season. Our results support this hypothesis because the occurrence of 1-yr IBIs was significantly linked to early parturition within the birth season.

In addition, owing to lactational constraints on ovarian activity, the loss of an infant may shorten the length of the IBI, e.g., Papio cynocephalus: Altmann et al. (1977); 
Trachypithecus leucocephalus: Jin et al. (2009); Macaca maurus: Okamoto et al. (2000); Mandrillus sphinx: Setchell et al. (2002); Macaca fuscata: Tanaka et al. (1970); Macaca thibetana: Zhao \& Deng (1988); Papio hamadryas: Zinner et al. (1994). This effect may be stronger in nonseasonal species than in strictly seasonal species, in which the timing of infant loss may be crucial: if the infant dies late in the mating season or even after the end of the mating season, a female may not be able to resume cycling. This may lead to infanticide only being a favorable strategy for males during a specific time window. In this study, infant mortality was low (6.9\%), and only 1 of 61 -yr IBls was preceded by the loss of a 4 -mo-old infant at the beginning of the mating season for an unknown reason. Another infant died at an age of 10 mo during the nonmating season, and this did not result in a shorter IBI.

Although the loss of an infant may shorten the length of the $\mid \mathrm{BI}$, depending on the timing of the loss, it does not appear to be the ultimate cause for the occurrence of 1-yr IBIs in female Assamese macaques. This and the linkage between 1-yr IBIs and the timing of parturition within the birth season, in turn, suggest that the IBIs are, to some extent, influenced by physiological constraints that may be partly linked to the strong reproductive seasonality. However, long-term data are needed to investigate the potential impact of age, rank, and ecological factors on the distribution of 1- and 2-yr IBIs in this species (e.g. Borries et al., 2001; Robbins et al., 2007; Setchell et al., 2002).

\section{Sexual swellings}

Coloration and swelling of sex skin during the menstrual cycle are found in many Old World primate species, most of them living in multimale-multifemale groups (Clutton-Brock \& Harvey, 1976; Nunn et al., 2001). Changes in size and coloration of female sex skin swellings may reflect changes in estrogen and progesterone secretion, i.e., the E/P ratio, with ovulation usually occurring during the period of maximum swelling size (Brauch et al., 2007; Deschner et al., 2003; Higham et al., 2008). Sex skin swellings have evolved at least 3 times independently in Old World primates (Dixson, 1983; Hrdy \& Whitten, 1987), and several species display an exaggeration of this trait (Nunn, 1999b). Macaque species exhibit pronounced variation in size and coloration of sex skin swellings. Large and pinkish swellings involving skin in the subcaudal, circumanal, paracallosal, vulval, and clitoral areas occur in species of the silenus-sylvanus group, whereas members of the fascicularis, and the sinicaarctoides groups show reduced swellings (Dixon, 1983; 1998). Interestingly, in some macaque species adolescent females exhibit larger swellings than adult females (Anderson \& Bielert, 1994; Dixson, 1983), and swellings or sex skin color changes during early pregnancy (post-conception swellings) are common, e.g., Macaca fascicularis: Engelhardt et al. (2007); Macaca sylvanus: Möhle et al. (2005); reviewed in Hrdy \& Whitten (1987). Not 
only the appearance of sexual swellings but also their functional significance varies among macaque species, i.e., swellings may convey reliable information on female reproductive state, e.g., Macaca sylvanus: Brauch et al. (2007), or, in contrast, may have limited, e.g., Macaca fascicularis: Engelhardt et al. (2005) or no apparent signaling content, e.g., Macaca thibetana: Li et al. (2005).

As predicted for a seasonal species (Dixson, 1998; cf. van Schaik, 1999), Assamese macaque females did not exhibit prominent sexual swellings. We found remarkable interindividual differences in swelling patterns which mostly concur with van Noordwijk's (1985) findings in female long-tail macaques. The maximum swelling size attained varied among females, and some females did not show swellings at all, despite the fact that they were conceiving and thus reproductively normal. Young females showed swellings of larger maximum size, and may have a swelling a year before their first conception (Fürtbauer, pers. obs.). As we found neither apparent cyclic size changes nor a link between swelling patterns and hormonal profiles/timing of conceptions, the observed swelling characteristics suggest that, in contrast to macaque species with a pronounced swelling, the subcaudal swellings found in female Assamese macaques do not reliably indicate female reproductive status and fertility, and may simply be a relic of an ancestral trait. It may be possible, however, that the method used in this study - visual judgment instead of video imaging - did not pick up on very subtle changes in sexual swelling patterns.

\section{Conclusions and implications for female reproductive strategies}

In summary, the reproductive parameters and life-history traits reported in the present study for female Assamese macaques mostly resemble findings for other macaque species. Although this is not surprising, the information generated here is useful in helping to complete the data base on reproductive and life-history traits in wild macaques and thus facilitating species comparisons. More importantly, however, knowledge in this area will also be crucial for future studies investigating the reproductive biology of Assamese macaques. In this respect, the finding of a strong reproductive seasonality with a high degree of clustering in timing of conceptions, and thus suggesting female reproductive synchrony, is likely to influence strongly male and female reproductive strategies and their significance on reproductive success. Because asynchrony within estrus synchrony may occur (Pereira, 1991), however, a thorough analysis of the temporal distribution of fertile phases along with male and female sexual activity is needed to investigate female reproductive strategies. 


\section{ACKNOWLEDGMENTS}

We thank the National Research Council of Thailand (NRCT) and the Department of National Parks, Wildlife and Plant Conservation (DNP) for permission (No. 0004.3/3618) to conduct this study. We thank J. Prabnasuk, K. Nitaya and K. Kreetiyutanont (Phu Khieo Wildlife Sanctuary) for their cooperation and permission to carry out this study. We thank A. Koenig and C. Borries (Stony Brook University), who developed the field site at Huai Mai Sot Yai, for their support. We thank Andrea Heistermann and Jutta Hagedorn for technical assistance in hormone analysis, and M. Swagemakers, D. Bootros, T. Kilawit, A. Chunchaen and P. Saisawatdikul, S. Jomhlotwong, S. Rogahn and W. Nuagchiyos for their help in the field. We thank J. Setchell and 2 anonymous referees for their valuable comments on the manuscript. We also thank N. Bhumpakphan, W. Eiadthong (Kasetsart University) and W. Brockelman (Mahidol University) for their support and cooperation. The project was supported with funds from the National Geographic Society, Max-Planck Society and the German Initiative of Excellence. 



\section{CHAPTER 2}

\section{Fecal androgen excretion and fetal sex effects during gestation in wild Assamese macaques (Macaca assamensis)}

Fürtbauer, I., Heistermann, M., Schülke, O., \& Ostner, J. (submitted) Fecal androgen excretion and fetal sex effects during gestation in wild Assamese macaques (Macaca assamensis). Am. J. Phys. Anthropol.

\section{ABSTRACT}

In placental mammals, pregnancy usually is associated with an increase in maternal androgens which may significantly impact fetal growth and differentiation, and affect postnatal development and behavior. Owing to their slow life histories and challenging social conditions, determination of maternal androgens and potential interference effects of fetal androgen production are of particular interest in wild primates. However, androgen production has been rarely investigated in wild female primates, and studies on maternal androgens during gestation in particular often do not span the entire pregnancy. Here, we characterize fecal androgen production throughout gestation in wild Assamese macaques (Macaca assamensis) using non-invasive hormone analysis and, furthermore, examine fetal sex effects on maternal androgen excretion. A total of 207 fecal samples were analyzed from seven females for concentrations of immunoreactive epiandrosterone (iEA). Fecal iEA concentrations, as predicted based on cercopithecine blood-serum patterns, increased during early gestation and were significantly higher during the first trimester compared to preconception concentrations and those recorded during later stages of gestation. Further, during the third trimester, male-carrying mothers showed significantly higher $\mathrm{iEA}$ concentrations compared to female-carrying mothers. This first characterization of fecal androgen excretion during gestation in Assamese macaques indicates both a maternal and fetal effect on androgen production. Although our sample size is small, our results nevertheless provide the basis for assessing potential influences of maternal androgens on postnatal offspring development and behavior.

\section{KEYWORDS}

fetal effects, maternal androgens, non-invasive hormone analysis, pregnancy 


\section{INTRODUCTION}

In placental mammals, pregnancy is associated with a marked rise in estrogen and progestogen secretion as a result of ovarian and placental function to establish and maintain a healthy pregnancy (Knobil \& Neill, 2006). In a number of species, however, pregnancy is also characterized by a distinct increase in maternal androgen production in the early stages of gestation (Callithrix jacchus: Chambers \& Hearn, 1979; Rattus sp.: Gibori \& Sridaran, 1981; Legrand et al., 1984; Papio cycnocephalus: Castracane \& Goldzieher, 1983; Macaca radiata: Rao \& Kotagi, 1983; Papio anubis: Hodges et al., 1984; Macaca mulatta: Ellinwood et al, 1989; Homo sapiens: Castracane et al., 1998; Callithrix geoffroyi: French et al., 2010; Smith et al., 2010), which is likely to be under the regulation of chorionic gonadotropin (GC) (e.g. Castracane \& Goldzieher, 1983). Since maternal androgens have been shown to have a number of organizational and developmental effects on the embryo and the fetus (e.g. sexual differentiation), and are known to influence postnatal processes like growth, metabolism, and behavior (e.g. Bruns et al., 2004; Goy et al., 1988; Phoenix et al., 1959; Roberts et al., 2009; Smith et al., 2010; Thornton et al., 2009; Tomaszycki et al., 2001; Wallen, 1996, 2005; Wallen \& Hassett, 2009; Wolf et al., 2002), there is increasing interest in characterizing maternal androgen production as a basis for studies on its modifying actions on the morphological, physiological and behavioral development of offspring.

Primates, in particular, have slow life-histories and live in complex societies (e.g. Campbell et al., 2007; van Noordwijk \& van Schaik, 2000), and hence, both prenatal physiological and postnatal social factors are likely to shape the development and behavior of individuals. For example, involving administration of androgens to the pregnant female, a large number of studies on sex differences in non-human primates (mainly rhesus macaques) have shown that both the maternal androgenic environment as well as specific social contexts are crucial for the expression of certain sex specific juvenile and adult behaviors (Goy et al., 1988; Wallen, 1996, 2005; Wallen \& Hassett, 2009).

Furthermore, fetal testes secrete androgens as part of male sexual differentiation which, in turn, may affect maternal androgen concentrations. Hence, in a number of mammal species (including primates) females carrying male fetuses show higher androgen concentrations at certain stages of gestation compared to females carrying female fetuses (e.g. baboons: Altmann et al., 2004; elephants: Duer et al., 2002; humans: Meulenberg \& Hofman, 1991; lemurs: Drea, 2011); however, other studies did not find this relationship (e.g. French et al., 2010; Glass \& Klein, 1981; Steler et al., 2002; Troisi et al, 2003). Thus, maternal androgens can be derived solely from maternal sources or reflect both maternal plus fetal contributions. 
Characterizing the pattern of maternal androgen production during gestation, under consideration of potential blurring fetal effects, provides a basis for investigating the potential impact of androgens on infant development and behavior, and to disentangle the potential influence of underlying prenatal physiological and postnatal social factors on these traits. Field endocrinology techniques based on non-invasively collected samples (e.g. feces or urine) allow studying such endocrine processes in wild-living primates (e.g. Wasser et al., 2000; Whitten et al., 1998). However, whereas the application of such techniques in females is widespread with respect to the measurement of estrogens and progestogens as part of studies characterizing female reproductive function in different contexts (for review see Anestis, 2010), the assessment of female androgen output in wild primate populations is limited (Altmann et al., 2004; Beehner et al., 2005; von Engelhardt et al., 2000; French et al., 2010; Ostner et al., 2003; Smith et al., 2010). Furthermore, to our knowledge, no study has yet investigated patterns of fecal androgen excretion throughout the entire pregnancy, although knowledge on the androgen dynamics throughout gestation would be an important basis for any study interested in the potential role of androgens in pre- and postnatal differentiation and development.

In order to extend the database on maternal androgen profiles in primate pregnancy and to investigate whether fetal sex affects maternal androgen concentrations also in a macaque species, here we (1) characterize fecal androgen excretion from conception to birth in wild Assamese macaques (Macaca assamensis) and (2) investigate potential fetal sex effects on maternal androgen concentrations. The application of non-invasive fecal hormone analysis in this species has been recently proven useful to study the reproductive biology and endocrine-behavior interactions in both males and females (Fürtbauer et al., 2010, submitted; Ostner et al., 2008, 2011). Previous studies on cercopithecines investigating serum androgen concentrations have shown that androgens vary significantly during pregnancy, with a distinct increase during the first trimester, followed by a decline to pre-conception concentrations around day 50 (Macaca radiata: Rao \& Kotagi, 1983; Papio cynocephalus: Castracane \& Goldzieher, 1983; Papio anubis: Hodges et al., 1984). Based on this knowledge on circulating androgen patterns during gestation in closely related species, we predict that in Assamese macaques androgen production significantly increases during early gestation, and declines back to pre-conception concentrations towards the end of the first trimester (e.g. around weeks 7-8). Further, we test the hypothesis that infant sex predicts maternal fecal androgen concentrations, and predict higher fecal androgen concentrations in females carrying male fetuses compared to those carrying female fetuses. 


\section{METHODS}

\section{Study site and subjects}

Seven wild female Assamese macaques (6 multiparous and 1 primiparous females; Table 1) living in a group consisting of 53 individuals provided samples for this study. The animals were living at the Phu Khieo Wildlife Sanctuary (PKWS, 157,300 ha, 165'-35'N, 101 ${ }^{\circ} 20^{\prime}-$ 55'E, 300-1,300 m a.s.I.), north-eastern Thailand, which consists of dry evergreen forest with patches of dry dipterocarp forest and bamboo stands (Borries et al., 2002).

\section{Fecal sample collection and hormone analysis}

Between October 2007 (start of the mating season) and June 2008 (end of the birth season) fecal samples were collected from the seven study females in order to investigate female reproductive and life-history parameters and to hormonally assess conceptions and characterize pregnancies by means of progestogen and estrogen measurements, the data of which have previously been published (Fürtbauer et al., 2010). Frequencies of sample collection varied according to season, with weekly samples being available for all seven study females throughout the entire pregnancy, and more frequent samples during the mating season, i.e. around conceptions. Overall, a total of 207 fecal samples were available and used in the present study to investigate maternal androgen excretion as a function of gestational stage, including the weekly pregnancy samples (mean $\pm s d: 21.4 \pm 0.8$ samples per female; 23.4 weeks of gestation; Fürtbauer et al., 2010) and samples from the late follicular phase (i.e. days -7 to conception; mean \pm sd: $5.4 \pm 1.4$ samples per female) and luteal phase to determine non-pregnant baseline androgen concentrations. Because Assamese macaques mostly conceive during their first ovarian cycle within the mating season (Fürtbauer et al., 2010), luteal phase samples were only available from 3 females (18 samples; 6 each).

Samples not contaminated with urine were collected directly after defecation into a plastic container and were frozen at $-20^{\circ} \mathrm{C}$ within $4-5$ hours after collection upon return to the field station (Fürtbauer et al., 2010). Prior to analysis, fecal samples were lyophilized and pulverized and an aliquot representing $0.05-0.08 \mathrm{~g}$ dry fecal powder was extracted with $3 \mathrm{ml}$ $80 \%$ methanol in water by vortexing for $15 \mathrm{~min}$ (Heistermann et al., 1995). Following centrifugation (3000 rpm, $5 \mathrm{~min}$ ) of the fecal suspension, the supernatant was decanted into a glass tube and stored at $-20^{\circ} \mathrm{C}$ until hormone measurement.

Samples were analyzed for immunoreactive epiandrosterone (iEA), a major metabolite of testosterone in macaque feces (Möhle et al., 2002), and recently shown to provide reliable information on androgen production in wild male long-tailed and Assamese 
macaques (Girard-Buttoz et al., 2009; Ostner et.al, 2011). The iEA measurement was carried out by a microtiterplate enzyme immunoassay (Palme \& Möstl, 1994) as described in detail by Girard-Buttoz et al. (2009). The assay used an antibody raised in a rabbit against $5 \alpha-$ androstane-3a-ol-17-one-HS:BSA, 5a-androstane-3,17-dione-thioether-DADOO-Biotin was used as label and epiandrosterone as standard (Palme \& Möstl, 1994). Sensitivity of the assay at $90 \%$ binding was $1.5 \mathrm{pg}$ and intra- and inter-assay coefficients of variation calculated from replicate determinations of high- and low-value quality controls were $7.5 \%$ and $9.3 \%$ (high) and $8.6 \%$ and $14.5 \%$ (low), respectively. All hormone values are expressed as mean $\pm S E M \mu g / g$ fecal dry weight.

\section{Data analyses}

All analyses were conducted in R (R Development Core Team, 2008). In order to investigate differences in fecal androgen concentrations throughout pregnancy, we divided the 164 days gestation period (Fürtbauer et al., 2010) into trimesters of similar length (i.e. 8 weeks each). iEA concentrations across pre-conception stages and trimesters were tested for an overall statistical difference using a Friedman test based on individual means during trimesters. Post-hoc analyses were carried out using exact Wilcoxon signed ranks tests. Further, exact Wilcoxon signed ranks tests were used to investigate the increase in fecal iEA concentrations at the onset of the first trimester and the decrease around the transition from the first to the second trimester, respectively. To determine whether maternal fecal androgen concentrations are higher in male-carrying mothers compared to female-carrying mothers during trimesters of pregnancy we used one-sided Student's t-tests assuming unequal variances. For all tests, the significance level was set at $p<0.05$.

\section{RESULTS}

\section{Fecal androgen patterns throughout pregnancy}

Pregnancy was associated with clear and significant changes in maternal fecal androgen production (Fig. 1). iEA concentrations were low in the week prior to (mean \pm SEM: $1.8 \pm 0.5$ $\mu \mathrm{g} / \mathrm{g}$ ) and following conception (mean \pm SEM: $1.7 \pm 0.6 \mu \mathrm{g} / \mathrm{g})$ but rose significantly $\left(\mathrm{T}^{+}=21\right.$, $\mathrm{p}=0.031, \mathrm{n}=6$ ) from the first to the second week of gestation (mean \pm SEM: $3.3 \pm 1.0 \mu \mathrm{g} / \mathrm{g}$ ). Elevated concentrations were maintained until week 7 , and iEA concentrations returned to pre-pregnancy concentrations between weeks 7-9, i.e. towards the beginning of the second trimester $\left(T^{+}=21, p=0.031, n=6\right)$. iEA concentrations were low during luteal phases 
(mean \pm SEM: $1.0 \pm 0.1 \mu \mathrm{g} / \mathrm{g} ; \mathrm{n}=3$ females; the remaining 4 females conceived during their first cycle; see Table 1). Conceptions occurred between October $28^{\text {th }}$ and January $6^{\text {th }}$.

Fecal androgen concentrations varied significantly $\left(d f=3, x^{2}=11.78, p=0.008, n=7\right)$ across trimesters of pregnancy and pre-conception concentrations (Fig. 1, Table 1). Post-hoc analysis revealed that compared to pre-conception (follicular phase) values, fecal iEA concentrations were significantly higher $\left(\mathrm{T}^{+}=0, \mathrm{p}=0.016, \mathrm{n}=7\right)$ in the $1^{\text {st }}$ trimester (Fig.1, Table 1) whereas no difference was found between pre-conception concentrations and the $2^{\text {nd }}$ and the $3^{\text {rd }}$ trimester, respectively (follicular phase vs. $2^{\text {rd }}$ trimester: $T^{+}=16, p=0.812, n=7$; follicular phase vs. $3^{\text {rd }}$ trimester: $T^{+}=16, p=0.812, n=7$; Fig. 1 , Table 1).

Table 1: Study females, parity, fetal sex and fecal iEA concentrations during the luteal and the follicular phase and trimesters of pregnancy (values are given in mean \pm SEM $\mu \mathrm{g} / \mathrm{g}$ dry weight).

\begin{tabular}{lccccccc} 
& & & Luteal & Follicular & \multicolumn{2}{c}{ Trimester of pregnancy } \\
Female ID & Parity & Fetal sex & phase $^{\text {b }}$ & phase & first & second & third \\
\hline fs10 & $\mathrm{mp}$ & $\mathrm{m}$ & $1.3 \pm 0.3$ & $1.0 \pm 0.2$ & $2.4 \pm 0.4$ & $1.2 \pm 0.2$ & $1.7 \pm 0.4$ \\
$\mathrm{fs} 06$ & $\mathrm{mp}$ & $\mathrm{m}$ & - & $3.1 \pm 0.3$ & $3.3 \pm 0.4$ & $2.9 \pm 0.5$ & $1.7 \pm 0.2$ \\
$\mathrm{fs} 07$ & $\mathrm{mp}$ & $\mathrm{m}$ & $1.0 \pm 0.3$ & $1.0 \pm 0.2$ & $3.5 \pm 0.6$ & $1.7 \pm 0.1$ & $1.8 \pm 0.1$ \\
$\mathrm{fs} 05$ & $\mathrm{pp}$ & $\mathrm{f}$ & $0.8 \pm 0.2$ & $1.0 \pm 0.1$ & $2.7 \pm 0.7$ & $0.7 \pm 0.1$ & $1.3 \pm 0.2$ \\
$\mathrm{fs} 11$ & $\mathrm{mp}$ & $\mathrm{f}$ & - & $1.2 \pm 0.1$ & $2.8 \pm 0.9$ & $2.8 \pm 0.2$ & $1.4 \pm 0.4$ \\
$\mathrm{fs} 08$ & $\mathrm{mp}$ & $\mathrm{f}$ & - & $1.4 \pm 0.2$ & $2.2 \pm 0.2$ & $0.9 \pm 0.1$ & $0.8 \pm 0.1$ \\
$\mathrm{fs} 03^{\mathrm{a}}$ & $\mathrm{mp}$ & - & - & $4.1 \pm 1.6$ & $5.1 \pm 1.2$ & $2.0 \pm 0.3$ & $2.0 \pm 0.3$ \\
\hline
\end{tabular}

${ }^{a}$ died around the estimated timing of parturition

${ }^{\mathrm{b}} 4$ females conceived during their first cycle 


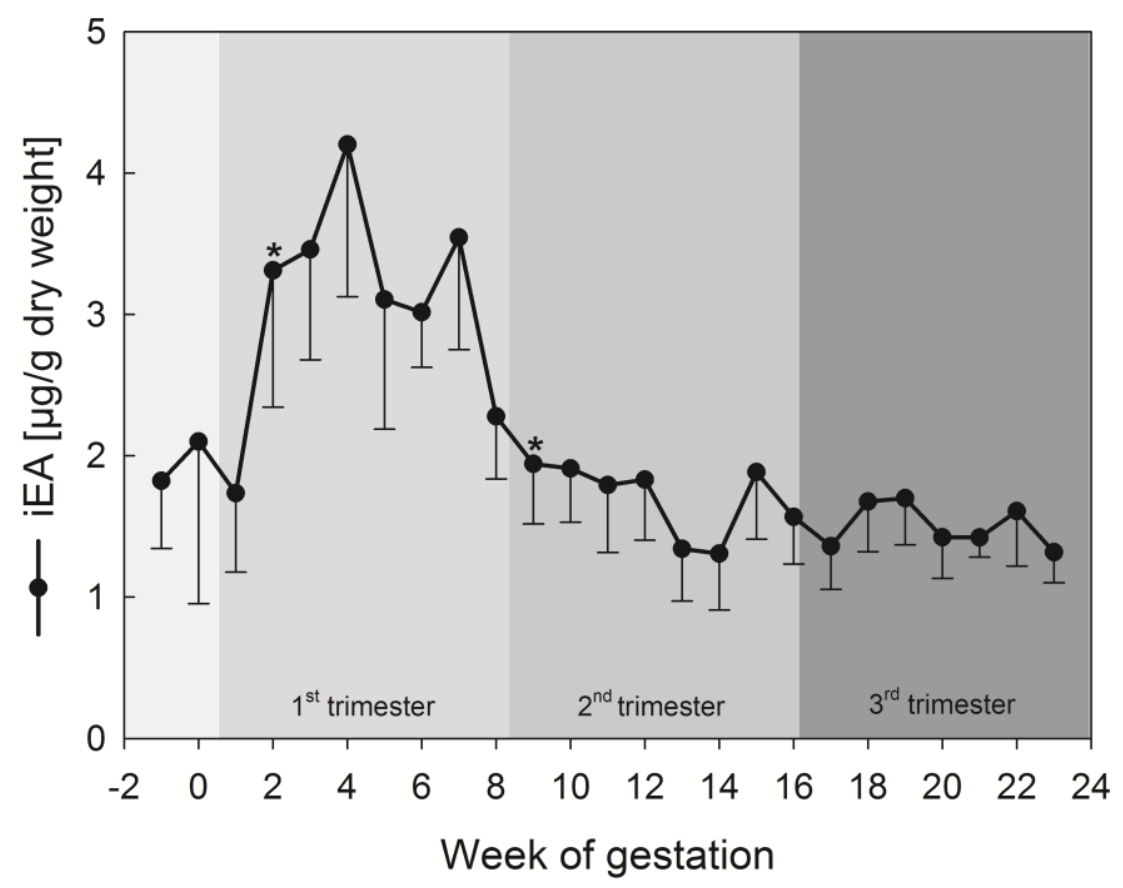

Figure 1: Patterns of fecal androgen (iEA) excretion during pregnancy in wild female Assamese macaques. Data were derived from seven females and are presented as weekly mean+SEM. Asterisks indicate (1) the significant $(p=0.031)$ increase in iEA concentrations between weeks $1-2$, and (2) the significant $(p=0.031)$ decrease in iEA concentrations between weeks 7-9.

\section{Fetal sex effects on maternal androgen concentrations}

Three of the seven pregnancies resulted in the birth of male offspring, and three resulted in the birth of female offspring (Table 1). Whereas androgen concentrations did not significantly differ ( $1^{\text {st }}$ trimester: $\mathrm{t}=1.30, \mathrm{df}=3.10, \mathrm{p}=0.1 ; 2^{\text {nd }}$ trimester: $\mathrm{t}=0.56, \mathrm{df}=3.72, \mathrm{p}=0.3$ ) according to the sex of the fetus in the $1^{\text {st }}$ and $2^{\text {nd }}$ trimester of gestation, fetal sex had a significant effect $(\mathrm{t}=3.01, \mathrm{df}=2.13, \mathrm{p}=0.044, \mathrm{n}=6$; Fig. 2; Table 1) on maternal androgen production during the $3^{\text {rd }}$ trimester of pregnancy in that females carrying male fetuses (mean $\pm S E M: 1.7 \pm 0.03 \mu \mathrm{g} / \mathrm{g}$ ) exhibiting significantly higher fecal iEA concentrations compared to those carrying female fetuses (mean \pm SEM: $1.2 \pm 0.2 \mu \mathrm{g} / \mathrm{g}$ ).

One study female (fs03) died a few days before the estimated time of parturition, and thus the sex of the infant remained unknown; however based on the mother's mean androgen level in the $3^{\text {rd }}$ trimester (mean \pm SEM: $2.0 \pm 0.3 \mu \mathrm{g} / \mathrm{g}$ ), the fetus was presumably a male (Table 1). 


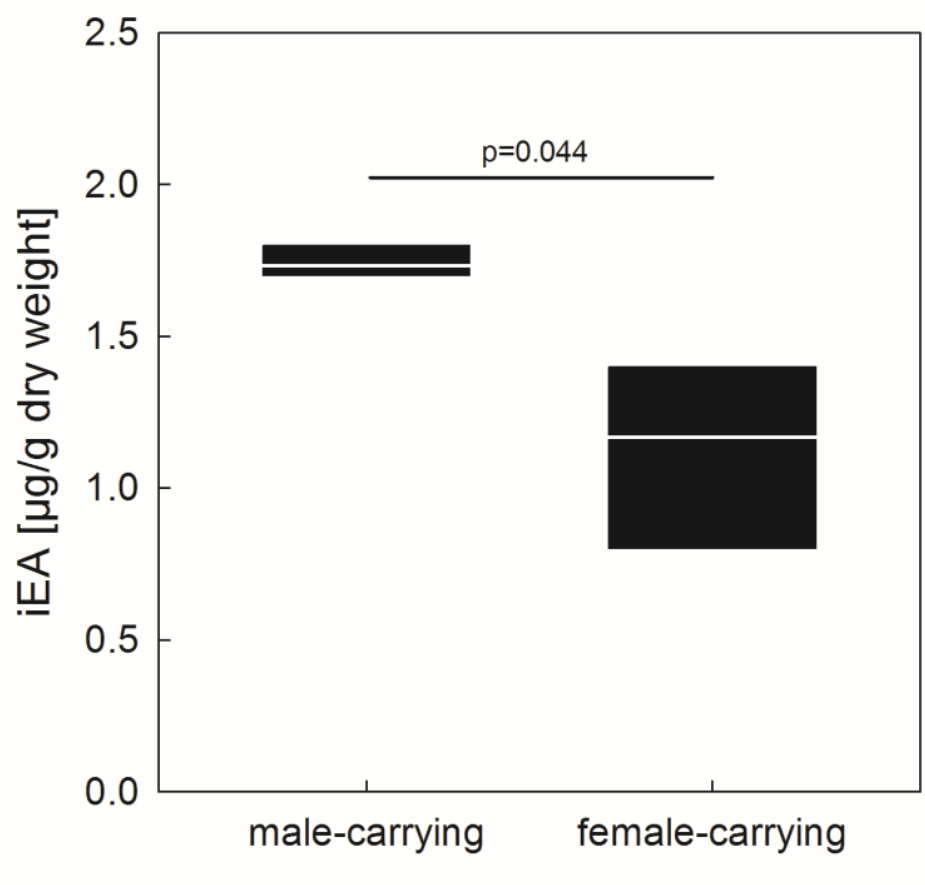

Females

Figure 2: Box-plot depicting range and mean (white line) maternal fecal androgen (iEA) excretion in the third trimester of pregnancy according to fetal sex $\left(n_{1}=3, n_{2}=3, p=0.044\right)$.

\section{DISCUSSION}

This first study on excreted androgens in wild Assamese macaque females revealed clear differences in fecal androgen concentrations throughout gestation, with fecal iEA concentrations being significantly elevated during early pregnancy (first trimester) compared to the pre-conception period and later stages of pregnancy. Furthermore, we determined that there was a significant effect of fetal sex on maternal androgen excretion during the third trimester of pregnancy with mothers carrying a male fetus exhibiting nearly $50 \%$ higher concentrations than mothers carrying a female fetus. Although part of our data is limited by a small sample size, the data as a whole extend and confirm previous findings on a few other cercopithecine primate species and strongly suggest that androgen production during Assamese macaque pregnancy is not solely derived from maternal sources, but also influenced by androgens of fetal origin.

The significant rise in androgen production occurred as early as the second week of gestation, followed by a gradual decline towards pre-pregnancy concentrations around the transition from the first to the second trimester, i.e. between weeks 7 to 9 of gestation. It is unlikely that the observed changes in fecal androgen concentrations were owing to seasonal cues (e.g. change in day length) because in all females androgen output increased in the first 
trimester irrespective of whether individual females conceived early (October) or late (January) in the mating season. Moreover, in the two females that conceived earliest and latest, peak androgen levels were recorded in almost the same week relative to conception (weeks 4 and 5), confirming that stage of gestation rather than seasonal variables were responsible for the pattern of androgen output seen.

Our results reflect the predicted pattern based on our knowledge derived from the few other cercopithecine monkeys (with comparable gestation lengths, see (Kappeler \& Pereira, 2003) studied in this respect. In bonnet macaques (Macaca radiata), for example, serum testosterone concentrations increase during early pregnancy, peak at day 33 , and decline to baseline concentrations at day 50 (Rao \& Kotagi, 1983). Similarly, in yellow baboons (Papio cynocephalus) blood concentrations of testosterone and androstenedione increase significantly between days 4 and 20 , continue to rise to day 33 , and then decline to luteal phase concentrations by day 50 (Castracane \& Goldzieher, 1983). In olive baboons (Papio anubis), peripheral concentrations of androstenedione and testosterone were significantly elevated during days 10-19 and 34-40, and declined to baseline concentrations by day 60 (Hodges et al., 1984). Thus, in terms of the temporal dynamics in early gestational androgen production, Assamese macaques appear to resemble other cercopithecine primate species; however, a finer grained analysis involving more frequent sampling would be needed to further investigate this.

The mechanisms underlying androgen production during gestation in Assamese macaques are not known, however, elevated androgens during pregnancy in primates are likely to be under regulation of chorionic gonadotropin (CG), as in several species increasing androgen concentrations coincide with increasing CG concentrations (e.g. Castracane \& Goldzieher, 1983; French et al., 2010; Rao \& Kotagi, 1983). This link has also been confirmed experimentally in baboons (Papio cynocephalus) in which CG administration during the non-pregnant late luteal phase resulted in elevated testosterone and androstenedione concentrations (Castracane \& Goldzieher, 1983). A regulation of maternal androgen production by CG would be in line with our finding that androgen concentrations decline and remain low after the first third of gestation when increased CG production in macaques and other cercopithecines usually cease. Although previous studies did not provide data on maternal androgen production throughout the entire gestation as reported here, we would predict that this pattern of low androgens after day 50-60 of gestation is typical for macaques and other cercopithecines. Although the sources of origin of the androgens excreted by our pregnant study females remain to be established, it is likely that they are derived from multiple sources, including the ovary, corpus luteum, placenta, and presumably adrenal (although probably a minor contributor) (humans: Castracane et al., 1998). Given that in cercopithecine monkeys the luteal-placental shift (i.e. the time when the 
ovary and corpus luteum are not required to maintain pregnancy) is not established before the end of the third week of gestation (Castracane \& Goldzieher, 1986; Hodgen \& Itskovitz, 1988), it is likely that the elevated concentrations of early gestational androgens are solely of ovarian origin (humans: Castracane et al., 1998). However, androgens are elevated throughout the first 2 months of pregnancy, suggesting a placental origin of androgens during later stages of the first trimester (baboons: Hodges et al., 1984). Whether the decline in maternal androgen concentrations to pre-conception concentrations from week 7-9 onwards reflects a decreased placental activity in androgen production during the second and third trimester or is linked to an increase in aromatization of androgens to estrogens before excretion and/or a feedback action between androgens and estrogens (see Hodges et al., 1984) remains unclear. However, maternal fecal estrogen concentrations in pregnant Assamese macaques do not rise during the time when androgens decline (Fürtbauer et al., 2010), suggesting that a decrease in placental androgenesis may be more likely.

From a more practical point of view, the rise in fecal androgens shortly after conception potentially allows to non-invasively detect early pregnancy in this species, given that androgen concentrations are substantially (2-4 fold, see Table 1 ) lower in the luteal phase of a non-conception cycle. From our data it can be inferred that pregnancy determination based on a change in androgen concentrations may in fact be made within 2 weeks of conception and thus earlier than one based on progestogens (and estrogens) which cannot be done before 3-4 weeks after fertilization. Serial sampling around the peri-ovulatory period into early gestation is presumably necessary to achieve this, however, because a simple cut-off threshold to distinguish non-pregnant from pregnant values is probably difficult to define in view of the inter-individual variability observed in fecal androgen concentrations across females (see Table 1).

Because fetal testes produce and secrete androgens, which, in turn, may affect maternal androgen concentrations (e.g. Altmann et al., 2004; Drea, 2011; Duer et al., 2002; Meulenberg \& Hofman, 1991), we investigated a potential effect of fetal sex on maternal iEA concentrations. Indeed we found a significant effect of fetal sex on maternal androgen concentrations in our pregnant Assamese macaque females in the direction predicted, i.e. mothers carrying male fetuses exhibited significantly elevated fecal androgen concentrations during late pregnancy. Given that our sample size for this comparison was small and therefore the possibility that the fetal sex related differences found may reflect normal variability in androgen levels cannot be ruled out completely, this result should be considered preliminary. However, the fact that the difference in maternal androgen levels with respect to fetal sex was statistically significant even with this small sample size together with similar findings in other species (see below) suggests that there is presumably a true effect. A fecal androgen measure may thus potentially allow determining infant sex prenatally, although the 
overlap between late gestation androgen levels of male- and female carrying mothers may make determination of a cut-off value in this respect difficult. Additional analyses of more pregnancies are certainly required to investigate this potential diagnostic value further.

Concerning timing, the fetal sex effect on maternal iEA concentrations was not observed before the onset of the third trimester of pregnancy and thus about 2.5 months later compared to when the fetal testes in macaques starts to produce androgens (day 35-50; Resko \& Ellinwood, 1981). A study on fecal androgens in pregnant baboons (Papio cynocephalus) revealed a comparable effect (with a similar small sample size) of fetal sex on maternal androgen production during the third trimester, but, unfortunately, the study did not investigate androgen production during earlier stages of gestation (Altmann et al., 2004). Recently, Drea (2011) reported male fetal androgen effects on maternal androgen concentrations also especially in the third trimester of pregnancy in ring-tailed lemurs (Lemur catta). In contrast, in humans, maternal testosterone concentrations in women carrying male fetuses gradually increase during pregnancy and are significantly higher compared to women carrying female fetuses during the second half of gestation (Meulenberg \& Hofman, 1991). Thus, it seems that a fetal sex effect on androgen production in the mother is less pronounced during early stages of pregnancy in primates more generally. This may not be surprising given the high amount of placental androgens produced during this period (see Fig. 1) compared to the presumably low amount of fetal androgens secreted at this time. Alternatively, but not mutually exclusive, the lower resolution of any fecal hormone measure compared to the measurement of hormones in blood may have prevented us and others from detecting fetal sex effects during earlier stages of gestation.

\section{ACKNOWLEDGMENTS}

We thank the National Research Council of Thailand (NRCT) and the Department of National Parks, Wildlife and Plant Conservation (DNP) for permission (No. 0004.3/3618) to conduct this study. We are grateful to J. Prabnasuk, K. Nitaya, M. Kumsuk and K. Kreetiyutanont (Phu Khieo Wildlife Sanctuary) for their cooperation and permission to carry out this research. We thank A. Koenig and C. Borries (Stony Brook University), who developed the field site at Huai Mai Sot Yai, for their support. We are thankful to A. Heistermann, M. Swagemakers, D. Bootros, T. Kilawit, A. Chunchaen and P. Saisawatdikul, S. Jomhlotwong, S. Rogahn and W. Nuagchiyos. We also thank N. Bhumpakphan, W. Eiadthong (Kasetsart University) and W. Brockelman (Mahidol University). 



\section{CHAPTER 3}

\section{Concealed fertility and extended female sexuality in a non-human primate (Macaca assamensis)}

Fürtbauer, I., Heistermann, M., Schülke, O., \& Ostner, J. (submitted) Concealed fertility and extended female sexuality in a non-human primate (Macaca assamensis). PLoS ONE.

\section{ABSTRACT}

In numerous primates living in mixed-sex groups, females display probabilistic cues of fertility to simultaneously concentrate paternity to dominant males while diluting it amongst others as a means to reduce the risk of infanticide and to increase male care for offspring. A few species, however, lack these cues and potentially conceal fertility from males; yet, to date, little is known about mating patterns and their underlying proximate mechanisms in such species. Here, we investigated mating activity and sexual consortships relative to female reproductive state in wild Assamese macaques (Macaca assamensis), a species where females lack prominent anogenital swellings and copulation calls. During two mating seasons (2837 contact hours) we recorded sexual and social behaviors, sexual consortships, and collected 1178 fecal samples ( $n=15$ females) which were analyzed for progestogen concentrations to assess female reproductive state and to determine the timing of ovulation and conception. Although mostly conceiving in their first ovarian cycle, females were sexually receptive throughout the entire 4-month mating season, and within-cycle mating frequencies were not increased during fertile phases. Dominant males did not monopolize fertile matings, and consortships by high-ranking males lasted for long periods, which were not exclusively linked to female fertile phases. Furthermore, females copulated promiscuously but not randomly, i.e. for almost every female, matings were concentrated to a certain male, irrespective of male rank. Collectively, we demonstrate that fertility is undisclosed to males. The extreme extended female sexuality facilitated by concealed fertility may allow females to create differentiated mating relationships within a promiscuous mating system. Our study provides important new insight into the plasticity of female sexuality in non-human primates. 


\section{INTRODUCTION}

Sexual selection theory states that due to the asymmetry in parental investment, males should increase their number of offspring by mating with many females whereas females should mate more selectively in order to increase their reproductive success (Bateman, 1948; Trivers, 1972). Contrary to these predictions, however, female promiscuity is widespread among vertebrates and invertebrates (e.g. Arnqvist \& Nilsson, 2000; Panova et al., 2010; Uller \& Ollson, 2008; van Noordwijk \& van Schaik, 2000; Wolff \& Macdonald, 2004). Given the potential costs of this behavior in terms of time, energy, disease and parasite transmission, predation and/or injury inflicted by males (e.g. Arnqvist \& Nilsson, 2000; Jennions \& Petrie, 2000; Lockhart et al, 1996; Matsubara \& Sprague, 2004; Nunn et al., 2000; Reynolds \& Gross, 1990; Smuts \& Smuts, 1993), females are expected to gain compensatory benefits from mating with many males. Polyandrous mating has therefore commonly been associated with procreative benefits as it may ensure insemination, promote sperm competition, increase the genetic variability of offspring, or avoid inbreeding (Cornell \& Tregenza, 2007; Eberhard, 1996; Jennions \& Petrie, 2000; van Noordwijk \& van Schaik, 2000; Small, 1988; Yasui, 1997).

Polyandrous mating during non-fertile stages, however, must serve functions other than reproduction. The most convincing non-procreative explanation (among several hypotheses; see Wolff \& Macdonald, 2004) is that female polyandrous mating is a female counterstrategy to infanticide risks posed by males (Hrdy, 1979; van Noordwijk \& van Schaik, 2000; van Schaik, 2000b; van Schaik \& Janson, 2000; Wolff \& Macdonald, 2004). Primates, in particular, face a high risk of infanticide owing to their slow life histories (van Noordwijk \& van Schaik, 2000). It has been proposed that by mating with many males females may manipulate male paternity assessment as a means to reduce infanticide risks (Borries et al., 1999; Heistermann et al., 2001a; Hrdy, 1979; Manson, 1992; Nunn, 1999a; van Schaik et al., 2000; Small, 1990; Soltis et al., 1999). Male primates usually base their decisions on whether or not to attack an infant on paternity estimates, and thus should be less likely to attack infants of females they have previously mated with (van Schaik et al., 2004; Soltis et al., 2000). Additionally, if paternity estimates are high for certain males, polyandrous mating may secure or increase male care from these males for future infants including protection from infanticide (van Schaik et al., 2000, 2004; Soltis, 2002; Stacey, 1982; Taub, 1980).

At a proximate level, polyandrous mating requires that females cannot be monopolized by a single (dominant) male. Female primates exhibit a number of physiological, morphological and behavioral alterations in order to break the dominant male's monopoly, i.e. to decrease male mating skew (reviewed in Clarke et al., 2009). Old World primates, in particular, exhibit prolonged mating periods within the ovarian cycle, which has been 
attributed to their long follicular phases (e.g. van Schaik et al., 2000). The timing of ovulation varies within these periods, and thus, is unpredictable for males, hence enabling females to mate with multiple partners (e.g. van Schaik et al., 2000). At the same, time females of numerous Old World primates exhibit anogenital (sexual) swellings and/or copulation calls, i.e. cues that enhance female attractivity around the probable timing of ovulation, and thus, in some way, advertise rather than obscure it (Dixson, 1998; Nunn, 1999b).

Numerous studies focusing on these traits have shown that they function as "graded signals" of ovulation probability, enabling females to overcome the "female dilemma" (van Schaik et al., 1999), i.e. on the one hand, to concentrate paternity in dominant males, while on the other hand, confusing it among many males (e.g. Brauch et al., 2007, 2008; Nunn, 1999b; Semple \& McComb, 2000; Semple et al., 2002; van Schaik et al., 1999; Zinner \& Deschner, 2000). In some species, however, females lack these cues (e.g. Dixson, 1998), raising the question whether these females, nevertheless, concentrate paternity in certain males. Unfortunately, to date, for many of these species little is known about female sexuality relative to reproductive state and the underlying proximate mechanisms involved. This study aims to address this important gap in our general understanding of mating systems and reproductive strategies in primates.

Assuming that paternity manipulation may be reached on different levels, the following two scenarios are possible in the absence of overt cues to fertility: (1) Females may exhibit olfactory (e.g. Cerda-Molina et al., 2006) or behavioral indicators of reproductive state (i.e. "behavioral estrus"; Heape, 1900; Dixson, 1998) leading to a similar outcome, i.e. concentration (dominant males) when fertilization is most, and dilution (subordinate males) when it is less likely, respectively (see above). (2) Fecundity may be undisclosed to males, hence preventing monopolization by dominant individuals during fertile periods. Assuming that males, in the latter scenario, are unable to assess when ovulation is likely to be imminent, and hence, must rely on mating history as their only estimate for paternity probability (instead of relating it to female attractivity and ovulation probability), females may be freer but could still show (non-dominant based) preferences for certain males by simply manipulating "perceived" paternities, i.e. distributing their matings non-randomly across males (see van Schaik et al., 2004).

Assamese macaques (Macaca assamensis) provide an excellent model to investigate the proximate mechanisms underlying female reproductive strategies in the context described above. This species belongs to the minority of macaque species (see Maestripieri \& Roney, 2005) where females lack pronounced sexual swellings (Hrdy and Whitten 1987; Fürtbauer et al., 2010) and copulation calls (Fürtbauer, pers. obs.). Assamese macaque females mostly conceive during their first ovarian cycle within the 4-month mating season (Fürtbauer et al., 2010). Despite a clear male dominance hierarchy, male reproductive skew 
is low (Ostner et al., 2008b, 2011; Schülke et al., 2010) indicating that males are unable to monopolize fertile females. Males in this species, however, frequently interact with infants (Ostner \& Schülke, in prep.; pers. obs.) suggesting, according to recent modeling, high paternity estimates (e.g. van Schaik et al., 2004). Yet, there is no information on the characteristics and patterns of female sexual activity in this species, which would provide new insight into female sexuality in primates in general.

Here, we investigate female mating activity and male consortships (as a marker of the males' ability to monopolize fertile females) in a wild group of Assamese macaques and relate it to female reproductive state, as determined from non-invasive fecal hormone analysis, to address (i) how female receptivity is distributed relative to reproductive state, (ii) whether females exhibit behavioral estrus (indicated by increased mating frequencies and male monopolization around ovulation), or whether fertility is undisclosed to males, and (iii) whether female matings are non-randomly distributed across males.

\section{METHODS}

\section{Study site and animals}

The study was carried out at the Phu Khieo Wildlife Sanctuary $\left(157,300\right.$ ha, $16^{\circ} 5^{\prime}-35^{\prime} \mathrm{N}$, $101^{\circ} 20^{\prime}-55^{\prime} E, 300-1,300 \mathrm{~m}$ a.s.I.), north-eastern Thailand, which consists of dry evergreen forest with patches of dry dipterocarp forest and bamboo stands (Borries et al., 2002). During two consecutive mating seasons ( $M S=$ months during which conceptions may occur) data were collected on a fully habituated group (AS) of Assamese macaques. During the MS 2007/2008 (Oct $1^{\text {st }}$ - Jan $31^{\text {st }}$ ) the study group comprised 53 individuals including 12 adult females and 13 males ( 8 adult and 5 large subadult males; for age classes see Ostner et al., 2008b). During the MS 2008/2009 (Oct $1^{\text {st }}-$ Feb $13^{\text {th }}$ ) the group consisted of 55 individuals including 14 adult females and 15 males (10 adult and 5 large subadult males). Data are presented for 15 females which conceived during the respective mating season (11 multiparous and 4 primiparous females; Table I). We selected those females which, based on their reproductive history, were expected to conceive in the respective mating season. In the MS 2007/2008 none of the non-focal females conceived whereas in the MS 2008/2009 two females (fs08 and fs 11) conceived unexpectedly and thus, had not been sampled. 
Table 1: Study females, dominance rank, parity, and number of fecal samples for hormone analysis.

\begin{tabular}{|c|c|c|c|c|}
\hline Mating season & Female & Dominance Rank $^{a}$ & Parity & Number of samples \\
\hline \multirow[t]{7}{*}{ 2007/2008 } & fs03 & 3 & $\mathrm{mp}$ & 72 \\
\hline & fs 05 & 2 & $\mathrm{pp}$ & 73 \\
\hline & fs06 & 4 & $\mathrm{mp}$ & 66 \\
\hline & fs 07 & 10 & $\mathrm{mp}$ & 70 \\
\hline & fs08 & 1 & $\mathrm{mp}$ & 76 \\
\hline & fs 10 & 6 & $\mathrm{mp}$ & 75 \\
\hline & fs 11 & 9 & $\mathrm{mp}$ & 79 \\
\hline \multirow[t]{8}{*}{$2008 / 2009$} & $\mathrm{fs} 02$ & 13 & $\mathrm{mp}$ & 76 \\
\hline & fs04 & 14 & $\mathrm{mp}$ & 72 \\
\hline & fs09 & 9 & $\mathrm{mp}$ & 79 \\
\hline & fs 13 & 6 & $\mathrm{mp}$ & 85 \\
\hline & fs 14 & 4 & $\mathrm{pp}$ & 88 \\
\hline & fs 15 & 10 & $\mathrm{pp}$ & 80 \\
\hline & fs 18 & 2 & $\mathrm{pp}$ & 93 \\
\hline & fs 19 & 8 & $\mathrm{mp}$ & 94 \\
\hline Total & & & & 1178 \\
\hline
\end{tabular}

\section{Behavioral observations}

The study group was followed daily from dawn until dusk during the two mating seasons resulting in 2,837 contact hours with the animals (11.1 \pm 0.7 contact hours per day). Various researchers and assistants (2.5 \pm 0.7 observers per hour; all trained by I.F.) collected data on social and sexual interactions using ad libitum and focal animal sampling (20-30 min; evenly distributed throughout the day and across females), which were pooled for further analyses and to determine for each female whether she was sexually receptive (i.e. mating regardless of fertility) on a given day. For all observed copulations $(n=1280)$ we noted the date, the time, the identities of the individuals, and whether the male ejaculated or not. Ejaculatory copulations could easily be distinguished from non-ejaculatory copulations due to a typical and pronounced pre-ejaculatory pause in male pelvic thrusts. In addition, if observed, we noted whether copulations were male- or female-initiated using approach behavior $(<1 \mathrm{~m})$ immediately prior to the mating as criterion. In order to control for potential rank effects on mating behavior (e.g. Dixson, 1998), female dominance rank was established based on the exchange of clear submissive signals, i.e. "silent bared teeth" (van Hooff, 1967) and "make room" (Ostner et al., 2008b). 
To indirectly assess the males' ability to assess female reproductive state, and hence to monopolize fertile females, we collected data on sexual consortships, i.e. spatio-temporal associations between a male and a female. Consorts were characterized by the pair staying in close proximity $(<10 \mathrm{~m})$, coordinating their movements, grooming, and mating. Consorts lasted from 1 to 49 days.

\section{Hormone analysis}

Fecal hormone analysis to assess female reproductive state and timing of ovulation and conception has been described in detail by Fürtbauer et al. (2010). In brief, we collected on average $4.6 \pm 0.5$ samples per week directly after defecation from each selected female (1178 samples in total, Table I). Samples were stored at $-20^{\circ} \mathrm{C}$ until hormone analysis. Following hormone extraction from freeze-dried and pulverized samples (Fürtbauer et al., 2010), fecal extracts were measured for concentrations of progestogens (20a-dihydroprogesterone; 20aOHP) using a validated microtiterplate enzyme immunoassay (EIA; Fürtbauer et al., 2010). Sensitivity of the assay at $90 \%$ binding was $1.5 \mathrm{pg}$. Intra- and interassay coefficients of variation, calculated from replicate determinations of high- and low-value quality controls were $7.5 \%$ and $13.1 \%$ (high) and $9.2 \%$ and $16.7 \%$ (low).

\section{Assessment of fertile phases and conceptions}

As described in Fürtbauer et al. (2010), we determined the timing of ovulation/conception in our study females by using the defined post-ovulatory rise in fecal progestogen levels, taking into account the fecal excretion lag time. This approach has been successfully applied for monitoring female reproductive status, including timing of ovulation/conception and the female fertile phase, in a variety of primate species in a wide range of taxa (e.g. capuchin monkeys: Carosi et al., 1999; langurs: Ziegler et al., 2000; chimpanzees: Emery \& Whitten, 2003; macaques: O'Neill et al., 2004; Engelhardt et al., 2004; Heistermann et al., 2008; baboons: Higham et al., 2008; callitrichids: Heistermann et al., 1993). We considered day -3 relative to the day of the defined progestogen increase (day 0 ) as the most likely day of ovulation/conception. Because of the possible variability in the temporal relationship between ovulation and the post-ovulatory fecal progestogen rise (Shideler et al., 1993) and since daily samples during the peri-ovulatory period were not always available, data on the timing of ovulation/conception can be expected to include an error of 1-2 days. We defined the fertile phase as a 5-day period including days -2 and -3 (relative to day 0 ) plus the three preceding days to account for sperm longevity in the female reproductive tract (Behboodi et al., 1991). 


\section{Data analyses}

Non-parametric tests were applied using SPSS 17.0 (SPSS Inc.), with the level of significance set at alpha $=0.05$. All statistical tests were two-tailed. Female dominance hierarchy was assessed using the I\&SI method as implemented in MATMAN ${ }^{\mathrm{TM}}$ 1.1.4 (Noldus 2003) based on 375 agonistic interactions. Due to the death of one female (fs03) in June 2008 , and the addition of three primiparous females to the data set in 2008/2009 (fs14, fs 15 , and fs18), both mating seasons were treated separately (Table I).

To determine differences in daily copulation frequencies relative to reproductive state we performed two Generalized Linear Mixed Model (GLMM) analyses with Poisson-distributed error variances in $\mathrm{R}$ ( $\mathrm{R}$ Development Core Team 2008; package Ime4). As assumed by the models, overdispersion was not significant. We used reproductive state and female dominance rank as predictor variables. In the first model, encompassing the entire mating season, reproductive states were defined as follows: "n" (non-fertile= acyclic and cyclic stages prior to conception; i.e. the period between the first observed copulation and the day before the onset of the fertile phase leading to conception), "fert" (fertile= the 5-day fertile phase), and "p" (pregnant= the period between the first day after the fertile phase of the conception cycle and the last observed copulation). In order to investigate within-cycle differences in daily copulation frequencies we performed a second model for which reproductive states were defined as follows: "pre" (prefertile= the five days preceding the fertile phase), "fert" (fertile= the 5-day fertile phase), and "post" (postfertile= the five days following the fertile phase). In both models, we included "female ID" and "mating season", i.e. either $2007 / 2008$ or $2008 / 2009$ as random factors, and applied dummy-coding using "fert" as reference category.

To investigate the distribution of matings across males we used Nonacs's skew calculator (2003). We computed B indices and probability levels to test whether observed B values are due to random chance (Nonacs, 2000). B values of 0 indicate randomly distributed mating, negative values suggest mating is more equally shared than random and positive values indicate greater than random skew.

\section{RESULTS}

\section{Female ovarian cycles and conceptions}

Out of 15 study females, twelve conceived during the first and three during their second ovarian cycle (all three in 07/08). For 13 females we could hormonally determine the precise timing of their fertile phases ( $n=16$ fertile phases). Due to infrequent fecal sampling in 
February 2009 (i.e. the end of the mating season), the exact timing of fertile phases could not be determined for the remaining two females (fs13 and fs 14; Table I) which are therefore not included in some further analyses. However, both females conceived in the $2^{\text {nd }}$ week of February 2009, and furthermore, our hormone data (i.e. frequent sampling from October to January) revealed that both females conceived during their first cycle. Conceptions occurred 28 to 98 days after the start of the mating season.

\section{Female receptivity relative to reproductive state}

Although females mostly conceived in their first ovarian cycle, they mated throughout the entire 4-month mating season (Figure 1). On $87 \%$ of days there was more than one female receptive (MS 07/08: 89\%, range of females being receptive on the same days: 0-7; mean \pm sd: $3.0 \pm 1.7$; median: 3 ; 7 females; MS 08/09: 85\%, range: 0-7; mean \pm sd: $3.1 \pm 1.6$; median: 3; 8 females). Mean duration of receptivity (= number of days between the first and the last observed copulation within the mating season) was $115.4 \pm 7.3$ days in $07 / 08(n=7)$ and $120.9 \pm 12.6$ days in 08/09 $(n=8)$, respectively. Accordingly, females not only mated during their ovarian cycle(s) but also during acyclic and pregnant stages. In total $93.7 \%$ of copulations ( $n=1053 ; 13$ females) were recorded during non-fertile stages $(58.3 \%$ before conception, and $35.4 \%$ during pregnancy), the remaining $6.3 \%$ occurred during female fertile phases. In a GLMM we found that female mating activity varied throughout the mating season, with daily copulation frequencies being significantly higher during fertile (fert, $\mathrm{n}=62$ observations; dummy coding reference category) stages than during pregnancy ( $p, \mathrm{n}=682$ observations), but no significant difference was found between non-fertile ( $n, \mathrm{n}=749$ observations) and fertile phases ( $n$ vs. fert: $z=-0.70, p=0.48 ; p$ vs. fert: $z=-3.33, p<0.001 ; 13$ females; Figure 2A). Female dominance rank was found to influence mating activity, with high-ranking females having higher daily copulation frequencies $(z=-3.41, p<0.001)$.

To investigate within-cycle differences in daily copulation frequencies, i.e. between the prefertile (pre, $\mathrm{n}=65$ observations), fertile (fert, $\mathrm{n}=62$ observations; dummy coding reference category), and postfertile (post, $\mathrm{n}=65$ observations) phase of the conception cycle (as a potential indicator of behavioral estrus within cycles) we performed a second GLMM and found no significant differences between any of the three stages (pre vs. fert: $z=-0.53$, $\mathrm{p}=0.60$; fert vs. post: $\mathrm{z}=-0.91, \mathrm{p}=0.36 ; 13$ females; Figure $2 \mathrm{~B}$ ). 


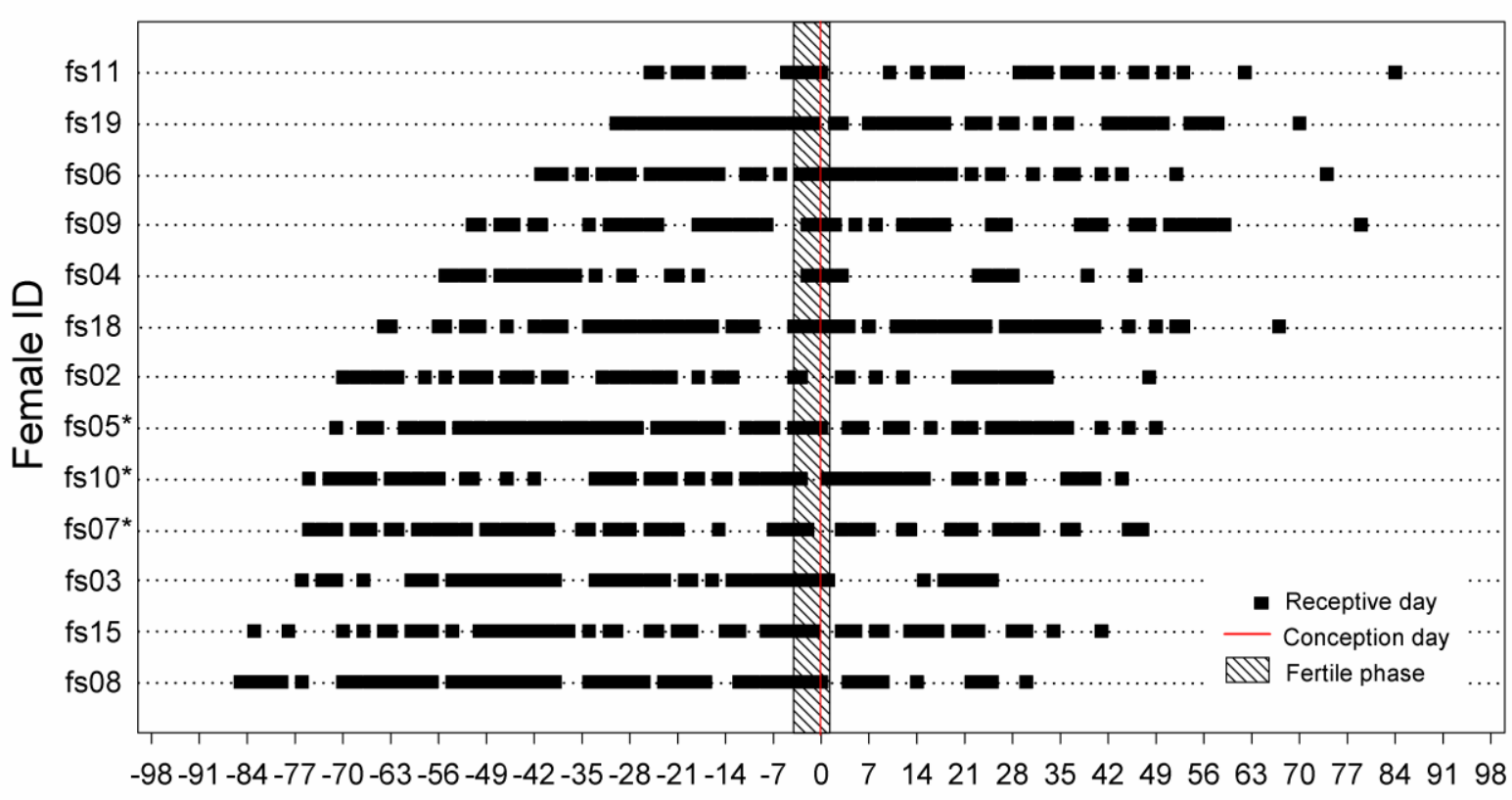

Days of receptivity relative to the day of conception

Figure 1: Female receptivity in relation to the hormonally determined timing of conception. Females marked with an asterisk conceived during the second, others during the first cycle of the mating season. Black squares indicate receptive days. The shaded area denotes the females' fertile phase.

\section{Matings}

Out of 1180 copulations with information on ejaculation $89.4 \%$ were ejaculatory. Of all copulations for which approaches were known, $69.7 \%$ were initiated by the female $(n=278$, median: $73.3 \%$, range across females: 39.3\%-100.0\%; no female rank effect: Spearman's rho $=-0.065, p=0.82, n=15)$. We found no significant differences in female initiation of copulations with regard to male rank (alpha and beta versus lower ranking; Wilcoxon signed ranks test: $Z=-0.369, p=0.71, n=15$ ) and age (adult versus large subadult; Wilcoxon signed ranks test: $Z=-0.682, p=0.50, n=15)$. Mean number of matings per female during the entire mating season was $84.7 \pm 30.9$ in 07/08 (range: $37-127$, median: 73.0 ) and $85.9 \pm 31.8$ in 08/09 (range: 39-144, median: 84.5). Daily copulation frequencies did not differ between multiparous $(n=11)$ and primiparous $(n=4)$ females (Mann-Whitney $U$-test: $U=12, p=0.23$ ). 

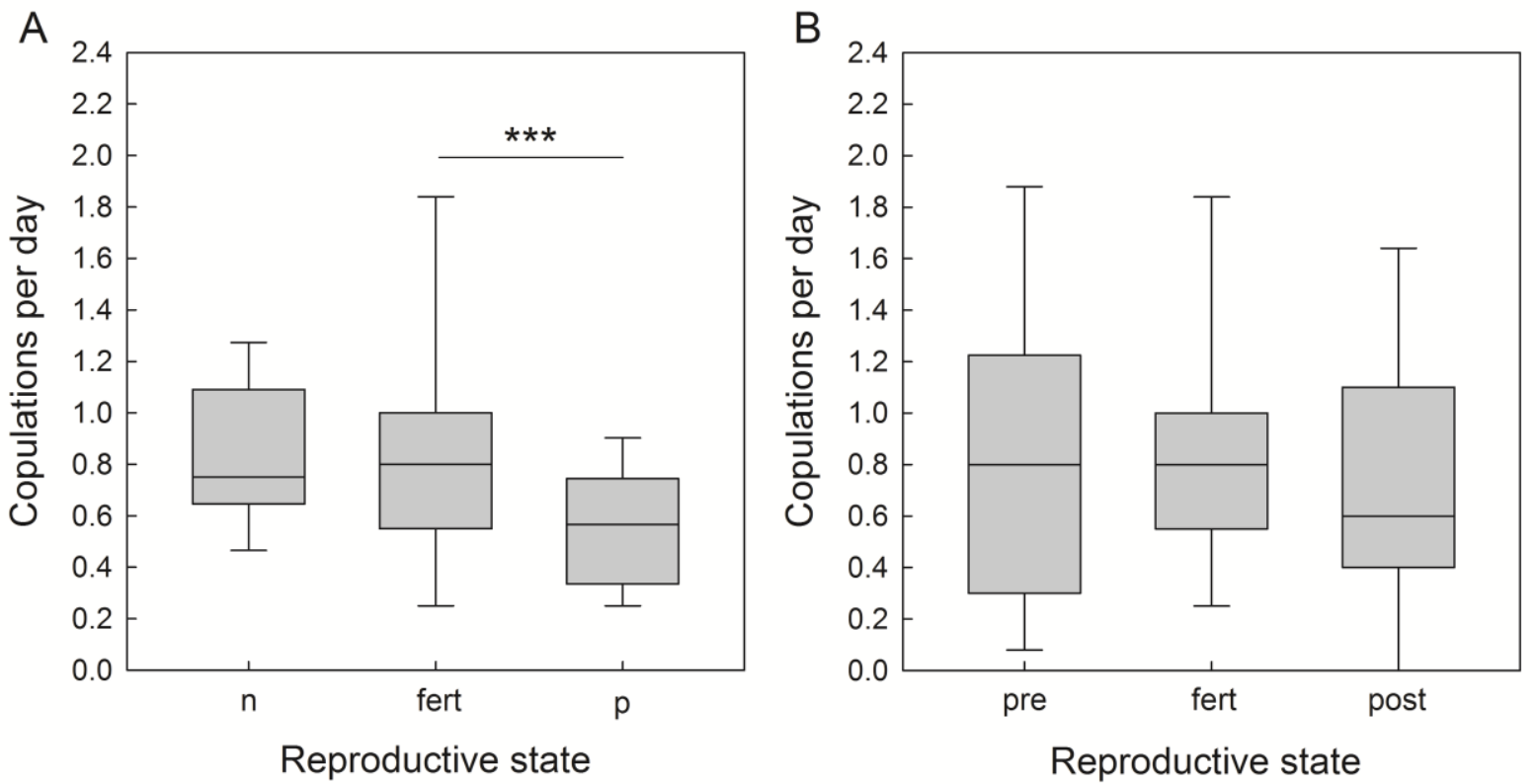

Figure 2: Female daily copulation frequencies in relation to reproductive state during $(A)$ non-fertile $(n)$, fertile (fert), and pregnant $(p)$ stages during the entire mating season $(N=13$ females, two mating seasons) and (B) the prefertile (pre), fertile (fert), and postfertile (post) phase of the conception cycle (i.e. the five days preceding the fertile phase, the five-days fertile phase, and the five days following the fertile phase). For detailed definitions see methods. The boxes indicate medians (line) and upper and lower quartiles. The whiskers indicate the 90th and 10th percentiles. ${ }^{* * \star} p<0.001$

\section{Mating partners}

Out of 211 possible male-female mating dyads 185 have been observed. Thus, on average females mated with $87.5 \pm 10.6 \%$ of all present adult and large subadult males (range: $61.5 \%$ $100 \%$; median: $92.3 ; n=15$; number of mating partners per female: MS 07/08: mean \pm sd:

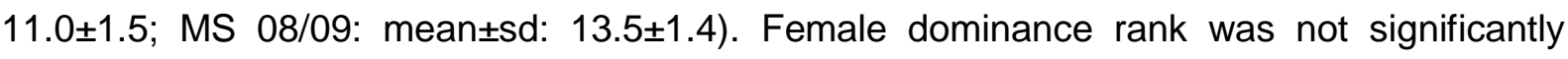
correlated with the number of mating partners (Spearman's rho=-0.115, $p=0.684, n=15$ ). However, female copulations were non-randomly distributed across male mating partners (Figure 3: ranked separately for each female; two mating seasons: MS 07/08: 7 females, 13 males; MS 08/09: 8 females, 15 males). With each female, many males (mean \pm sd: $8.8 \pm 2.4$, range: 4-12, median: 9) obtained low (<10\%) mating proportions, and only a few males (mean \pm sd: $3.4 \pm 1.0$, range: $2-5$, median: 3 ) obtained higher (>10\%) proportions. The category "most mated male", i.e. the male a given female copulated most often with, included 8 different, high- and low-ranking male individuals (MS 07/08: 5 different males, $n=7$ females; MS 08/09: 6 different males, $n=8$ females), indicating no general mating bias towards certain (e.g. top-ranking) males. The most mated male received $24.3 \% \pm 9.8 \%$ of a female's total copulations (range: 12.8\%-44.9\%; median: 20.6\%; 15 females). Skew calculations revealed 
that these inequalities did not occur by random chance. The average B index across all females equaled $0.05 \pm 0.04$ ( $n=15$; range: $0.001-0.139)$ and, overall, was significantly greater than expected by chance $(p<0.001)$. Specifically, significant mating biases were found in 12 females ( 11 females: $p<0.001 ; 1$ female: $p<0.02$ ), two females showed a trend towards a mating bias ( $f s 11: p=0.06$ and $f s 04: p=0.08$, respectively) and for one female matings were distributed randomly across males ( $B$ index not significant; fs15: $p>0.3$ ).

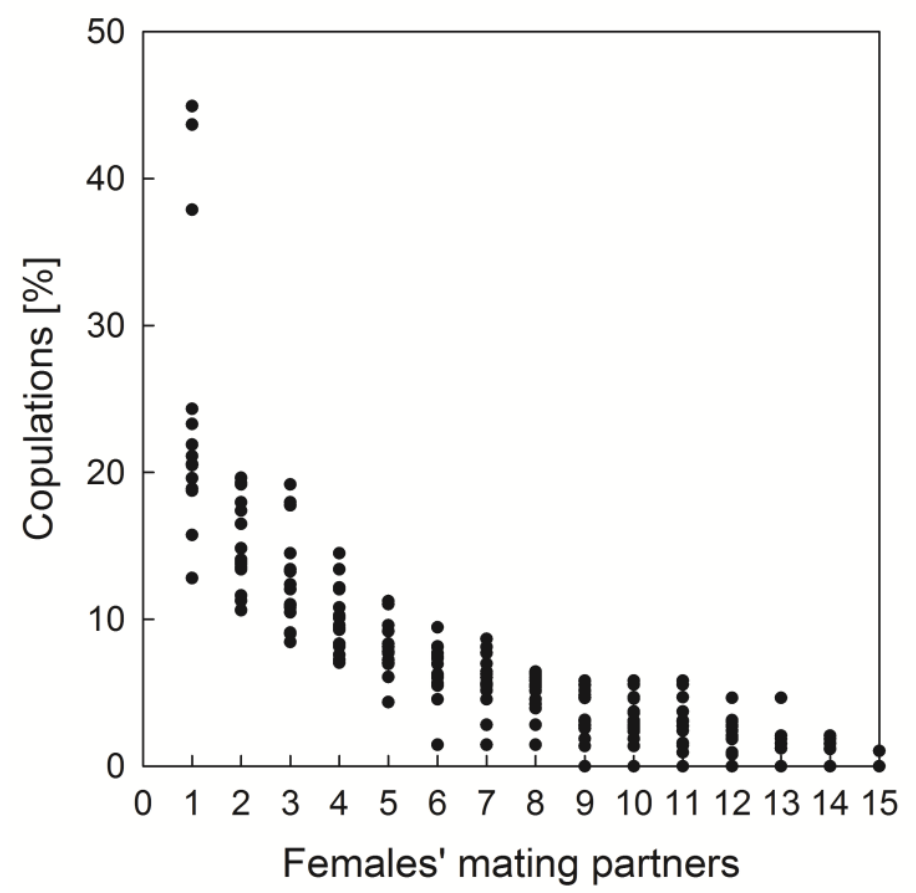

Figure 3: Distribution of copulations across the females' mating partners. Data are combined for two mating seasons (MS 07/08: 7 females, 13 males; MS 08/09: 8 females, 15 males). Note that not all females mated with all males, and that males are ranked separately for each female, i.e. numbers do not denote individuals, e.g. $1=$ the most mated male for each female (including 8 different, high-and low-ranking, adult and large subadult males), $2=$ the second most mated male, etc.

Females mated with, on average, 2.2 males (range: 1-4) during the fertile phase of their conception cycle ( $n=13$ females). Only 3 females mated most frequently with their most mated male during the fertile phase of the conception cycle (all 3 were in consort with the alpha male; $n=11$ females with significant mating skew). An analysis of within-cycle matings for non-consorted (i.e. not largely monopolized) females revealed that females did not mate more often with high-ranking males (i.e. the two top-ranking males) during the fertile phase compared to the pre- and postfertile phase (Friedman test: $d f=2, x^{2}=3.818, p=0.15, n=6$ ). Four of these females never mated with one of the two top-ranking males during their fertile phase. 


\section{Consortships}

Two thirds of the 15 females engaged in long sexual consortships $(n=12)$, ranging from 7 to 49 days, with one of the three top-ranking males (Figure 4). The consorting pair usually stayed within the main group, and some females occasionally sneaked copulations with other males. We found a non-significant trend for consorted females to have less mating partners (median: 1.0) during fertile phases of conception cycles compared to non-consorted females (median: 3.0; Mann-Whitney-U-test: $U=9.5, n_{1}=6, n_{2}=7, p<0.08$ ). Of the 12 observed long consorts, five occurred during non-fertile stages (4 during acyclic stages: fs08, fs 13 , and fs 14 , Figure 4; 1 during pregnancy: fs 18 , Figure 4B). Only 6 consorts covered the female's fertile phase of the conception cycle, and one female was consorted during the fertile phase but did not conceive (Figure 4). Thus, taken together, in 9 cases ( $n=15$ females), females were not consorted by a male around the time of conception.
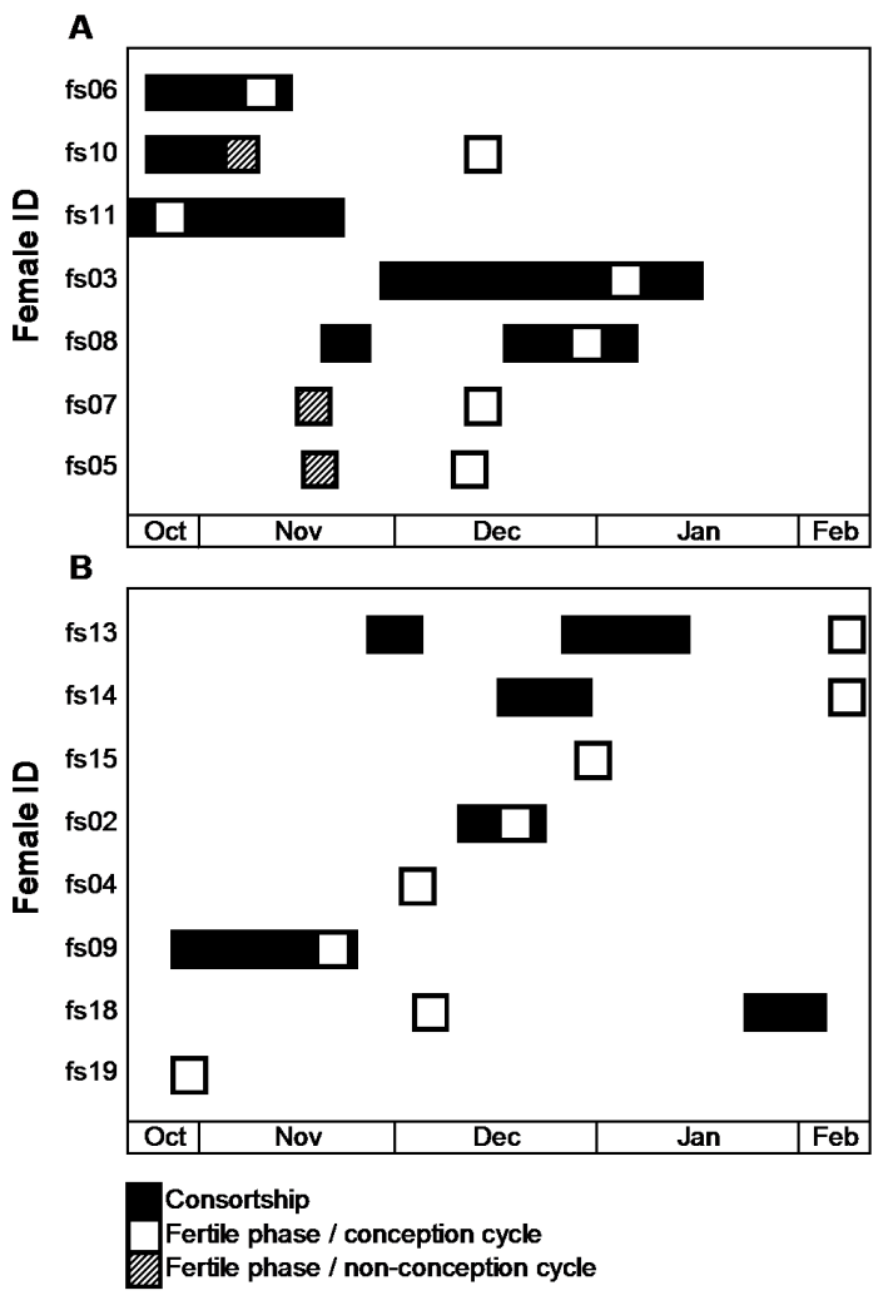

Figure 4: Sexual consortships in relation to the timing of hormonally determined female fertile phases. Data are presented for MS 07/08 (A) and MS 08/09 (B). White boxes indicate conception cycles and shaded boxes non-conception cycles, respectively. 


\section{DISCUSSION}

In this first study on female sexuality in wild Assamese macaques, we show evidence for concealed fertility, extended female sexuality, and female promiscuity. However, copulations were non-randomly distributed across males irrespective of male rank. The potential implications of these rather unusual results shed new light on female reproductive strategies and deserve further discussion.

In contrast to most mammalian species (e.g. McCarthy \& Becker, 2002; Nelson, 2005), mating frequencies did not differ among pre-fertile, fertile and post-fertile phases of the ovarian cycle indicating that in Assamese macaques female reproductive hormones do not play a major role in influencing female attractivity and sexual activity around ovulation. This, together with the lack of apparent visual or auditory manifestations of female fertility, and the fact that the majority of fertile phases was not monopolized by dominant males (see below), suggests that fertility is concealed in this species (without implying an "active" concealment). We cannot completely exclude the possibility, however, that olfactory cues may provide certain information on female reproductive status (e.g. Macaca arctoides; Cerda-Molina et al., 2006), although the mating data suggest that, if such cues do exist, their precision in signaling the female fertile phase is probably poor. Ejaculate production and ejaculatory matings are energetically costly (Dewsbury, 1982; Thomsen et al., 2006), and frequent ejaculations can result in a substantial decrease in sperm number per ejaculate (Preston et al., 2001; Marson et al., 1989) which may compromise fertility (Preston et al., 2001). Thus, if males were able to assess female fertility precisely, one would expect them to focus their reproductive effort to the female fertile phase and dominant males to monopolize fertile matings which, however, was not the case (also reflected in low alpha male paternity outcome; Schülke et al., 2010). Instead, male Assamese macaques invest energy, time and sperm (89\% ejaculatory copulations) in matings with females that cannot conceive, i.e. in females outside their fertile phase ( $94 \%$ non-procreative mating), suggesting that they are unable to discern when a female is fertile.

Usually, in most cercopithecine primates, females display a variety of cues that males may use to assess female ovarian state, with only a few potential exceptions, i.e. species in which ovulation is potentially concealed (e.g. Semnopithecus entellus: Heistermann et al., 2001a; but see Ostner et al., 2006; Cercopithecus aethiops: Andelman, 1987; but see Burt, 1992). Among macaques, Tibetan macaques (Macaca thibetana), the Assamese macaque's closest relatives (Hoelzer \& Melnick, 1996), show very similar mating and consort patterns (see below) but, unfortunately, no accompanying endocrine data are available so that the relationship between these behavioral patterns and the timing of ovulation remains unexplored (Li et al., 2005). 
Strong support that female fertility in Assamese macaques is undisclosed to males comes from the pattern of sexual consortships, i.e. close, spatial associations between a male and a female that usually serve female mate-guarding by males around ovulation as seen in many primate species (Brauch et al., 2008; Engelhardt et al., 2004; Heistermann et al., 2001a, Ostner et al., 2006; for review see Dixson, 1998). Here, we recorded long sexual consortships (ranging from one up to six weeks) between only some of the females and highranking males (Ostner et al., 2011), but in the majority of conception cycles (60\%) females were not consorted at all by a male. Consorts occurred during all reproductive stages (including non-fertile ones), indicating that males probably cannot discriminate between female reproductive states differing in terms of reproductive significance for increasing male reproductive success. If males were able to detect when females are likely to ovulate, we would, contrary to our results, expect consortships to be shorter, and to be linked to female fertile phases, and not to occur during acyclic stages (see Dixson, 1998; Manson, 1997). Further, although some female fertile phases overlap (which may diminish male monopolization potential), we would expect a higher proportion of consorted fertile phases, given their temporal distribution (see Fig. 5). Under such conditions of apparent inability of males to discern female fertility, high ranking males may choose to make the "best of a bad job" by consorting a few females for extended periods to secure at least some paternities (Ostner et al., 2011).

Generally, females could benefit from concealed fertility whenever they receive maledelivered benefits through extended sexuality (Thornhill \& Gangestad, 2008). As previously shown, female Assamese macaques do not exhibit regular ovarian cycles outside the mating season, and mostly conceive during their first cycle (Fürtbauer et al., 2010). Nevertheless, irrespective of the timing of conception, female mating activity was extended over the entire mating season, i.e. all females were receptive long before the onset of cyclic ovarian activity (up to three months before ovulation, see Fig. 1), and continued mating upon conception, i.e. during the first 2-3 months of pregnancy, resulting in a considerable amount of nonprocreative mating (see Soltis, 2002). This is in line with our conclusion that female mating activity in Assamese macaques is not under cyclic hormonal control.

In principle, extended female sexuality serves to obtain benefits from males (either from primary partners within pair-bonds or from multiple males; Arnqvist \& Nilsson, 2000; Hrdy, 1979; Gangestad \& Thornhill, 2008; Rodriguez-Gironés \& Enquist, 2001; Soltis, 2002; Thornhill \& Gangestad, 2008). It has long been argued that in potentially infanticidal species females benefit from mating with multiple males by reducing the chance that males attack their future offspring (Hrdy, 1979; van Schaik \& Janson, 2000; van Noordwijk \& van Schaik, 2000; Wolff \& Macdonald, 2004). Infanticide is extremely costly for female primates, and in many species females exhibit prolonged mating periods (within ovarian cycles) and engage 
in situation-dependent receptivity (e.g. Hrdy, 1979; van Noordwijk \& van Schaik, 2000). Concurrently, in numerous primate species females exhibit cues of ovulation probability to overcome the 'female dilemma', i.e. to bias paternity in the dominant male to protect their future offspring, and simultaneously dilute it among all males to prevent infanticidal attacks (e.g. Nunn, 1999b; van Schaik et al., 1999, 2000, 2004).

Assamese macaque females lack overt cues to fertility, exhibit extended sexuality, and are highly promiscuous. But why not choose the dominant male? Investing heavily in diluting paternity among resident males instead of concentrating it in the dominant male (assuming him to be the best infant protector) may be favorable for females if (1) alpha male tenure is short (resulting in a shorter period to protect the infant), and (2) if the risk of infanticide mainly arises from within the group (by a resident male which rises to alpha position) instead of from an immigrant male taking over the alpha rank (e.g. Clarke et al., 2009; Pradhan \& van Schaik, 2008). Reproductive skew is significantly reduced in insidecompared to outside-takeover species, reflecting female tendencies toward polyandry in inside-takeover species (Clarke et al., 2009). Empirically investigating the effects of takeover modes requires comparative long-term data which are generally not available for many species including Assamese macaques. Given the low mating and paternity skew in our study population (Ostner et al., 2011; Schülke et al., 2010), we may expect a high probability of takeover from inside and consequently also large benefits from paternity dilution in Assamese macaques.

Obviously, prolonged mating periods facilitate promiscuity, the extreme extended female sexuality observed in this study, however, is puzzling and it appears unlikely that females "need" four months to mate with each male in order to achieve maximum paternity dilution, hence suggesting other non-mutually exclusive functions than raising benefits from multiple males. In human females, for example, extended sexuality appears to enhance benefits from primary partners within pair-bonds (Gangestad \& Thornhill, 2008; Thornhill \& Gangestad, 2008). Is it possible that extended female sexuality in Assamese macaques has a similar function? Interestingly, although females copulated with virtually all males, they distributed their matings non-randomly across their partners, i.e. females mated with many males on few occasions, while at the same time frequently copulating with only a few males irrespective of male rank. Almost every female had a different most frequent mating partner including high- and low-ranking, adult and large subadult males who obtained on average a quarter of a female's total copulations. Thus, we envision that extended sexuality in Assamese macaques may also function to obtain more direct benefits from primary partners. In other words, by frequently copulating with a particular male during a prolonged period of time (facilitated by concealed fertility), females and males may create special relationships despite and within the promiscuous mating system. 
Male Assamese macaques appear to have no information on female fertility state (see above), and thus, in order to assess paternity certainty, cannot temporally relate matings to ovulation probability. Accordingly, mating frequencies may be their only estimates, hence function as probabilistic signals of perceived paternity (see Moscovice et al., 2010). Future studies are needed to test whether male-infant interactions (male Assamese macaques frequently interact with, protect and care for infants; Ostner \& Schülke, unpub. data; pers. obs.) and/or male-female interactions outside the mating season reflect these non-dominance based mating biases (e.g. Busse, 1985; Moscovice et al., 2010; Smuts, 1985). Also, it remains to be investigated whether mating biases indicate female preferences or are caused by male coercive behavior (see Muller \& Wrangham, 2009; Muller et al., 2011). Furthermore, genetic analyses are needed to determine whether primary partners are genetic fathers, and whether female mating biases are related to genetic traits (e.g. major histocompatibility complexes; e.g. Schwensow et al., 2008; Setchell et al., 2010b).

\section{ACKNOWLEDGMENTS}

We thank the National Research Council of Thailand (NRCT) and the Department of National Parks, Wildlife and Plant Conservation (DNP) for permission (No. 0004.3/3618) to conduct this study. We are grateful to J. Prabnasuk, K. Nitaya, M. Kumsuk, and K. Kreetiyutanont (Phu Khieo Wildlife Sanctuary) for their cooperation and permission to carry out this study. We thank A. Koenig and C. Borries (Stony Brook University), who developed the field site at Huai Mai Sot Yai, for their support. We are thankful to A. Heistermann and J. Hagedorn for technical assistance in hormone analysis, and to M. Swagemakers, D. Bootros, T. Kilawit, A. Chunchaen and P. Saisawatdikul, S. Jomhlotwong, S. Rogahn and W. Nuagchiyos for their support in the field. We thank M. Franz for his help with the GLMMs. We also thank N. Bhumpakphan, W. Eiadthong (Kasetsart University) and W. Brockelman (Mahidol University) for their support and cooperation. The project was supported with funds from the National Geographic Society, Max-Planck Society and the German Initiative of Excellence to the University of Göttingen. 


\section{CHAPTER 4}

\section{You mate, I mate: Macaque females synchronize sex not cycles}

Fürtbauer, I., Mundry, R., Heistermann, M., Schülke, O., \& Ostner, J. (submitted). You mate, I mate: Macaque females synchronize sex not cycles. PLOS ONE.

\section{ABSTRACT}

Extended female sexuality in species living in multimale-multifemale groups appears to enhance benefits from multiple males. Mating with many males, however, requires a low female monopolizability, which is affected by the spatiotemporal distribution of receptive females. Ovarian cycle synchrony potentially promotes overlapping receptivity if fertile and receptive periods are tightly linked. In primates, however, mating is often decoupled from hormonal control, hence reducing the need for synchronizing ovarian events. Here, we test the alternative hypothesis that females behaviorally coordinate their receptivity while simultaneously investigating ovarian cycle synchrony in wild, seasonal Assamese macaques (Macaca assamensis), a promiscuous species with extremely extended female sexuality. Using fecal hormone analysis to assess ovarian activity we show that fertile phases are randomly distributed, and that dyadic spatial proximity does not affect their distribution. We present evidence for mating synchrony, i.e. the occurrence of the females' receptivity was significantly associated with the proportion of other females mating on a given day. Our results suggest social facilitation of mating synchrony which explains (i) the high number of simultaneously receptive females and (ii) the low male mating skew in this species. Active mating synchronization may serve to enhance the benefits of extended female sexuality, and may proximately explain its patterning and maintenance. 


\section{INTRODUCTION}

Extended female sexuality (i.e. non-conceptive receptivity) has been reported for numerous vertebrate and invertebrate species and is most likely part of a female strategy to obtain male-delivered benefits (e.g. Arnqvist \& Nilsson, 2000; Hrdy, 1979, 2000; Gangestad \& Thornhill, 2008; Rodríguez-Gironés \& Enquist, 2001; Soltis, 2002; Thornhill \& Gangestad, 2008). Many examples come from pair-living species including birds and mammals (e.g. Birkhead \& Møller, 1992; Rodríguez-Gironés \& Enquist, 2001; Thornhill \& Gangestad, 2008). Here, extended sexuality appears to predominantly function to gain material assistance delivered by primary partners (e.g. Gangestad \& Thornhill, 2008, Rodríguez-Gironés \& Enquist, 2001). Conversely, in mammals with multimale-multifemale social organization, such as many non-human primates, extended female sexuality often co-occurs with promiscuity, and thus, has been argued to enhance benefits from multiple males, namely to refrain from infanticide or to provide care for future infants (e.g. Borries et al., 1999; Heistermann et al., 2001a; Hrdy, 1979, 2000; Soltis, 2002; van Schaik et al., 2000, 2004).

Females in multimale-multifemale groups, however, usually face the problem of being monopolized by a single (dominant) male (e.g. van Schaik et al., 1999, 2000, 2004). Thus, for extended sexuality to be effective, i.e. to enhance polyandrous mating, at least some degree of female behavioral freedom is required, which can be achieved by increasing the number of simultaneously sexually active females. Female reproductive synchrony, i.e. the spatiotemporal clustering of receptive females, limits the degree to which females can be monopolized by a single male (e.g. Altmann, 1962; Cowlishaw \& Dunbar, 1991; Emlen \& Oring, 1977; Ims, 1988, 1990; Kutsukake \& Nunn, 2006; Nunn, 1999a; Ostner et al., 2008a; Ridley, 1986), hence enabling them to mate with multiple males and also to exert alternative mate choices (e.g. Eberhard, 1996; Hrdy, 2000; Knowlton, 1979; Soltis, 2002).

The temporal coordination of reproductive activity can be facilitated by seasonal breeding (likely to be regulated through external cues; e.g. Brockman \& van Schaik, 2005; Knobil \& Neill, 2006) because several females may get into breeding condition during a short period of time (e.g. Ridley 1986; Cowlishaw \& Dunbar 1991), or it can be achieved through active synchronization (see below). This may sometimes make it difficult to disentangle the potential ecological and social factors underlying reproductive synchrony, in particular in highly seasonal species (e.g. Ims, 1990; Pereira, 1991).

Studies focusing on mammalian reproductive synchrony are generally based on the assumption that females achieve synchrony by synchronizing (and asynchrony by desynchronizing) their ovarian cycles (e.g. Gattermann et al. 2002; French \& Stribely, 1987; Handelmann et al., 1980; Matsumoto-Oda et al., 2007; McClintock, 1971; McClintock, 1978; Monfort et al., 1996; Pereira, 1991; Setchell et al., 2010a; Tobler et al., 2010; Wallis, 1985). 
Studies on rodents revealed mixed evidence for ovarian cycle synchrony (e.g. Rattus norvegicus: McClintock, 1978; Schank, 2001a; Mesocricetus auratus: Handelmann et al., 1980; Gattermann et al., 2002). Similarly, in primates, the results remain controversial and inconclusive. Apart from a few studies (Homo sapiens: McClintock, 1971; Pan troglodytes: Wallis, 1985; Leonthopithecus rosalia: French \& Stribley, 1987; but see Schank, 2001b,c) most investigations found no evidence for ovarian cycle synchrony (e.g. Papio hamadryas: Tobler et al., 2010; Homo sapiens: Strassman, 1997; Mandrillus sphinx: Setchell et al., 2010a; Leontopithecus rosalia: Monfort et al., 1996) or report asynchrony (e.g. Papio hamadryas: Zinner et al., 1994; Pan troglodytes: Matsumoto-Oda et al., 2007; Lemur catta: Pereira, 1991).

Several primate-typical reproductive features may potentially explain the lack of ovarian cycle synchrony observed in most species: First, and in contrast to most mammals (e.g. Knobil \& Neill, 2006), female receptivity in primates is usually decoupled from hormonal control (e.g. Hrdy, 1979; Dixson, 1998; Wallen, 1990). Second, most primates exhibit extended mating periods within ovarian cycles (some Old World monkeys mate throughout their complete cycle), a phenomenon that has been attributed to prolonged follicular phases (e.g. van Noordwijk \& van Schaik, 2000; van Schaik et al., 2000). Third, primate females often show an increased number of cycles to conception, extending their total mating period (e.g. Clarke et al., 2009), hence increasing the chance to overlap in mating activity with other females. Fourth, male knowledge about female fertility status usually is imperfect (e.g. Heistermann et al., 2001a; Deschner et al., 2004). In general, the "need" for ovarian cycle synchrony in order to reduce male monopolization potential should decrease with (i) the degree of emancipation of sexual behavior from hormonal control, (ii) increasing length of the mating period, and (iii) increasing unpredictability of fertility (note that these factors are not independent). To illustrate an extreme: in species where fertility is undisclosed to males, and females potentially mate throughout and beyond their cycles, synchronizing ovarian events becomes unnecessary to achieve overlapping receptive periods. Furthermore, ovarian cycle synchrony cannot explain synchronous receptivity during acyclic stages (e.g. pregnancy).

Here, we focus on an alternative, non-mutually exclusive, hypothesis that females synchronize their mating activity. Assuming that male monopolization potential is affected by the spatiotemporal distribution of receptive females (e.g. Altmann, 1962; Cowlishaw \& Dunbar, 1991; Emlen \& Oring, 1977; Ridley, 1986), and that mating is decoupled from hormonal control, females may mate non-randomly with respect to time, in order to avoid male monopolization. On a proximate level, and in contrast to ovarian cycle synchrony which is assumed to be regulated by pheromonal cues (e.g. rats: McClintock, 1984; humans: Stern \& McClintock, 1998; Russel et al., 1980), mating synchrony may be achieved through behavioral coordination (Coussi-Korbel \& Fragaszy, 1995), i.e. females may respond to the 
mating activity of other females in the group. In other words, the probability of a given female to copulate may be affected by the number of other females mating.

To our knowledge, no study has yet attempted to investigate whether females synchronize their mating activity independent of ovarian cycle synchrony. Here, we use hormone and mating data obtained from wild female Assamese macaques (Macaca assamensis) to test this hypothesis. Using permutation procedures and Linear Mixed Effects Models we investigate whether females synchronize their mating activity. We simultaneously examine whether female fertile phases are significantly more synchronous or asynchronous than expected from a random distribution using permutation procedures. Also, we examine whether dyadic spatial proximity affects the temporal distribution of fertile phases, to test the hypothesis that those females who spend more time in close proximity cycle more closely together (e.g. see McClintock, 1984; Stern \& McClintock, 1998).

Assamese macaques breed seasonally, and females mostly conceive in their first ovarian cycle (Fürtbauer et al., 2010) with fertile phases overlapping to some extent (Fürtbauer et al., submitted). Females exhibit no apparent coordination between receptive and fertile periods as they become sexually receptive (i.e. sexually active) in unison (up to 3 months before the onset of cyclic ovarian activity) and mate, at low frequencies, throughout the 4-month mating season and even into pregnancy (Fürtbauer et al., submitted). As a consequence, on nearly $90 \%$ of mating season days there is more than one female receptive. The extreme extended female sexuality together with concealed fertility (Fürtbauer et al., submitted) diminishes male monopolization potential which is reflected in an unusually low alpha male mating skew (17.5\%; Ostner et al., 2011). Despite a high degree of promiscuity (i.e. females mate with virtually all males) females exhibit non-dominance based mating biases towards different (high- and low-ranking) male individuals (Fürtbauer et al., submitted). Only top ranking males engage in long sexual consortships, not linked to female fertile phases (Fürtbauer et al., submitted; Ostner et al., 2011). The strong seasonality of the species may account for some overlap in both fertility and receptivity. The extreme nonconceptive mating activity beyond ovarian cyclicity, however, is puzzling and making the setting ideal to test whether females behaviorally coordinate their mating activity.

\section{METHODS}

\section{Subjects}

During two consecutive mating seasons (MS) behavioral and hormone data were obtained from a wild group of Assamese macaques at the Phu Khieo Wildlife Sanctuary (157,300 ha, $16^{\circ} 5^{\prime}-35^{\prime} \mathrm{N}, 101^{\circ} 20^{\prime}-55^{\prime} \mathrm{E}, 300-1,300 \mathrm{~m}$ a.s.I.), north-eastern Thailand. During the MS 07/08 
(Oct $1^{\text {st }}$ - Jan $31^{\text {st }}$ ) the study group comprised 53 individuals including 13 males and 12 adult females, seven of which conceived and data are presented for. During the MS 08/09 (Oct $1^{\text {st }}$ - Feb $13^{\text {th }}$ ) the group consisted of 55 individuals including 15 males and 14 adult females, ten of which conceived and eight of which data are presented for (two females conceived unexpectedly, hence had not been sampled). No subject was included twice in the study (i.e. $\mathrm{n}=15$ females).

\section{Behavioral data}

The study group was followed from dawn to dusk (2,837 contact hrs; $11.1 \pm 0.7$ contact $\mathrm{hrs} /$ day) throughout the two mating seasons combining ad libitum and focal observations. We recorded social and sexual behaviors (for details on female sexuality see Fürtbauer et al., submitted), and conducted proximity scans $(n=5627)$ every ten minutes during focal observations by noting all females within $5 \mathrm{~m}$ of the focal female. Female dominance rank was established based on the exchange of clear submissive signals, i.e. "silent bared teeth" (van Hooff, 1967) and "make room" (Ostner et al., 2008b). Both mating seasons were treated separately because one female died in June 2008, and three primiparous females were added to the data set in the MS 08/09. In order to control for rank effects, female dominance hierarchy was assessed using the I\&SI method as implemented in MATMAN ${ }^{\mathrm{TM}}$ 1.1.4 (Noldus 2003). We then standardized ranks to a range from 0 (lowest ranking) to 1 (highest ranking), and with the females considered in the study evenly spaced between these two values.

\section{Assessment of fertile phases}

Fecal hormone analysis to assess ovulation and fertile periods has been described in detail by Fürtbauer et al. (2010, submitted). In brief, we collected on average ( $\pm s d) 4.6 \pm 0.5$ samples per week from each study female. Following hormone extraction from freeze-dried samples, extracts were measured for concentrations of progestogens (20a-dihydroprogesterone; 20aOHP) using a validated microtiterplate enzyme immunoassay (EIA; Fürtbauer et al., 2010). Sensitivity of the assay at $90 \%$ binding was $1.5 \mathrm{pg}$. Intra- and interassay coefficients of variation, calculated from replicate determinations of high- and low-value quality controls were $7.5 \%$ and $13.1 \%$ (high) and $9.2 \%$ and $16.7 \%$ (low).

As described in Fürtbauer et al. (2010), the timing of ovulation was determined by using the defined post-ovulatory rise in fecal progestogen levels, taking into account the fecal excretion time lag. Day -3 relative to the day of the defined progestogen increase (day 0 ) was considered as the most likely day of ovulation. The fertile phase was defined as a 5-day period including days -2 and -3 (relative to day 0 ) plus the three preceding days (c.f. 
Fürtbauer et al., submitted). For two females the exact timing of the fertile phase could not be determined but could be narrowed down to the first half of February 2009.

\section{Ovarian cycle synchrony}

To test for synchrony of fertile periods we used the approach of Matsumoto-Oda et al., (2007). We measured the Synchrony-Index (SI) as described therein and tested its significance based on 1,000 permutations in which we included the original data as one permutation. Units of permutation were the fertile periods and the intervals between them (permuted separately). Other than in Matsumoto-Oda et al. (2007) we imposed a restriction on the permutation algorithm which ensured that the total duration of the period lasting from the beginning of the first to the end of the last fertile period was kept constant. In case a female went through two fertile periods ( $n=3$ females, MS 07/08; see Fürtbauer et al., 2010) we kept the time interval between them constant and just permuted the two fertile periods. Since synchrony could in principle be smaller as well as larger (i.e. asynchrony versus synchrony) we determined two one-tailed p-values. In the MS 08/09 the precise timing of the fertile periods for 2 females was not known (see above). Hence, we constructed data sets representing all possible combinations of timings of the fertile periods of these two females and tested each of them using the approach described above. For these we report the average $p$-value. Permutation tests were calculated using a program written by R.M. (we did not use a linear model for this analysis because fertile periods last 5 continuous days, hence making the occurrence of fertile and non-fertile days within periods clearly non-independent).

To test whether close spatial proximity between females affected the timing of their fertile phases (c.f. McClintock, 1984; Stern \& McClintock, 1998), we first determined for each dyad the proportion of scans at which the two females were no more than five meters apart. Furthermore, we determined for each dyad the absolute number of days elapsed between the onsets of their fertile phases. We then correlated the two matrices using a Mantel test (Sokal \& Rohlf, 1995). This test was exact (i.e. enumerating all possible permutations of the data) and based on Spearman's rho as the test statistic. For the MS 08/09 where the exact onset of fertile phases could not be determined for two females, we used the approach described above (averaging the result for all possible combinations of onset days). Mantel tests were calculated using a program written by R.M.

\section{Mating synchrony}

To test whether females synchronized days on which they copulated we used the following approach: First, we calculated for each female and day the proportion of the other females 
which copulated that day. We then used a Generalized Linear Mixed Model (GLMM; Baayen, 2008) to test whether the probability of a given female to copulate (response variable: yes/no) on a given day was influenced by the proportion of other females copulating that day. Besides the proportion of females copulating we also included the conception status (preand post-conception), its interaction with the former variable, and the females' rank as fixed effects. In addition, we included female ID and MS (07/08 and 08/09) as random effects.

The response variable in the model was likely to show temporal autocorrelation unexplained by the fixed effects included. Thus, the assumption of independent residuals was likely to be violated (i.e. neighboring residuals being more similar than more distant ones) devaluating the reliability of the model. Hence, we decided to explicitly incorporate temporal autocorrelation into the model using the following approach: We first ran the model with all fixed and random effects included and derived the residuals from it. Then, for each data point we calculated an 'autocorrelation term' as the average of the residuals of all other data points of the same female with the contribution of the residuals being weighted by their time lag to the particular data point. The weight followed a normal distribution, with its standard deviation determined by minimizing the AIC (Burnham \& Anderson, 2002) of the GLMM including the autocorrelation term as an additional fixed effect.

Regarding significance testing we first determined the significance of the full model (including all fixed effects, the interaction, the autocorrelation term and random effects) as compared to the corresponding null model (including only the autocorrelation term and the random effects) using a likelihood ratio test (Dobson, 2002). Only if this revealed significance we considered the significance of the individual predictors. P-values of main effects we considered only if they were not included in a significant interaction.

We calculated the GLMM in R (version 2.11.1, R Development Core Team, 2010) using the function Imer of the R package Ime4 (Bates \& Maechler, 2010). GLMM's were fitted with binomial error structure and logit link function and likelihood ratio tests were calculated using the $\mathrm{R}$ function anova. To enhance the reliability of this likelihood ratio test we used maximum likelihood estimation in the mixed model (argument REML of the function Imer set to FALSE). Significance of the individual fixed effects was determined based on the $z-$ and $p$ values provided by Imer. The autocorrelation term was calculated using a function written by R.M. and the minimization of the AIC to find the best fitting standard deviation of the weight function for the autocorrelation term was done using the $R$ function optimize.

Additionally, we used a permutation procedure similar to that described for ovarian cycle synchrony (see above). We did this because the assessment of P-values for fixed effects can be unreliable in Mixed Models (Bolker et al., 2008). We first measured the variance in mating synchrony as described in Matsumoto-Oda et al. (2007) and then used the following approach: we first identified, separately for each female, sections of consecutive 
days all of which comprising at least one copulation, and sections of consecutive days none of which comprising at least one copulation. We then permuted these sections, separately for sections with and without copulations. In addition, we permuted data only for days between the first and the last day with copulation. Since there were two days without observations in the MS 07/08 and one day without observations in the MS 08/09, we subdivided the study period into three phases in the MS 07/08 and two phases in the MS 08/09, respectively, and permuted data only within these phases. We determined the P-value as the proportion of permutations revealing variance in synchrony being at least as large as that of the original data. We used 1,000 permutations and the original data were included as one permutation.

\section{RESULTS}

\section{Ovarian cycle synchrony}

We investigated patterns of ovarian cycle synchrony during two mating seasons and found no evidence of synchrony or asynchrony of female conceptive fertile phases (5 days; hormonally determined; MS 07/08; $\mathrm{SI}=0.187, \mathrm{p}_{\text {(syn) }}=0.102, \mathrm{p}_{\text {(asyn) }}=0.939 ; \mathrm{MS}$ 08/09: $\mathrm{SI}=0.118$, $\mathrm{p}_{\text {(syn) }}=0.455, \mathrm{p}_{\text {(asyn) }}=0.640$; no error level correction applied). Because in the MS 07/08 three females conceived during their second cycle (all other females in the same year and in the MS 08/09 conceived in their first cycle), we ran the permutation procedure with including the three non-conception cycles, and achieved similar non-significant results $(S I=0.162$, $\left.\mathrm{p}_{(\text {syn) }}=0.2, \mathrm{p}_{(\text {asyn) }}=0.834\right)$. Dyadic spatial proximity of females was not correlated with the interval between the onsets of fertile periods (Mantel test, MS 07/08: $r_{S}=-0.04, n=7$ females, $p=0.84$; MS 08/09, average of 48 tests: $r_{S}=0.22, n=8$ females; $p=0.24$ ).

\section{Mating synchrony}

A GLMM revealed evidence that females synchronized their mating activity. We found an overall significant effect of the entire set of predictor variables included in the model (likelihood-ratio test comparing the fit of the full with that of the null model comprising only the random effects: $x^{2}=22.30, d f=4, N=1927$ days and 15 females, $p<0.001$ ). The probability of a given female to copulate (response variable: yes/no) on a given day clearly increased with the proportion of other females copulating that same day (Table 1). In addition, higher ranking females were more likely to copulate. Female conception status (i.e. pre- or postconception) had no significant effect on the probability to copulate.

Because assessing the significance of fixed effects in the framework of Mixed Models is associated with some uncertainty (Bolker et al., 2008), we backed up our conclusions with 
a permutation test as applied for the synchrony of the fertile phases (see above). We obtained matching results in that sections of consecutive mating days (see methods for details) were more synchronous than expected by chance ( $\mathrm{SI}=0.175, \mathrm{p}=0.002$ ).

Table 1: Factors influencing the probability of a given female to copulate on a given day (binary variable). Female ID $(n=15)$ and season $(n=2)$ were included as random factors. The interaction between the number of other females copulating and conception status was not significant (estimate $\pm S E=-0.05 \pm 0.51, z=-0.10, P=0.92$ ). The numbers presented in the table are from a model not comprising this interaction.

\begin{tabular}{lccc} 
Predictor variable & Estimate \pm SE & z value & $\mathbf{p}$ \\
\hline Intercept & $-1.14 \pm 0.16$ & -7.18 & $<0.001$ \\
Other females copulating & $0.64 \pm 0.24$ & 2.71 & 0.007 \\
Conception status & $0.17 \pm 0.12$ & 1.38 & 0.17 \\
Dominance rank & $0.87 \pm 0.20$ & 4.47 & $<0.001$ \\
Autocorrelation term & $2.00 \pm 0.17$ & 11.45 & $<0.001$ \\
\hline
\end{tabular}

Predictor variables: Proportion of other females copulating, conception status (pre-and postconception, female dominance rank (standardized across the two mating seasons), and autocorrelation term

\section{DISCUSSION}

Our data show that although in Assamese macaques female fertile phases partly overlap (Fürtbauer et al., submitted), those females who spent more time in close proximity did not cycle more closely together (pheromonal hypothesis; e.g. McClintock, 1984; Stern \& McClintock, 1998; but see, e.g. mandrills: Setchell et al., 2010a, chimps: Matsumoto-Oda \& Kasuya, 2005; mouse lemurs: Radespiel \& Zimmermann, 2001), and that the observed ovarian cycle overlap did not deviate from random expectations. In contrast, we provide evidence for significant synchrony of mating activity.

The result that females did not synchronize their cycles is not surprising because specific reproductive and life-history traits may "prevent" females from synchronizing their ovarian cycles. First, Assamese macaque females are anovulatory during the non-mating season and mostly conceive during their first ovulatory cycle within the mating season (Fürtbauer et al., 2010). 'Active' ovarian cycle synchronization, however, is likely to require several cycles (e.g. McClintock, 1971). A recent study on mandrills (Mandrillus sphinx), for example, found evidence for significant female cycle synchrony in only one out of ten years, the year with most cycles (overall and per female) recorded, suggesting a link between ovarian cycle synchrony and the number of cycles to conception (Setchell et al., 2010a). 
Second, as shown recently for Assamese macaques, early conception within the mating season may allow for a 1-year interbirth interval (IBI) whereas females conceiving late in the season are more likely to conceive their next infant after two years (Fürtbauer et al., 2010). Any shifting of ovarian cycles towards the end of the mating season, for the benefit of ovarian cycle synchrony, may have to be paid with a 2-year instead of a 1-year IBI, and thus considerably affects female lifetime reproductive success, and should not be selected for. In line with this, the period between parturition and consecutive conception in Assamese macaque females with 1-year IBIs is relatively constant (265 to 290 days; Fürtbauer et al., 2010), indicating that females are impregnated as soon as they resume cycling (female primates need to attain a critical body weight to resume cycling; e.g. Bercovitch, 1987; Maninger et al., 2000). Third, the main hypothesis to explain ovarian cycle synchrony is to increase the number of simultaneously receptive females in order to decrease male monopolization potential and to mate with multiple males (e.g. Emlen \& Oring, 1977; Kutsukake \& Nunn, 2006; Ridley, 1986). In Assamese macaques, however, fertility is undisclosed to males, and females mate rather continuously throughout the 4-month mating season, i.e. during acyclic, cyclic, and pregnant stages (Fürtbauer et al., submitted). So, females do not need to synchronize their fertile periods because males appear to be unable to recognize them, and because mating is largely decoupled from hormonal control.

The alternative hypothesis tested here is that females actively synchronize their mating not their ovarian activity. We found that the occurrence of a female's receptivity on a given day was significantly associated with the proportion of other females mating that day. Female Assamese macaques start being sexually active in unison with some females mating up to three months before they experience their first ovulatory cycle (Fürtbauer et al., submitted). Our results not only explain the high number of simultaneously receptive females not due to ovarian cycle synchrony and environmental seasonality, but also offer a possible proximate explanation for the patterning and maintenance of extended sexuality as described in the following scenario: In Assamese macaques, conceptions are spread over the mating season, with few conceptions occurring in October, i.e. at the beginning of the mating season (Fürtbauer et al., 2010). The onset of the mating season is likely to be triggered by external cues (e.g. photoperiod; Brockman \& van Schaik, 2005; Knobil \& Neill, 2006) which induce the onset of ovulatory and sexual activity in a few females, namely those who conceived their last infant two years ago (see above). Other females who resume cycling later in the season start being receptive in response to the mating of these females. Thus, receptivity is socially (behaviorally and not via pheromones) mediated and induced before the onset of ovulatory activity and, furthermore, maintained after conception, i.e. during pregnancy. In Assamese macaques $70 \%$ of copulations are female-initiated (Fürtbauer et al. submitted) (and females 
very rarely refuse copulations; IF, pers. obs.), which supports the active nature of the observed female mating synchrony.

Social facilitation of sexual behavior has already been proposed for patas monkeys (Erythrocebus patas; Rowell \& Hartwell, 1978). A study on rhesus macaques (Macaca mulatta) has shown that not only male sexual behavior increased due to the presence of females which were experimentally brought into sexual receptivity during the non-mating season but also untreated females exhibited copulatory behavior (Vandenbergh \& Drickamer, 1974). In blue monkeys (Cercopithecus mitis stuhlmanni), the simultaneous onset of receptivity may also be socially mediated; however, no endocrine data are available in order to rule out ovarian cycle synchrony (Cords et al., 1986). The matting patterns of vervet monkeys (Cercopithecus aethiops; see Andelman, 1985, 1987) and Tibetan macaques (Macaca thibetana; see Li et al., 2005) both closely resembling that of Assamese macaques, suggest a behaviorally coordinated mating activity. It would be rewarding to test whether female receptivity is more synchronous than random in the species mentioned above in order to evaluate the general applicability of the mating-synchrony-hypothesis. Also, the discrepancy between observed and expected female mating overlap in some primate species (see Nunn et al., 2001; Kutsukake \& Nunn, 2006), may, given our results, be explained by active mating and not necessarily ovarian cycle synchrony (which anyway is disputed in primates; e.g. Schank, 2001b). Theoretical support for socially induced mating behavior comes from a recent model on the evolution of increased female sexuality which indicates that sexual behavior outside fertile periods can occur as soon as some fertile females appear in the population (Rodríguez-Gironés \& Enquist, 2001).

On an ultimate level, although not tested here, behavioral coordination (Coussi-Korbel \& Fragaszy, 1995) of sexual activity may have different functions. The two main potential explanations, depending, at least partly, on the social system of the species, relate to (1) female-female reproductive competition and (2) intersexual conflict over paternity concentration. In uni-male groups of western lowland gorillas (Gorilla gorilla), for example, females synchronize post-conception copulations to occur when other females mate, which has been linked to female mating competition (Doran-Sheehy et al., 2009; Stoinski et al., 2009). In contrast, in species with multimale-multifemale social organization, we propose that active mating synchrony may be an effective female strategy whenever paternity concentration in the dominant male is less important than paternity dilution among many males (e.g. inside take-over species; see Pradhan \& van Schaik, 2008), or when females adopt an alternative mate choice strategy not based on male dominance rank (e.g. MHCassociated mating; Schwensow et al., 2008; Setchell et al., 2010b). In Assamese macaques, females are highly promiscuous (i.e. they mate with virtually all males) while at the same time expressing non-dominance based mating biases (towards different males), and mating 
repeatedly with their 'primary partner' (for details see Fürtbauer et al., submitted). Furthermore, in this species, females rarely interfere in copulations of other females (IF, pers. obs.), indicative of female-female competition being an unlikely explanation for the observed mating synchrony. Active mating synchrony, i.e. great flexibility in sexual behavior, throughout the mating season (i.e. pre- and post-conception) appears to diminish largely male monopolization potential and reproductive skew in this species $(17.5 \%$ alpha male mating share; Ostner et al., 2011), which enables females to exert their reproductive strategy, i.e. creating differentiated mating relationships within a promiscuous mating system (see Fürtbauer et al., submitted).

Finally, we call for the use of consistent terminology. Irrespective of conflicting evidence for synchrony in the context of animal reproduction, numerous terms (ovarian-, menstrual-, estrous-, cycle-, female-, receptive-, reproductive-, breeding-, and mating synchrony) have been inconsistently used between and within studies in order to describe any overlap in female reproductive events. Hence, we explicitly stress the differentiation of physiological and behavioral reproductive synchrony given our finding that mating synchrony can occur in the absence of ovarian cycle synchrony.

\section{ACKNOWLEDGMENTS}

We thank the National Research Council of Thailand (NRCT) and the Department of National Parks, Wildlife and Plant Conservation (DNP) for permission (No. 0004.3/3618) to conduct this study. We are grateful to J. Prabnasuk, K. Nitaya, M. Kumsuk, and K. Kreetiyutanont for their cooperation and permission to carry out this study. We thank A. Koenig and $C$. Borries (Stony Brook University), who developed the field site at Huai Mai Sot Yai, for their support. We are thankful to A. Heistermann, J. Hagedorn, M. Swagemakers, D. Bootros, T. Kilawit, A. Chunchaen and P. Saisawatdikul, S. Jomhlotwong, S. Rogahn and W. Nuagchiyos. We also thank N. Bhumpakphan, W. Eiadthong (Kasetsart University) and W. Brockelman (Mahidol University). The project was supported with funds from the National Geographic Society, Max-Planck Society and the German Initiative of Excellence to the University of Göttingen. 


\section{GENERAL DISCUSSION}

Sexual conflict is a prominent feature of non-human primates living in multimale-multifemale groups (e.g. Stumpf et al., 2011). Owing to the imbalance in parental investment, males and females pursue different goals, and use a variety of mating strategies in order to increase their reproductive success (Bateman, 1948; Trivers, 1972). Whereas males typically compete to monopolize and mate with as many females as possible, females should be more selective of their mates. In multimale-multifemale groups, however, females face the risk of infanticide by males, and hence have evolved a number of counterstrategies to decrease monopolization by males and to manipulate male paternity assessments (e.g. Clarke et al., 2009; van Schaik et al., 1999, 2000, 2004). Usually, females attempt to dilute paternity by mating promiscuously while at the same time biasing paternity to 'high quality' males by mating selectively (e.g. van Schaik et al., 1999, 2000, 2004). Each sex may oppose the strategies of the other sex leading to the co-evolution of counterstrategies, and raising the question which sex prevails in intersexual conflict. Assuming that males predominantly control reproduction, numerous studies have focused on male-male competition and male coercion of females (for review see Stumpf et al., 2011). More recently, researches have become more interested in the females' perspective in order to illuminate how successful females are in manipulating and choosing their mates, given that males are likely to have different interests (for review see Stumpf et al., 2011).

The present thesis was designed to investigate female reproductive strategies and the underlying proximate mechanisms in seasonally breeding Assamese macaques, and furthermore, to examine basic female reproductive physiology and life-history parameters. Recently, it has been shown that direct male-male competition in this species is low compared to closely related species (Ostner et al., 2011), suggesting that females may have a great impact on mating and reproductive outcome. Combining hormone and behavioral data, I related female sexuality and male monopolization attempts (i.e. sexual consortships) to female fertility state, which shed new light on female mating strategies. In the following general discussion, I will summarize the main findings of my studies and put them in a comparative perspective.

\section{Female reproductive physiology \& life-history characteristics}

Chapters 1 and 2 focus on the characterization of female reproductive physiology and the determination of reproductive and life-history traits, both of which mold and constrain largely female reproductive strategies (e.g. Kappeler \& Pereira, 2003). Prior to my studies, no information was available about reproductive and life-history parameters in Assamese 
macaques. Thus, my first two chapters are a fundamental basis to investigate and understand female mating strategies in this species, which is the core of my thesis.

Non-invasive hormone analysis has become indispensable in studies of reproductive and behavioral endocrinology in wild animals (e.g. Anestis, 2010; Whitten et al., 1998). For the first time in Assamese macaque females, I applied fecal hormone analyses (progestogen, estrogens, and androgens), and show that non-invasive endocrine characterization of female reproductive state is principally possible in this species. Progestogen profiles could be used reliably to assess female ovarian cyclicity, and to determine the timing of ovulation and conception. Estrogen profiles, on the contrary, were less clear and inconsistent across females. For example, a clear pre-ovulatory estrogen peak (indicating follicular maturity; e.g. Knobil \& Neill, 2006) could not be detected in all females. Estrogen measurements can therefore not be used reliably in future research on Assamese macaques as it remains unclear whether the observed variation reflects a species-specific estrogen metabolism or is due to methodological issues. In contrast, androgen measurements have been proven successful, and presumably useful. To my knowledge for the first time in a wild primate, I investigated fecal androgen excretion throughout the entire gestation period, which revealed a pattern resembling markedly the ones from blood serum studies in closely related cercopithecine species. The pronounced rise in fecal androgens during the first trimester of gestation may allow for early pregnancy detection. In addition, androgen investigations revealed fetal-sex effects on maternal androgen secretion, with females carrying male fetuses exhibiting higher fecal androgen levels in the third trimester compared to femalecarrying mothers, potentially allowing for a prenatal sex determination.

Timing of reproduction was strictly seasonal. All births occurred between April and July ( $86 \%$ in April-June), and conceptions were confined to October-February $(65 \%$ in December-January). Progestogen profiles revealed that females were anovulatory during the non-mating season and mostly conceived during their first ovulatory cycle within the mating season (mean: 1.2 cycles to conception). The strong seasonality was reflected also in the lengths of interbirth intervals (IBI). A combination of hormone data (i.e. conception dates) and birth records showed that, in Assamese macaques, the $\mid \mathrm{BI}$ is bimodally distributed, i.e. females who conceive early in the mating season can conceive again in the consecutive year (13.9 months), otherwise they will have their next infant after two years (23.2 months). Postpartum amenorrhea (PPA) for females with 1-year IBIs was relatively constant (265-290 days), and gestation length fell into the range of closely related species (164.2 days; see Kappeler \& Pereira, 2003).

Overall, my first two chapters make an important contribution to the comparative literature on reproductive and life-history parameters in primates and most importantly, build the essential information needed to study mating strategies and the mechanisms involved. 
The patterns of female reproductive physiology and life-history strongly suggest environmental constraints acting on female Assamese macaques. The short mating season during which females mostly conceive in their first ovarian cycle is, presumably, challenging for females in terms of manipulating both male monopolization potential and paternity estimates, suggesting female adaptations to circumvent physiological trade-offs.

\section{Female reproductive strategies in Assamese macaques}

In chapters 3 and 4 , I investigate female reproductive strategies and the underlying proximate mechanisms. I ascertain the patterns of female sexual activity, and determine what females reveal about their fertility status. The successful endocrine determination of the timing of ovulation/conception enabled me to relate sexual swellings, female sexual behavior, and male sexual consortships (as an indirect indicator of male knowledge about female reproductive state) in time to female fertility. Overall, these two chapters were set out to better understand the determinants of mating and reproductive outcome and the interaction between male and female reproductive strategies in a species with low male contest competition (see Ostner et al., 2011).

Females became sexually active in unison up to 3 months before the onset of ovarian cyclicity, and were receptive throughout the 4-month mating season including acyclic, cyclic, and pregnant stages. This indicates that female sexual behavior is largely decoupled from hormonal control in this species. On nearly $90 \%$ of days there was more than one female receptive. Females actively initiated $70 \%$ of all copulations, mated at low daily frequencies, and showed no signs of behavioral estrus (e.g. see Dixson, 1998), i.e. mating frequencies were not increased during fertile compared to pre- and post-fertile stages, which again suggests a minor hormonal influence on female sexual behavior. Overall, females showed no apparent behavioral signs of fertility.

Likewise, sexual swellings did not reliably indicate female fertility. Conspicuous sexual swellings are common among catarrhine primates with multimale-multifemale social organization and are found in numerous macaque species (e.g. Dixson, 1983; 1998). The functional significance of female sexual swellings has long been debated (reviewed by Zinner et al., 2004), but it is widely agreed that swellings are graded signals of ovulation probability which enable females to balance paternity concentration and dilution (Nunn, 1999b; Zinner \& Deschner, 2000). Assamese macaque females do not exhibit prominent sexual swellings. The small subcaudal swellings found in only some females showed no apparent cyclic size changes and were not obviously linked to hormonal profiles or the timing of conceptions. Thus, sexual swellings are not graded signals of ovulation probability in this species. 
Taken together, the lack of (1) behavioral estrus, (2) sexual swellings, and (3) copulation calls (pers. obs.) indicate that in Assamese macaques fertility is obscured. The imperfect male monopolization attempts by high-ranking males, i.e. long sexual consortships (up to 6 weeks) which were not linked to female fertile phases, underscore that female fertility is undisclosed to males. This finding is unusual because although in most primate species fertility is unpredictable to some extent (e.g. van Schaik et al., 2000), truly concealed fertility has, if at all, been rarely observed, and has been subject to intensive debate (e.g. Burt, 1992; Gray \& Wolfe, 1983; Strassmann, 1981; Thornhill \& Gangestad, 2008). Recently, Thornhill \& Gangestad (2008) reevaluated the concept of concealed fertility and the hypotheses that have been proposed to explain its evolution, coming to the conclusion that females could benefit from concealed fertility whenever they receive male-delivered benefits through extended sexuality.

In Assamese macaques, concealed fertility appeared to enhance extended sexuality to an extreme, suggesting important benefits associated therewith (see below). Although mostly conceiving in their first ovarian cycle, i.e. avoiding an increased number of cycles to conception, which may prolong the total length of their mating period (see Clarke et al., 2009), females mated rather continuously throughout the entire 4-month mating season resulting in a considerable amount of non-procreative mating (94\%), and leading to several females being receptive at the same time (see above). On a proximate level, the unusual extended female sexuality (beyond ovarian cyclicity) is puzzling and cannot be explained by overlapping ovarian cycles (see below), suggesting a social facilitation of mating activity (e.g. Rowell \& Hartwell, 1978; Vandenbergh \& Drickamer, 1974).

In chapter 4, I investigate patterns of female reproductive synchrony. It is widely acknowledged that the spatiotemporal distribution of receptive females affects largely male mating opportunities, and that females can become simultaneously receptive by synchronizing their ovarian cycles (e.g. Cowlishaw \& Dunbar, 1991; Emlen \& Oring, 1977; Kutsukake \& Nunn, 2006; Ridley, 1986). In non-human primates, however, evidence for ovarian cycle synchrony is rare and inconclusive (e.g. Homo sapiens: McClintock, 1971; Strassman, 1997; Pan troglodytes: Wallis, 1985; Matsumoto-Oda et al., 2007; Leonthopithecus rosalia: French \& Stribley, 1987; Monfort et al., 1996; Papio hamadryas: Zinner et al., 1994; Tobler et al., 2010; Mandrillus sphinx: Setchell et al., 2010a; Lemur catta: Pereira, 1991). I propose an alternative hypothesis, the mating-synchrony-hypothesis, i.e. that females synchronize their mating behavior and not their ovarian cycles, which is confirmed by the results of this study. As such, the occurrence of a female's receptivity was significantly associated with the proportion of other females mating on a given day. Female fertile phases, on the contrary, were randomly distributed, and females who spent more time in close proximity did not cycle more closely together (pheromone hypothesis; McClintock, 
1984; Stern \& McClintock, 1998). The behaviorally coordinated mating activity (i) underscores the emancipation of sexual behavior from hormonal control, (ii) affects and increases considerably the number of simultaneously receptive females, and (iii) offers a potential proximate explanation for the patterning and maintenance of extended female sexuality.

The extremely prolonged sexual activity appeared to enable females to efficiently exercise their mating strategy. Females showed a high degree of promiscuity, i.e. they copulated with virtually all males. Dominant males did not monopolize fertile matings, and females mated with several males during their fertile periods. In contrast to numerous nonhuman primate species (e.g. van Schaik et al., 1999, 2000, 2004), females did not concentrate paternities to high-ranking males but expressed significant non-dominance based mating biases, i.e. they mated repeatedly with a 'primary partner' who obtained on average $25 \%$ of a female's total number of copulations. Most notably, females preferred different males including high- and low-ranking, adult and large subadult males. These mating biases suggest a strategy to obtain male delivered benefits, e.g. protection, friendships, and/or care for future infants (Assamese macaque males frequently interact and care for infants; pers. obs.), which are yet to be investigated (see Hrdy, 1979, 2000; Rodríguez-Gironés \& Enquist, 2001; Smuts, 1985; Soltis, 2002; Thornhill \& Gangestad, 2008).

In summary, I have shown that female fertility is concealed in Assamese macaques, and that females exhibit remarkable behavioral adaptations, i.e. extreme extended female sexuality and active mating synchrony. These traits increase the number of simultaneously receptive females; hence considerably reduce male monopolization potential. Obviously, this raises the questions how and to what extent these rather unusual traits affect mating and reproductive outcome, and male (and the co-evolution of male and female) reproductive strategies.

\section{Intersexual conflict in Assamese macaques}

Intersexual conflict in primates living in multimale-multifemale groups is inevitable (e.g. Stumpf et al., 2011). Infanticide by males is an intense form of male sexual conflict and creates a 'female dilemma' (e.g. van Schaik et al., 1999, 2000). Generally, females should respond to the threat of infanticide by mating promiscuously and thereby diluting paternities among multiple males, while at the same time concentrating paternity into high-quality (dominant) males to promote infant protection (e.g. van Schaik et al., 1999, 2000). Concurrently, however, males will attempt to monopolize matings, which leads to the evolution of female traits to decrease male monopolization potential and so on. Sexually 
antagonistic co-evolution, i.e. an adaption in one sex that promotes a counter-adaption in the other sex, hence leading to an 'arms race' between males and females, occurs in virtually all sexually reproducing species (e.g. Arnquist \& Rowe, 2005). But which sex prevails in sexual conflict? Assessing this question is challenging and requires thorough knowledge of male and female reproductive strategies and the underlying proximate mechanisms as they may reflect the severity of intersexual conflict (e.g. Clarke et al., 2009; Pradhan \& van Schaik, 2008; Stumpf et al., 2011; van Schaik et al., 2004).

As shown recently, male Assamese macaques exhibit the lowest alpha male mating skew when compared to other macaque species (17.5\%; Ostner et al., 2011), despite a clear male dominance hierarchy (Ostner et al., 2008b, 2011). Also, alpha male paternity is relatively low (33\%; Schülke et al., 2010). Thus, females appear to manipulate and control largely mating and reproductive outcome in this species. Females show no cues to fertility, and exhibit extreme extended sexuality and active mating synchrony (see above) all of which reduce male-male competition for mates. But how do males react to this low-contest situation? In response to their low monopolization potential and although mate guarding may be costly (e.g. Alberts et al., 1996; Weingrill et al., 2003), dominant males attempt to monopolize matings in that they form long sexual consortships (up to 6 weeks) with some females (Chapter 3; Ostner et al., 2011). These consortships are far from perfect as they are not linked to female fertility (Chapter 3), and hence seem to reflect a "best-of-a-bad-jobstrategy" by high-ranking males (Ostner et al., 2011).

Perhaps, in turn, the extreme extended female sexuality, at least partly, is molded by these lengthy male monopolization attempts. It remains unclear how sexual consortships are formed in this species, i.e. whether they are a sole form of male sexual coercion (see Smuts \& Smuts, 1993; Muller \& Wrangham, 2009) or whether they entail also benefits for females. During the long consorts, males and females stay in close proximity, groom, mate, and coordinate their movements. Males mostly follow their partner, and females appear to 'wait' for the male (pers. obs.), suggesting also female benefits. However, by being 'cooperative' females may simply avoid male aggression/coercion which, however, is rare in Assamese macaques (see below). If females have no bearing on consortship formation (and termination), i.e. if they happen to be consorted for a considerable amount of time at any point during the 4-month mating season, it may pay to mate throughout the entire period. Consortships, although not entirely exclusive, limit substantially the females' number of mating partners while being consorted (Chapter 3), and thus, may constrain females in exerting their strategies, i.e. achieving a high degree of promiscuity and creating differentiated mating relationships.

In chapter 3, I show that females express non-dominance based mating biases towards different males. One could argue that these differentiated mating relationships reflect 
male mating tactics rather than female preferences, i.e. 'female choice' in response to male sexual coercion (e.g. Muller et al., 2011). If this was the case in Assamese macaques, however, one would expect male rank effects, i.e. observed female preferences following the priority of access model (POA; see Altmann, 1962). The females' 'primary' partners, however, include males of all ranks (including large subadult, low ranking males). Furthermore, males rarely direct aggression towards females (pers. obs.), forced copulations are virtually absent (pers. obs.), and females actively initiate the majority of copulations (70\%; Chapter 3), altogether indicating the active role of females.

Daily mating frequencies are low, which may be partly male-driven. Although $94 \%$ of copulations occur during female non-fertile stages, Assamese macaque males exhibit high rates of ejaculatory copulations (nearly $90 \%$; Chapter 3 ) which presumably reflect their lacking knowledge about female fertility state. Ejaculate production and ejaculatory copulations are costly (Dewsbury, 1982; Thomson et al., 2006), and sperm number decreases with frequent ejaculatory copulations (Marson et al., 1981; Preston et al., 2001). Thus, in order to "adjust" to the females' extreme extended female sexuality, males may decrease mating frequencies (which may also illuminate the high rates of copulation initiations by females). Vervet monkeys (Cercopithecus aethiops), for example, exhibit a very similar mating pattern with extended female sexuality and low mating frequencies (Andelman, 1987) supporting the suggested negative relationship between the length of the overall mating period and copulation frequencies.

Taken together, my studies have contributed to a better understanding of the interaction between male and female mating strategies in Assamese macaques, and illuminate the causes of the low male reproductive skew in this species. But why do both female sexuality and the overall mating (and consortship) pattern differ obviously from most other, closely related species? Why do female Assamese macaques not concentrate paternity into the dominant male but provide different males with high paternity estimates? Recently, van Noordwijk \& van Schaik (2004) pointed out that "the low end of the paternity concentration spectrum is least understood". So, can this low-skew species possibly provide new insight into sexual conflict and the determinants of paternity concentration in multimalemultifemale primate groups?

\section{Paternity concentration in multimale-multifemale primate groups: comparative \& evolutionary reflections}

Paternity concentration generally depends on the dominant male's potential to monopolize (fertile) females which is affected by (i) female preferences, (ii) the number of males, and (iii) the number of reproductively active (i.e. simultaneously receptive) females in a group 
(reviewed by van Noordwijk \& van Schaik, 2004; Nunn, 1999a; Ostner et al., 2008a; see Fig. 1). The latter is affected by the degree of seasonality of the species in question, i.e. in seasonal breeders more females will become receptive at the same time (e.g. Cowlishaw \& Dunbar 1991; Paul, 1997; Ridley 1986). In line with this, a strong negative relationship between seasonality and paternity concentration in the top-dominant male has been found (van Noordwijk \& van Schaik, 2004). Furthermore, the source of dominance acquisition affects (and is affected by) female preferences and hence paternity concentration in the alpha-male, i.e. in inside take-over species concentration in the dominant male is less important compared to outside take-over species (Pradhan \& van Schaik, 2008; see also van Noordwijk \& van Schaik, 2004).

Based on my finding that females 'concentrate' in different males, I suggest that the traditional view that females should prefer the dominant male (assuming him to be the one best placed to protect or care for infants; see above) may not hold for all species. I propose an extension of the above described conceptual model, and introduce a link between seasonality and female preferences (Fig. 1). Seasonality may decrease the risk of infanticide (e.g. Hrdy, 1979; Hrdy \& Hausfater, 1984), however, in species with longer than 1-year interbirth intervals, infanticide may shorten them, hence be a favorable male strategy (e.g. Borries, 1997; Soltis et al., 2002; see also van Schaik, 2000a). In highly seasonally breeding species with many females, all infants are born during a short period of time (over $67 \%$ in three months; van Schaik et al., 1999) and thus, in the case of male immigration and/or takeover of the alpha male position, they will all be exposed to the new male at the same time. In other words, the number of infants who are simultaneously at high risk of infanticide is higher in seasonal compared to non-seasonal species. In this case, the dominant male may not be able to protect all of them at the same time, and thus, females will benefit from recruiting and 'concentrating' in different protectors for their infants (Fig. 1). Conversely, in non-seasonal species, infants are born throughout the year, i.e. at any given point in time only few infants will be vulnerable to infanticide, and thus, the dominant male (Fig. 1) may be capable of efficiently protecting them. In this case, preferring and concentrating paternity in the dominant male may be beneficial for females. Similarly, in case of a predation event females in seasonal species may benefit from different infant protectors.

Assuming this dichotomy between seasonal and aseasonal species, the potential female mechanisms used to manipulate male monopolization potential, i.e. to express their 'preferences', need to be incorporated (Fig. 1) and reevaluated as they may vary among the two groups. Non-human primate females in multimale-multifemale groups have evolved a number of mechanisms against male coercion and infanticide which are assumed to enable females to (i) manipulate the distribution of paternity among a pool of males and (ii) manipulate the information available to males upon which to base their paternity estimates 
van Schaik, 2000b, van Schaik et al., 1999, 2004). Male primates usually cannot directly recognize their infants (but see Buchan et al., 2003), hence must rely on their mating history with a given female, i.e. copulation frequencies in relation to female attractivity (e.g. Soltis et al., 2000; van Schaik et al., 2004). In the following section, I will discuss the significance of potential female mechanisms in more detail.

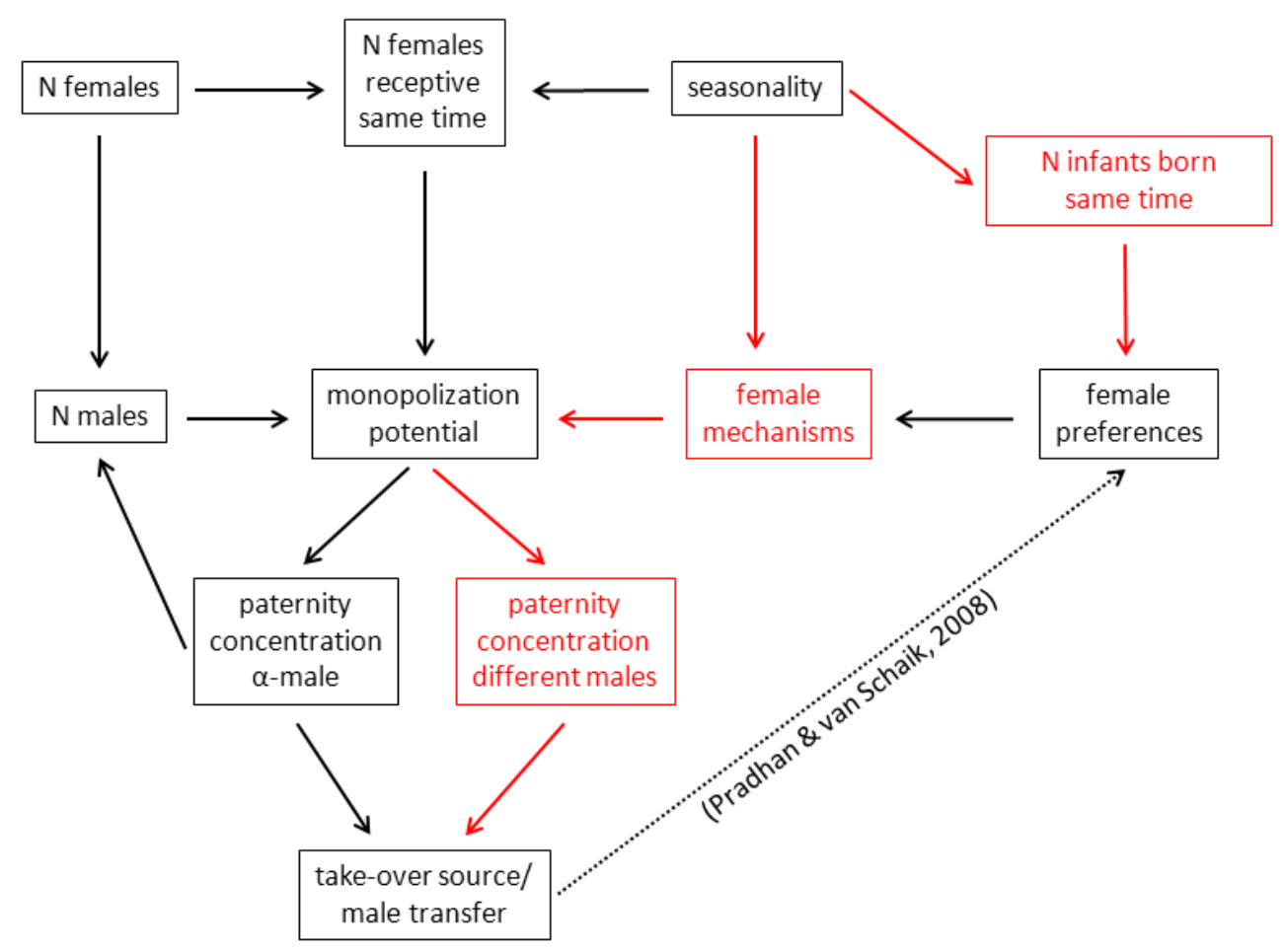

Figure 1: Predicted mechanisms of paternity concentration in catarrhine primates with multimale-multifemale social organization and female philopatry. Flowchart extended and modified (red boxes and arrows) after van Noordwijk \& van Schaik (2004). See text for details.

\section{Female mechanisms affecting male monopolization potential}

The interspecific variation and combination of female mechanisms used to manipulate male monopolization potential are puzzling and have long attracted researchers' interest (e.g. Clarke et al., 2009; van Schaik et al., 1999, 2000, 2004). Generally, it is assumed that female mechanisms are unequally costly, i.e. behavioral alterations are cheap and physiological traits are more expensive, and hence the latter should be found in species where the efficacy of male coercion (i.e. monopolization potential) is high (Clarke et al., 2009; van Schaik et al., 1999). Seasonal species are assumed to have more behavioral freedom, hence do not need expensive traits (e.g. conspicuous sexual swellings, increased number of cycles to 
conception; Clarke et al., 2009; van Schaik et al., 1999, 2004). According to their unequal costs, Clarke et al. (2009) suggest the following order in which female counterstrategies should appear: active mate selection, sneaky copulations, copulation calls, longer mating periods, sexual swellings, and an increased number of ovarian cycles. Active promiscuity and sneaky copulations undoubtedly are a general phenomenon, however, across primates we observe great variation in the lengths of mating periods, number of cycles to conception, and most notably in the occurrence of sexual signals, i.e. copulation calls and sexual swellings (e.g. Clarke et al., 2009; Maestripieri \& Roney, 2005; van Noordwijk \& van Schaik, 2000; van Schaik et al., 1999, 2004). This variation, however, may not necessarily be linked to different energetic costs but may also reflect divergent female needs (see above). I will address (i) sexual swellings and (ii) the number of cycles to conception in more detail as follows.

Sexual swellings are found in numerous, yet not all, catarrhine primates (see van Schaik et al., 1999; Fig. 8.3). Extensive research has been conducted on species exhibiting these sexual signals in order to explain their functional significance (see Zinner et al., 2004 for a review). Among several hypotheses, the most widely acknowledged is the graded-signal hypothesis (Nunn, 1999b; Zinner \& Deschner, 2000) which combines the reliable-indicator hypothesis (females compete for males and signal their reproductive quality through sexual swellings; Pagel, 1994), the best-male hypothesis (swellings incite male-male competition, thus increasing the chances to acquire desirable traits; Clutton-Brock \& Harvey, 1979; Hrdy, 1979), and the many-males hypothesis (swellings enable females to mate promiscuously in order to dilute paternity among males; Hrdy, 1981; Hrdy \& Whitten, 1987). Nunn (1999b) argues that sexual swellings function to attract the dominant male when ovulation probability is highest while also attracting lower-ranking males when ovulation probability is low but nonzero, hence enabling females to balance paternity concentration and dilution. In other words, in species that possess these signals, all females concentrate in the same male (i.e. the dominant male) who will monopolize them at peak swelling. Conversely, in species without swellings females can still provide males with different paternity estimates but they can do so in any male irrespective of male rank, and most importantly, each female can raise the paternity estimates for a different male (Chapter 3 ).

In evolutionary terms, if swellings (or other cues to fertility) lead to a concentration in one (dominant) male, and if in seasonal species this single male is not capable of protecting (or care for) all infants (see above), some infants may be disadvantaged and may not survive, hence affecting female (and male) lifetime reproductive success. In this case, swellings would be selected against. Thus, the evolutionary loss of sexual swellings in certain Old World primates may be explained by reduced reproductive rather than energetic costs (Clarke et al., 2009; see above). Most seasonal species with multimale-multifemale social organization lack prominent sexual swellings (Dixson, 1983, 1998; van Schaik et al., 1999) 
supporting my assumption that in seasonal species females do not aim for paternity concentration in the dominant male.

Similar to sexual swellings, an increased number of cycles to conception may considerably affect female reproductive success, in particular in seasonal species. For example, female Assamese macaques who conceive early in the mating season can conceive their next infant in the consecutive year, whereas females who conceive late will have their next infant after two years (Chapter 1). A similar relationship between early conceptions and shorter interbirth intervals has been reported for captive bonnet macaques (Macaca radiata; Silk, 1990). Thus, it seems plausible that in seasonal species females mostly conceive in their first ovarian cycle (e.g. Fürtbauer et al., 2010; Küster \& Paul, 1984; Lindburg, 1971; Möhle et al., 2005; Takahata, 1980). In aseasonal species, on the contrary, an increased number of cycles to conception may prolong interbirth intervals for only a few months, and thus, may be reproductively less costly.

Overall, the variety in the occurrence of female mechanisms may be explained by (i) divergent female preferences (i.e. dominant versus different males) and (ii) reproductive rather than energetic burdens. But what are the mechanisms females 'need' in order to distribute high paternity estimates among different males? In species with concentration in the dominant male, females, in general, may 'adjust' male monopolization potential in both possible directions (i.e. increase versus decrease), advertise fertility to some extent and balance paternity concentration and dilution. Conversely, in species with paternity concentration in different males, females should attempt to (1) decrease the dominant male's monopolization potential towards zero, i.e. avoid all traits advertising fertility, and (2) employ mechanisms which allow them to create differentiated mating relationships (this study). The only estimates of paternity probability males in such species may use, are the number of matings (males cannot relate matings to fertility because it is undisclosed). Thus, in order to provide males with different paternity estimates females need to copulate more frequently with their preferred male compared to other males in the group, i.e. matings become probabilistic cues of paternity certainty (Chapter 3 ).

Paternity dilution, i.e. mating with multiple males and providing them with a non-zero probability of paternity may be relatively easy to achieve in such species, whereas creating differentiated mating relationships and mating repeatedly with a 'primary' partner may require a long mating period. Socially facilitated coordination of female sexuality, i.e. an extreme form of emancipation of sexual behavior from hormonal control, can considerably prolong mating periods, hence may be beneficial in species where females conceive in their first cycle (Chapter 4). Active mating synchrony affects largely male monopolization potential, and thus needs to be added to the 'list' of female sexual counterstrategies. Behavioral coordination of copulatory behavior, however, may not be restricted to seasonal species, and 
generally may be considered beneficial if concentration in the dominant male is less important (e.g. inside take-over species; see Pradhan \& van Schaik).

\section{Conclusions}

The findings from my studies make several contributions to the current literature. First, this thesis has filled an important gap in the comparative literature on female primate reproductive physiology and life-history traits. This knowledge will serve also as a fundamental base for future studies on this species. Second, my investigations and results on female reproductive strategies and the underlying proximate mechanisms add substantially to our understanding of the plasticity of female sexuality in primates. Third, this thesis addresses a species with low male reproductive skew, the least understood end of the paternity concentration continuum, and thus makes a major contribution to primate socioecology. Fourth, the study has gone some way towards enhancing our understanding and refining our concept of intersexual conflict over paternity in primates. Clearly, additional research is needed.

\section{Future directions}

This thesis not only shed new light on female reproductive strategies and the plasticity of female sexuality but also raised new questions and directions for future research on both Assamese macaques and other non-human primate species, which I summarize as follows:

(1) Investigating male-female associations, both outside the mating season and in a longterm perspective (e.g. throughout consecutive mating seasons), will help to illuminate female mating preferences during the mating season. The differentiated mating relationships may, for example, translate into male-female 'friendships' during lactation, and may further also predict the probability to sire future offspring (e.g. baboons: Smuts, 1985).

(2) In line with this, as male Assamese macaques frequently interact and care for infants (Ostner \& Schülke, unpub. data, pers. obs.), studying male-infant relationships may be rewarding as they may reflect female mating preferences, and thus provide insight into the functional significance of female reproductive strategies.

(3) Paternity analyses will be crucial to assess whether preferred males are genetic fathers and whether paternities reflect a female mating strategy or are more likely a result of postcopulatory mechanisms, e.g. sperm competition or cryptic female choice (e.g. Eberhard, 1996; Yasui, 1997). Furthermore, genetic studies could clarify 
whether females prefer MHC-dissimilar males as seen in other species (e.g. Schwensow et al., 2008; Setchell et al., 2010b).

(4) Given the strict reproductive seasonality of Assamese macaques, investigations of ecological factors, i.e. climate, food availability, and the quality of the diet, are needed in order to understand better the environmental constraints acting on females in this population. Variation in food availability and nutritional quality may further have a drastic impact on female reproduction and life-history because malnutrition may cause metabolic stress, hence affecting female energy status, stress load and reproductive function. Using an integrative approach that combines female reproduction and physiology with ecology could help to disentangle the factors shaping reproductive strategies in Assamese macaques.

(5) Examining both the potential costs of mate guarding (males) and being mate guarded (females), e.g. by monitoring physical condition and glucocorticoid levels (as an indicator of stress), may elucidate what factors drive the patterning of long sexual consortships in Assamese macaques, and furthermore, may help to evaluate whether they are solely a form of male sexual coercion or rather mutual male-female associations.

(6) Comparative studies on closely related species without overt cues to fertility (i.e. species where females lack prominent sexual swellings and copulation calls, e.g. macaque species belonging to the sinica-arctoides lineage; see Maestripieri \& Roney, 2005) may contribute towards a better understanding of the evolution of the rather unusual female mating patterns in Assamese macaques. Furthermore, testing the mating-synchrony-hypothesis in other species (e.g. species with a higher observed than expected mating overlap; see Nunn et al., 2001) would help to assess its general applicability. 



\section{SUMMARY}

Sexual conflict is a prominent feature of animals living in multimale-multifemale groups, and drives the evolution of sex-specific reproductive strategies. Owing to divergent reproductive optima, males should compete to monopolize and mate with as many females as possible, whereas females should be more selective of their mates, leading to a coevolutionary arms race between the sexes. Facing the risk of infanticide by males, a source of intense sexual conflict, female primates have evolved counterstrategies and mechanisms to decrease male monopolization potential (i.e. male mating skew) and to manipulate male paternity estimates to overcome the 'female dilemma', i.e. to balance paternity concentration and dilution.

The aim of this thesis was to investigate female reproductive strategies and underlying proximate mechanisms and constraints in a primate with low male contest competition, the Assamese macaque (Macaca assamensis). By combining demographic, behavioral and hormone data, I investigated general female reproductive parameters and life-history traits, and in addition, I visually monitored anogenital sexual swellings in order to investigate their functional significance. I examined female sexual activity and male consortships in relation to fertility, and investigated patterns of reproductive synchrony. I proposed and tested the hypothesis that females behaviorally coordinate their mating activity, and investigated also ovarian cycle synchrony. The study was conducted at Phu Khieo Wildlife Sanctuary in northeastern Thailand during two consecutive mating seasons (07/08 and 08/09). I collected focal animal (>800 hours) and ad libitum data from 16 Assamese macaque females living in a wild, fully habituated group consisting of 50-60 individuals. Furthermore, I collected more than 2300 fecal samples in order to describe ovarian cycle and pregnancy characteristics and to assess the timing of ovulations and conceptions using enzyme immune assays (EIA) for estrogen, progestogen and androgen metabolites.

Endocrine profiles revealed that non-invasive monitoring of female reproductive state is feasible in Assamese macaques. Patterns of fecal androgen excretion during gestation may allow for early pregnancy detection and prenatal sex determination. Gestation length and interbirth interval fell into the range of closely related species. Females were anovulatory during the non-mating season and mostly conceived during their first ovulatory cycle within the 4-month mating season, with $65 \%$ of conceptions confined to December and January. The small subcaudal sexual swellings did not signal ovulation probability, and females lacked copulation calls and signs of behavioral estrus all of which indicate that fertility is undisclosed to males in this species. Accordingly, the long sexual consortships by high-ranking males were not linked to female fertile phases. Females exhibited extreme extended sexuality and mated throughout the mating season including acyclic and pregnant stages. Female fertile phases were randomly distributed, but the occurrence of a female's receptivity was significantly associated with the proportion of other females mating on a given day, offering a 
potential proximate explanation for the patterning and maintenance of extended sexuality in this and presumably other species. In addition, and in contrast to numerous non-human primate species, females did not concentrate paternities to high-ranking males but expressed non-dominance based mating biases, i.e. they mated repeatedly with a 'primary partner' despite exhibiting high rates of promiscuity.

Overall, these results suggest that in response to constraints of reproductive and lifehistory traits, female Assamese macaques modify and emancipate their sexual behavior from hormonal control, and thereby manipulate and control largely mating and reproductive outcome. In conclusion, this $\mathrm{PhD}$-project not only gathered initial information on reproductive parameters and life-history traits for the seasonally breeding and rarely studied Assamese macaque but also contributes to the growing body of knowledge that endeavors to understand reproductive strategies of female vertebrates, sheds new light on the plasticity of female sexual behavior, and contributes to a refinement of our concept of intersexual conflict over paternity in primates. 


\section{ZUSAMMENFASSUNG}

Sexueller Konflikt ist ein typisches Merkmal von Tieren in gemischten Geschlechtergruppen, und treibt die Evolution von geschlechtsspezifischen Reproduktionsstrategien. Aufgrund divergierender reproduktiver Optima, sollten Männchen untereinander konkurrieren, um möglichst viele Weibchen zu monopolisieren und sich mit innen zu paaren. Im Gegensatz dazu sollten Weibchen ihre Partner selektiv auswählen. Dies führt zu einem koevolutionären Wettstreit zwischen den Geschlechtern. Das potentielle Risiko von Infantizid durch Männchen kreiert ein Dilemma für Weibchen. Um das Infantizidrisiko zu verringern und die Vaterschaft gleichzeitig zu konzentrieren und zu verschleiern, entwickeln Weibchen Gegenstrategien und Mechanismen, die das Monopolisierungspotential der Männchen (d.h. den male mating-skew) herabsetzen und deren Abschätzungen der Vaterschaftswahrscheinlichkeit manipulieren.

Das Ziel dieser Dissertation war es, weibliche Reproduktionsstrategien und deren zugrundeliegende proximate Mechanismen eines Primaten mit geringer direkter Konkurrenz unter Männchen, dem Assammakaken (Macaca assamensis), zu untersuchen. Durch eine Kombination von Verhaltensbeobachtungen, demographischen, und Hormondaten, konnten generelle weibliche Reproduktionsparameter und life-history Merkmale untersucht werden. Zusätzlich wurden Größenveränderungen in Anogenitalschwellungen dokumentiert, um deren funktionelle Bedeutung zu bestimmen. Die sexuelle Aktivität der Weibchen und ihre consortships mit Männchen wurden in Relation zur Fertilität gesetzt. Desweiteren wurden die zeitliche Verteilung und Überlappung von fertilen Phasen untersucht, und eine neue Hypothese, dass Weibchen ihre Paarungsaktivität unabhängig von hormonellem Einfluss synchronisieren, aufgestellt und getestet. Die Studie wurde in zwei aufeinanderfolgenden Paarungszeiten (07/08 und 08/09) im Phu Khieo Wildlife Sanctuary im Nordosten Thailands durchgeführt. Von 16 Weibchen einer freilebenden, habituierten Gruppe von 50-60 Individuen wurden Fokustier- (>800 Stunden) und ad libitum-Daten erhoben. Darüber hinaus wurden über 2300 Kotproben gesammelt, um mit Hilfe von Enzym-Immuno-Assays (EIA) für Östrogen-, Gestagen- und Androgenmetabolite Zyklus- und Schwangerschaftscharakteristika $z u$ beschreiben und Ovulationen und Konzeptionen zu bestimmen.

Die endokrinen Profile zeigten, dass es möglich ist, den Reproduktionsstatus von Assammakakenweibchen mittels non-invasiver Methoden zu charakterisieren. Die Muster fäkaler Ausscheidung von Androgenen erlauben eine frühe Schwangerschaftserkennung und eine pränatale Geschlechtsbestimmung des Fötus. Die Tragzeit und das Intergeburtenintervall fielen in den Bereich nahe verwandter Arten. Außerhalb der Paarungszeit waren Weibchen anovulatorisch und konzipierten meist im ersten Ovulationszyklus innerhalb der 4-monatigen Paarungszeit. 65\% aller Konzeptionen fanden im Dezember und Januar statt. Die unauffälligen subkaudalen Sexualschwellungen 
signalisierten nicht die Ovulationswahrscheinlichkeit, und die Weibchen zeigten keine Kopulationsrufe oder Anzeichen von Verhaltensöstrus. Dies weist auf eine verschleierte Fertilität bei Weibchen dieser Art hin. Demgemäß standen die langen consortships mit hochrangigen Männchen in keinem direkten Zusammenhang mit den fertilen Phasen. Die Weibchen waren über die gesamte Paarungszeit sexuell aktiv und kopulierten auch in azyklischen und trächtigen Phasen. Die fertilen Phasen der Weibchen waren zufällig verteilt. Das Auftreten weiblicher Rezeptivität war jedoch signifikant mit dem Anteil anderer sich paarender Weibchen assoziiert. Dies stützt die mating-synchrony-hypothesis und bietet eine mögliche proximate Erklärung für das ausgeprägte, nicht reproduktive Sexualverhalten bei dieser und vermutlich auch bei anderen Arten. Im Gegensatz zu zahlreichen Primatenarten, konzentrierten die Weibchen Vaterschaften nicht in hochrangige Männchen, sondern zeigten Paarungspräferenzen für Männchen unterschiedlichsten Ranges. Trotz ausgeprägter Promiskuität, paarten sich Weibchen wiederholt mit ihrem jeweiligen „primären Partner“.

Die Ergebnisse meiner Studie haben insgesamt gezeigt, dass AssammakakenWeibchen ihr Sexualverhalten adaptieren und von strikter hormoneller Kontrolle lösen. Dadurch können Weibchen den Paarungs- und Reproduktionserfolg der Männchen zu einem großen Teil manipulieren und kontrollieren. Diese Dissertation hat (1) erste Informationen über Reproduktionsparameter und life-history Merkmale bei dieser saisonalen und kaum erforschten Makaken-Spezies geliefert, (2) den wachsenden Wissensstand über Reproduktionsstrategien weiblicher Wirbeltiere erweitert, (3) neues Licht auf die Plastizität weiblicher Sexualität geworfen, und (4) zu einer Verfeinerung unseres Konzepts von intersexuellem Konflikt bezüglich Vaterschaft bei Primaten beigetragen. 


\section{ACKNOWLEDGMENTS}

During my studies many people from different countries and cultures have helped, and who I wish to thank at this point. I wish to thank the National Research Council of Thailand (NRCT) and the Department of National Parks, Wildlife and Plant Conservation (DNP) for permission to conduct research in Thailand. I am also grateful for funding from the Max Planck Society, the National Geographic Society, and the German Initiative of Excellence.

I am much obliged to my advisors and collaborators. In particular, I wish to thank Julia Ostner and Oliver Schülke for providing me the opportunity to work at Phu Khieo, for excellent supervision and constructive comments on the manuscripts, for continuous multifaceted support during the course of this $\mathrm{PhD}$, and for treating me as a scientist rather than just a student. I am also grateful to Michael Heistermann for his great collaboration. Michael provided valuable input on the manuscripts, and he was always open for discussions on reproductive physiology. I wish to thank Roger Mundry for sharing his stats expertise and collaboration in chapter 4. I would also like to thank Peter Kappeler for his support as a referee for this dissertation.

The fieldwork in Thailand would not have been possible without the support and assistance from many people. Many thanks go to Carola Borries and Andreas Koenig who developed the field site, and to J. Prabnasuk, K. Nitaya, K. Kumsuk and K. Kreetiyutanont for their cooperation and permission to carry out this project. My deep thanks go to all the kind and welcoming people in Thung Ka Mang. I would especially wish to thank Ba Thong for her delicious Thai cuisine, Khun San and Khun Krya for washing damp, muddy, sweaty, tick covered field clothes, and Khun Kitti for his valuable help with batteries, generators, freezers, motorbikes, cars, hot showers, lights, machetes, and lastly, of course, for many hilarious evenings.

I am very grateful to "Team Macaque" with all its members. In particular, I wish to thank Miranda for improving the fieldwork in various ways, for example, and just to mention a few, for maintaining the generators, for safe motorbike rides, for perfectly dealing with complicated work plans and off day wishes, for sharing Beer Chang on the porch, for joining my off day trips to Khon Kaen, and also for plenty of discussions about monkeys and humans. Many thanks go to Khun Mee, Khun Piya, Khun Siyan, Khun Tam and Khun Wet for being great fun, for excellent teamwork, for collecting data and loads of fecal samples, and last but not least for making me feel home in Thung Ka Mang. I also thank Sebastian for collecting data. 
I thank Giwi for her endless hospitality, for introducing me to Thai history, culture, language and food (never again will I eat jellyfish $(-)$ ), and for showing me places of Thailand which I may not have ever seen without her. I would also like to thank Zach for the great time we had in Thailand.

Back in Europe, I spent hours and hours in the hormone lab which I always considered the perfect mixture of concentrated working, great know-how, and fun. I wish to thank the technicians Petra, Jutta, and especially Andrea for advice, diluting QC'S, enzyme labels and antibodies, coating plates, and preparing various solutions necessary for hormone analyses.

Last but not least, I would like to thank my family and all my friends and colleagues. I especially wish to thank my mother for her persistent and invaluable support. Many thanks go to Annie, Constance, and Mathias. I wish to thank Julitta for always being there and for visiting and travelling with me in Thailand. My deepest thanks go to Johannes for (mostly) dealing patiently with his workaholic-headstrong-girlfriend. 


\section{BIBLIOGRAPHY}

Alberts, S., Altmann, J., \& Wilson, M. (1996). Mate guarding constrains foraging activity of male baboons. Anim. Behav. 51, 1269-1277.

Altmann, S. A. (1962). A field study of the sociobiology of rhesus monkeys, Macaca mulatta. Ann. N. Y. Acad. Sci. 102, 338-435.

Altmann, J., Altmann, S. A., Hausfater, G., \& McCuskey, S. A. (1977). Life history of yellow baboons: physical development, reproductive parameters, and infant mortality. Primates. 18, 315-330.

Altmann, J., Lynch, J. W., Nguyen, N., Alberts, S. C., \& Gesquiere, L. R. (2004). Life-history correlates of steroid concentrations in wild peripartum baboons. Am. J. Primatol. 64, 95-106.

Andelman, S. J. (1987). Evolution of concealed ovulation in Vervet monkeys (Cercopithecus aethiops). Am. Nat. 12, 785-799.

Andelman, S. J., Else, J. G., Hearn, J. P., \& Hodges, J. K. (1985). The non-invasive monitoring or reproductive events in wild vervet monkeys (Cercopithecus aethiops) using urinary pregnanediol-3a-glucuronide and its correlation with behavioural observations. J. Zool. (Lond.). 205, 467-477.

Anderson, C. M., \& Bielert, C. F. (1994). Adolescent exaggeration in female catarrhine Primates. Primates. 35, 283-300.

Andersson, M. B. (1994). Sexual Selection. Princeton University Press, Princeton, NJ.

Anestis, S. F. (2010). Hormones and social behavior in primates. Evol. Anthropol. 19, 66-78.

Arnqvist, G., \& Nilsson, T. (2000). The evolution of polyandry: multiple mating and female fitness in insects. Anim. Behav. 60, 145-164.

Arnquist, G., \& Rowe, L. (2005). Sexual conflict. Princeton University press, Princeton.

Baayen, R. H. (2008). Analyzing Linguistic Data. Cambridge University Press. Cambridge.

Barelli, C., Heistermann, M., Boesch, C., \& Reichard, U. H. (2007). Sexual swellings in wild white-handed gibbon females (Hylobates lar) indicate the probability of ovulation. Horm. Behav. 51, 221-230.

Bateman, A. J. (1948). Intra-sexual selection in Drosophila. Heredity. 2, 349-368.

Bates, D., \& Maechler, M. (2010). Ime4: Linear mixed-effects models using S4 classes. R package version 0.999375-35.

Baum, M. J. (1983). Hormonal modulation of sexuality in female primates. BioScience. 33, 578-582.

Baum, M. J., Everitt, B. J., Herbert, J., \& Keverne, E. B. (1977). Hormonal basis of proceptivity and receptivity in female primates. Arch. Sex. Behav. 6, 173-192.

Beach, F. A. (1976). Sexual attractivity, proceptivity and receptivity in female mammals. Horm. Behav. 7, 105-138. 
Beehner, J. C., Phillips-Conroy, J. E., \& Whitten, P. L. (2005). Female testosterone, dominance rank, and aggression in an Ethiopian population of hybrid baboons. Am. J. Primatol. 67, 101-119.

Behboodi, E., Katz, D. F., Samuels, S. J., Tell, L., Hendrickx, A. G., \& Lasley, B. L. (1991). The use of a urinary estrone conjugate assay for detection of optimal mating time in the cynomologus macaque (Macaca fascicularis). J. Med. Primatol. 20, 229-234.

Bercovitch, F. B. (1987). Female weight and reproductive condition in a population of olive baboons (Papio anubis). Am. J. Primatol. 12, 189-195.

Bernstein, I. S., \& Cooper, M. A. (1999). Dominance in Assamese macaques (Macaca assamensis). Am. J. Primatol. 48, 283-289.

Birkhead, T. R., \& Møller, A. P. (1992). Sperm competition in birds. London: Academic press.

Boesch, C., \& Boesch-Achermann, H. (2000). The Chimpanzees of the Tai Forest: Behavioural Ecology and Evolution. Oxford University Press, Oxford.

Bolker, B. M., Brooks, M. E., Clark, C. J., Geange, S. W., Poulsen, J. R., Stevens, M. H. H., \& White, J.-S. S. (2008). Generalized linear mixed models: a practical guide for ecology and and evolution. Trends Ecol. Evol. 24, 127-135.

Borries, C. (1997). Infanticide in seasonally breeding multimale groups of Hanuman langurs (Presbytis entellus) in Ramnagar (South Nepal). Behav. Ecol. Sociobiol. 41, 139-150.

Borries, C., Launhardt, K., Epplen, C., Epplen, J. T., \& Winkler, P. (1999). Males as infant protectors in Hanuman langurs (Presbytis entellus) living in multimale groups: defence, paternity and sexual behaviour. Behav. Ecol. Sociobiol. 46, 350-356.

Borries, C., Koenig A., \& Winkler P. (2001). Variation of life history traits and mating patterns in female langur monkeys (Semnopithecus entellus). Behav. Ecol. Sociobiol. 50, 391402.

Borries, C., Larney, E., Kreetiyutanont, K., \& Koenig, A. (2002). The diurnal primate community in a dry evergreen forest in Phu Khieo Wildlife Sanctuary, Northeast Thailand. Nat. Hist. Bull.Siam Soc. 50, 75-88.

Brauch, K., Pfefferle, D., Hodges, K., Möhle, U., Fischer, J., \& Heistermann, M. (2007). Female sexual behavior and sexual swelling size as potential cues for males to discern the female fertile phase in free-ranging Barbary macaques (Macaca sylvanus) of Gibraltar. Horm. Behav. 52, 375-383.

Brauch, K., Hodges, J. K., Engelhardt, A., Fuhrmann, K., Shaw, E., \& Heistermann, M. (2008). Sex-specific reproductive behaviours and paternity in free-ranging Barbary macacaques (Macaca sylvanus). Behav. Ecol. Sociobiol. 62, 1453-1466.

Brockman, D. K., \& van Schaik, C. P. (2005). Seasonality and reproductive function. In: Brockman, D. K. \& van Schaik, C. P. (Eds.), Seasonality in Primates: Studies of Living and Extinct Human and Non-human Primates. Cambridge University Press, Cambridge. pp. 269-305.

Bruns, C. M., Baum, S. T., Colman, R. J., Eisner, J. R., Kemnitz, J. W., Weindruch, R., \& Abbott, D. H. (2004). Insulin resistance and impaired insulin secretion in prenatally androgenized male rhesus monkeys. J. Clin. Endocrinol. Metab. 89, 6218-6223. 
Buchan, J. C., Alberts, S. C., Silk, J. B., \& Altmann, J. (2003). True paternal care in a multimale primate society. Nature. 425, 179-181.

Burnham, K. P., \& Anderson, D. R. (2002). Model Selection and Multimodel Inference. 2nd ed. Berlin: Springer.

Burt, A. (1992). 'Concealed Ovulation' and Sexual Signals in Primates. Fol. Primatol. 58, 1-6.

Busse, C. D. (1985). Paternity recognition in multi-male primate groups. Am. Zool. 25, 873881.

Butler, H. (1974). Evolutionary trends in primate sex cycles. Contrib. Primatol. 3, 2-35.

Campbell, C. J., Fuentes, A., MacKinnon, K. C., Panger, M., \& Bearder, S. K. (2007). Primates in Perspective. Oxford University Press, Oxford, UK.

Carosi M., Heistermann M., \& Visalberghi E. (1999). Display of proceptive behaviors in relation to urinary and fecal progestin levels over the ovarian cycle in female tufted capuchin monkeys. Horm. Behav. 36, 252-265.

Castracane, V. D., \& Goldzieher, J. W. (1983). Plasma androgens during early pregnancy in the baboon (Papio cynocephalus). Fertil. Steril. 39, 553-559.

Castracane, V. D., \& Goldzieher, J. W. (1986). Timing of the luteal-placental shift in the baboon (Papio cynocephalus). Endocrinology. 118, 506-512.

Castracane, V. D., Stewart, D. R., Gimpel, T., Overstreet, J. W., \& Lasley, B. L. (1998). Maternal serum androgens in human pregnancy: early increases within the cycle of conception. Hum. Reprod. 13, 460-464.

Cerda-Molina, A., Hernández-López, L., Rojas-Maya, S., Murcia-Mejía, C., \& MondragónCeballos, R. (2006). Male-induced sociosexual behavior by vaginal secretions in Macaca arctoides. Int. J. Primatol. 26, 791-807.

Chambers, P. L., \& Hearn, J. P. (1979). Peripheral plasma concentrations of progesterone, oestradiol-17b, oestrone, testosterone, androstenedione and chorionic gonadotropin during pregnancy in the marmoset monkey Callithrix jacchus. J. Reprod. Fert. 56, 2332.

Clarke, P., Pradhan, G., \& van Schaik, C. P. (2009). Intersexual Conflict in Primates: Infanticide, Paternity Allocation, and the Role of Coercion. In: Muller, M. N. \& Wrangham, R. W. (Eds.), Sexual coercion in primates and humans: an evolutionary perspective on male agression against females. USA, pp. 42-80.

Clutton-Brock, T. H., \& Harvey, P. H. (1976). Evolutionary rules and primate societies. In: Bateson, P. P. G. \& Hinde, R. A. (Eds.), Growing Points in Ethology. Cambridge University Press, Cambridge. pp. 195-237.

Clutton-Brock, T. H., \& Vincent, A. C. J. (1991). Sexual selection and the potential reproductive rates of males and females. Nature 351, 58-60.

Cooper, M. A., \& Bernstein, I. S. (2002). Counter Aggression and Reconciliation in Assamese Macaques (Macaca assamensis). Am. J. Primatol. 56, 215-230.

Cords, M., Mitchell, B. J., Tsingalia, H. M., \& Rowell, T. E. (1986). Promiscuous mating among Blue monkeys in the Kakamega forest, Kenya. Ethology 72, 214-226. 
Cornell, S. J., \& Tregenza, T. (2007). A new theory for the evolution of polyandry as a means of inbreeding avoidance. Proc. R. Soc. B. 274, 2873-2879.

Coussi-Korbel, S., \& Fragaszy, D. (1995). On the relation between social dynamics and social learning. Anim. Behav. 50, 1441-1453

Cowlishaw, G., \& Dunbar, R. I. M. (1991). Dominance rank and mating success in male primates. Anim. Behav. 41, 1045-1056.

Darwin, C. (1871). The descent of man, and selection in relation to sex. John Murray, London.

Deschner, T., Heistermann, M., Hodges, J. K., \& Boesch, C. (2003). Timing and probability of ovulation in relation to sex skin swelling in wild West African chimpanzees, Pan troglodytes verus. Anim. Behav. 66, 551-560.

Deschner, T., Heistermann, M., Hodges, K., \& Boesch, C. (2004). Female sexual swelling size, timing of ovulation, and male behavior in wild West African chimpanzees. Horm. Behav. 46, 204-215

Dewsbury, D. A. (1982). Ejaculate cost and male choice. Am. Nat. 119, 601-610.

Dixon, A. F. (1983). Observations on the evolution and behavioural significance of "sexual skin" in female primates. Adv. Stud. Behav. 13, 63-106.

Dixson, A. F. (1998). Primate sexuality: Comparative studies of the prosimians, monkeys, apes, and human beings. Oxford: Oxford University Press.

Dobson, A. J. (2002). An Introduction to Generalized Linear Models. Chapman \& Hall/CRC, Boca Raton.

Domb, L. G., \& Pagel, M. (2001). Sexual swellings advertise female quality in wild baboons. Nature. 410, 204-206.

Doran-Sheehy, D. M., Fernadez, D., \& Borries, C. (2009). The strategic use of sex in wild female western gorillas. Am. J. Primatol. 71, 1011-1020.

Drea, C. M. (2011). Endocrine correlates of pregnancy in the ring-tailed lemur (Lemur catta): Implications for the masculinization of daughters. Horm. Behav. 59, 417-427.

Dubuc C., Brent, L. J. N., Accamando A. K., Gerald M. S., MacLarnon M., Semple S., Heistermann M., \& Engelhardt, A. (2009). Sexual skin color contains information about the timing of the fertile phase in free-ranging rhesus macaques. Int. J. Primatol. 30, 777-789.

Duer, C., Carden, M., Schmitt, D., \& Tomasi, T. (2002). Utility of maternal serum total testosterone analysis for fetal gender determination in Asian elephants (Elephas maximus). Anim. Reprod. Sci. 69, 47-52.

Eberhard, W. G. (1996). Female Control: Sexual Selection by Cryptic Female Choice. Princeton University Press, Princeton, NJ.

Ellinwood, W. E., Stanczyk, F. Z., Lazur, J. J., \& Novy, M. J. (1989). Dynamics of steroid biosynthesis during the luteal-placental shift in rhesus monkeys. J. Clin. Endocrinol. Metab. 69, 348-355. 
Emery, M. A., \& Whitten, P. L. (2003). Size of sexual swellings reflects ovarian function in chimpanzees (Pan troglodytes). Behav. Ecol. Sociobiol. 54, 340-351.

Emlen, S. T., \& Oring, L. W. (1977). Ecology, sexual selection, and the evolution of mating systems. Science. 197, 215-223.

Engelhardt, A., Pfeifer, J. B., Heistermann, M., Niemitz, C., van Hooff, J. A. R. A. M., \& Hodges, J. K. (2004). Assessment of female reproductive status by male longtailed macaques, Macaca fascicularis, under natural conditions. Anim. Behav. 67, 915-924.

Engelhardt, A., Hodges, J. K., Niemitz, C., \& Heistermann, M. (2005). Female sexual behavior, but not sex skin swelling is a reliable indicator for the timing of the fertile phase in wild long-tailed macaques (Macaca fascicularis). Horm. Behav. 47, 195-204.

Engelhardt, A., Hodges, J. K., \& Heistermann, M. (2007). Post-conception mating in wild long-tailed macaques (Macaca fascicularis): characterization, endocrine correlates and functional significance. Horm. Bahav. 51, 3-10.

French, J. A., \& Stribley, J. A. (1987). Synchronization of ovarian cycles within and between social groups in golden lion tamarins (Leontopithecus rosalia). Am. J. Primatol. 12, 469478.

French, J. A., Smith, A. S., \& Birnie, A. K. (2010). Maternal gestational androgen levels in female marmosets (Callithrix geoffroyi) vary across trimesters but do not vary with the sex ratio of litters. Gen. Comp. Endocrinol. 165, 309-314.

Frisch R. E., \& McArthur, J. W. (1974). Menstrual cycles: fatness as a determinant of minimum weight for height necessary for their maintenance or onset. Science. 185, 949-951.

Fujita, S., Mitsunaga, F., Sugiura, H., \& Shimizu, K. (2001). Measurement of urinary and fecal steroid metabolites during the ovarian cycle in captive and wild Japanese macaques, Macaca fuscata. Am. J. Primatol. 53, 167-176.

Fürtbauer, I., Schülke, O., Heistermann, M., \& Ostner, J. (2010). Reproductive and life history parameters of wild female Macaca assamensis. Int. J. Primatol. 31, 501-517.

Fürtbauer, I., Heistermann, M., Schülke, O., \& Ostner, J. (submitted). Concealed fertility and extended female sexuality in a non-human primate (Macaca assamensis). PLoS ONE

Gangestad, S. W., \& Thornhill, R. (2008). Human oestrus. Proc. R. Soc. B. 275, 991-1000.

Gattermann, R., Ulbrich, K., \& Weinandy, R. (2002). Asynchrony in the estrous cycles of golden hamsters (Mesocricetus auratus). Horm. Behav. 42, 70-77.

Gibori, G., \& Sridaran, R. (1981). Sites of androgen and estradiol production in the second half of pregnancy in the rat. Biol. Reprod. 24, 249-256.

Girard-Buttoz, C., Heistermann, M., Krummel, S., \& Engelhardt, A. (2009). Seasonal and social influences on fecal androgen and glucocorticoid excretion in wild male long-tailed macaques (Macaca fascicularis). Physiol. Behav. 98, 168-75.

Glass, A. R., \& Klein, T. (1981). Changes in maternal serum total and free androgen levels in early pregnancy: lack of correlation with fetal sex. Am. J. Obstet. Gynecol. 140, 656660. 
Gowaty, P. A. (2004). Sex roles, contests for the control of reproduction and sexual selection. In: Kappeler, P. (Ed.), Sexual Selection in Primates, Cambridge University Press, Cambridge, pp. 37-54.

Goy, R. W., Bercovitch, F. B., \& McBrair, M. C. (1988). Behavioral masculinization is independent of genital masculinization in prenatally androgenized female rhesus macaques. Horm. Behav. 22, 552-571.

Grassman, L. I., Jr., Tewes, M. E., Silvy, N. J, \& Kreetiyutanont, K. (2005). Spatial organization and diet of the leopard cat (Prionailurus bengalensis) in north-central Thailand. J. Zool. (London). 266, 45-54.

Gray, J. P., \& Wolfe, L. D. (1983). Human female sexual cycles and the concealment of ovulation problem. J. Social. Biol. Struct. 6, 345-352.

Hamilton, W.J. (1984). Significance of paternal investment by primates to the evolution of male-female associations. In: Taub, D.M. (Ed.), Primate paternalism. New York; Van Nostrand Rheinhold, pp. 57-74.

Handelmann, G., Ravizza, R., \& Ray, W. J. (1980). Social dominance determines estrous entrainment among female hamsters. Horm. Behav. 14, 107-115.

Heape, W. (1900). "The 'sexual season' of mammals and the relation of the 'pro-oestrum' to menstruation'". Quart. Journ. Micr. Sci. 44, 1-70.

Heistermann, M., \& Hodges, J. K. (1995). Endocrine monitoring of the ovarian cycle and pregnancy in the saddle-back tamarin (Saguinus fuscicollis) by measurement of steroid conjugates in urine. Am. J. Primatol. 35, 117-127.

Heistermann, M., Tari, S., \& Hodges, J. K. (1993). Measurement of faecal steroids monitoring ovarian function in New World primates, Callitrichidae. J. Reprod. Fert. 99, 243-251.

Heistermann, M., Finke, M., \& Hodges, J. K. (1995). Assessment of female reproductive status in captive-housed Hanuman langurs (Presbytis entellus) by measurement of urinary and fecal steroid excretion patterns. Am. J. Primatol. 37, 275-284.

Heistermann, M., Ziegler, T., van Schaik, C. P., Launhardt, K., Winkler, P., \& Hodges, J. K. (2001a). Loss of oestrus, concealed timing of ovulation and paternity confusion in freeranging Hanuman langurs, Proc. $R$. Soc. B. 268, 2445-2451.

Heistermann, M., Uhrigshardt, J., Husung, A., Kaumanns, A., \& Hodges, J. K. (2001b). Measurement of faecal steroid metabolites in the lion-tailed macaque (Macaca silenus): A non-invasive tool for assessing female ovarian function. Primate Report. 59, 27-42.

Heistermann, M., Brauch, K., Möhle, U., Pfefferle, D., \& Hodges, K. (2008). Female ovarian cycle phase affects the timing of male sexual activity in free-ranging Barbary macaques (Macaca sylvanus) of Gibraltar. Am. J. Primatol. 70, 44-53.

Higham, J. P., MacLarnon, A. M., Ross, C., Heistermann, M., \& Semple, S. (2008). Baboon sexual swellings: Information content of size and color. Horm. Behav. 53, 452-462.

Higham, J. P., Semple, S., MacLarnon, A., Heistermann, M., \& Ross, C. (2009). Female reproductive signaling, and male mating behavior, in the olive baboon. Horm. Behav. $55,60-67$. 
Hill, D. A. (1997). Seasonal Variation in the Feeding Behavior and Diet of Japanese Macaques (Macaca fuscata yakui) in Lowland Forest of Yakushima. Am. J. Primatol. 43, 305-323.

Hodgen, G. D., \& Itskovitz, J. (1988). Recognition and maintenance of pregnancy. In: Knobil, E. \& Neill, J. (Eds.), The Physiology of Reproduction, Raven Press, New York, pp. 1995-2021.

Hodges, J. K., Tarara, R., \& Wangula, C. (1984). Circulating steroids and the relationship between ovarian and placental secretion during early and mid pregnancy in the baboon. Am. J. Primatol. 7, 357-366.

Hoelzer G. A., \& Melnick D. J. (1996). Evolutionary relationships of the macaques. In: Fa, J. E. \& Lindburg, D. G. (Eds.), Evolution and ecology of macaque societies. New York: Cambridge Univ Press. pp.3-19.

Hrdy, S. B. (1979). Infanticide among animals: a review, classification, and examination of the implications for the reproductive strategies of females. Ethol. Sociobiol. 1, 13-40.

Hrdy, S. B. (2000). The optimal number of fathers: evolution, demography, and history in the shaping of female mate preferences. Ann. New York Acad. Sci. 907, 75-96.

Hrdy, S. B., \& Hausfater, G. (1984). Comparative and evolutionary perspectives on infanticide: introduction and overview. In: Infanticide: Comparative and Evolutionary Perspectives. Hrdy, S. \& Hausfater, G. (Eds.) New York, Aldine Publishing Company, pp. 13-35.

Hrdy, S. B., \& Whitten, P. L. (1987). Patterning of sexual activity. In: Smuts, B. B., Cheney, D. L., Seyfarth, R. M., Wrangham, R. W. \& Struhsaker, T. T. (Eds.), Primate Societies. Chicago, University of Chicago Press. pp. 370-384.

Ims, R. A. (1988). The potential for sexual selection in males: effect of sex ratio and spatiotemporal distribution of receptive females. Evol. Ecol. 2, 338-352.

Ims, R. A. (1990). The ecology and evolution of reproductive synchrony. Trends Ecol. Evol. $5,135-140$.

Jennions, M. D., \& Petrie, M. (2000). Why do females mate multiply? A review of the genetic benefits. Biol. Rev. Cambridge. Philosoph. Soc. 75, 21-64.

Jin, T., Wang, D. Z., Zhao, Q., Yin, L. J., Qin, D. G., Ran, W. Z., \& Pan, W. S. (2009). Reproductive parameters of wild Trachypithecus leucocephalus: seasonality, infant mortality and interbirth interval. Am. J. Primatol. 71, 558-566.

Kappeler, P. M., \& Pereira, M. E. (2003). Primate life histories and socioecology. Chicago: University of Chicago Press.

Kappeler, P. M., Pereira, M. E., \& van Schaik, C. P. (2003). Primate life histories and socioecology. In: Kappeler, P. M. \& Pereira, M. E. (Eds.), Primate life histories and socioecology. Chicago: University of Chicago Press. pp. 1-20.

Knobil E, Neill JD. 2006. Physiology of reproduction. Boston: Elsevier Inc. 3230 pp.

Knowlton, N. (1979). Reproductive synchrony, parental investment, and the evolutionary dynamics of sexual selection. Anim. Behav. 27, 1022-1033. 
Koenig, A., Borries, C., Suarez, S., Kreetiyutanont K., \& Prabnasuk, J. (2004). Socio-ecology of Phayre's leaf monkeys (Trachypithecus phayrei) at Phu Khieo Wildlife Sanctuary. Journal of Wildlife in Thailand. 12, 150-163.

Küster, J., \& Paul, A. (1984). Female reproductive characteristics in semifree-ranging Barbary macaques (Macaca sylvanus L. 1758). Fol. Primatol. 43, 69-83.

Kutsukake, N., \& Nunn, C. L. (2006). Comparative tests of reproductive skew in male primates: the roles of demographic factors and incomplete control. Behav. Ecol. Sociobiol. 60, 695-706.

Lee, P. C. (1996). The meanings of weaning: Growth, lactation and life history. Evol. Anthropol. 5, 87-96.

Lee, P. C. (1999). Comparative ecology of postnatal growth and weaning among haplorhine primates. In: Lee, P. C. (Ed.), Comparative Primate Socioecology. Cambridge: Cambridge Univ Press. pp. 111-139.

Lee P. C., \& Kappeler, P. M. (2003). Socioecological correlates of phenotypic plasticity of primate life histories. In: Kappeler, P. M. \& Pereira, M. E. (Eds.), Primate life histories and socioecology. Chicago: University of Chicago Press. pp. 41-65.

Lee, P. C., Majluf, P., \& Gordon, I. J. (1991). Growth, weaning and maternal investment from a comparative perspective. J. Zool. 225, 99-114.

Legrand, C., Marie, J., \& Maltier, J. P. (1984). Testosterone, dihydrotestosterone, androstenedione and dehydroepiandrosterone concentrations in placentae, ovaries and plasma of the rat in late pregnancy. Acta. Endocrinol. 105, 119-125.

Lewis, R. J., \& Kappeler, P. M. (2005). Seasonality, body condition, and timing of reproduction in Propithecus verreauxi verreauxi in the Kirindy Forest. Am. J. Primatol. 67, 347-364.

Li, J. H., Yin, H. B., \& Wang, Q. S. (2005). Seasonality of reproduction and sexual activity in female Tibetan macaques Macaca thibetana at Huangshan, China. Acta Zool. Sin. 51, 365-375.

Lindburg, D. G. (1971). The rhesus monkey in north India; an ecological and behavioral study. In: Rosenblum, L. A. (Ed.), Primate Behavior: Development in Field and Laboratory Research. Academic Press, New York, 2, pp. 1-106.

Lockhart, A. B., Thrall, P.H., \& Antonovics, J. (1996). Sexually transmitted diseases in animals: ecological and evolutionary implications. Biol. Rev. Camb. Phil. Soc. 71, 415471.

Maestripieri, D., \& Roney, J. R. (2005). Primate copulation calls and postcopulatory female choice. Behav. Ecol. 16, 106-113.

Maninger, N., Sackett, G. P., \& Ruppenthal, G. C. (2000). Weaning, body weight, and postpartum amenorrhea duration in pigtailed macaques (Macaca nemestrina). Am. J. Primatol. 52, 81-91.

Manson, J. H. (1992). Measuring female mate choice in Cayo Santiago rhesus macaques. Anim. Behav. 44, 405-416.

Manson, J. H. (1997). Primate Consortships: A Critical Review. Curr. Anthropol. 38, 353-374. 
Marson, J., Gervais, D., Cooper, R. W., \& Jouannet, P. (1989). Influence of ejaculation frequency on semen characteristics in chimpanzees (Pan troglodytes). J. Reprod. Fertil. 85, 43-50.

Matsubara, M., \& Sprague, D. S. (2004). Mating tactics in response to costs incurred by mating with multiple males in wild female Japanese macaques. Int. J. Primatol. 25, 901-917.

Matsumoto-Oda, A., \& Kasuya, E. (2005). Proximity and Estrous Synchrony in Mahale Chimpanzees. Am. J. Primatol. 66, 159-166.

Matsumoto-Oda, A., Hamai, M., Hayaki H., Hosaka K., Hunt K. D., Kasuya E., Kawanaka K., Mitani J. C., Takasaki, H., \& Takahata, Y. (2007). Estrus cycle asynchrony in wild female chimpanzees, Pan troglodytes schweinfurthii. Behav. Ecol. Sociobiol. 61, 661668.

McCarthy, M., \& Becker, J. B. (2002). Neuroendocrinology of sexual behavior in the female. In: Becker, J., Breedlove, S., Crews, D., \& McCarthy, M. (Eds.), Behavioral Endocrinology. The MIT Press, Cambridge, pp. 117-151.

McClintock, M. K. (1971). Menstrual synchrony and suppression. Nature. 229, 244-245.

McClintock, M. K. (1978). Estrous synchrony and its mediation by airborne chemical communication (Rattus norvegicus). Horm. Behav. 10, 264-276.

McClintock, M. K. (1984). Estrous synchrony: modulation of ovarian cycle length by females pheremone. Physiol. Behav. 32, 701-705.

McNeilly, A. S. (2001). Lactational control of reproduction. Reprod. Fertil. Dev. 13, 583-590.

Melnick, D. J., \& Pearl, M. C. (1987). Cercopithecines in multimale groups: Genetic diversity and population structure. In: Smuts, B. B., Cheney, D. L., Seyfarth, R. M., Wrangham R. W. \& Struhsaker, T. T. (Eds.), Primate Societies. Chicago, IL: University of Chicago Press. pp. 121-134.

Ménard, N., \& Vallet, D. (1996). Demography and ecology of Barbary macaques (Macaca sylvanus) in two different habitats. In Fa, J. E. \& Lindburg, D. G. (Eds.), Evolution and ecology of macaque societies. New York: Cambridge Univ Press. pp.106-131.

Meulenberg, P. M. M., \& Hofman, J. A. (1991). Maternal testosterone and fetal sex. J. Ster. Biochem. Mol. Biol. 39, 51-54.

Monfort, S. L., Bush, M., \& Wildt, D. E. (1996). Natural and induced ovarian synchrony in golden lion tamarins (Leontopithecus rosalia). Biol. Reprod. 55, 875-882.

Moscovice, L. R., Di Fiore, A., Crockford, C., Kitchen, D. M., Wittig, R., Seyfarth, R. M., \& Cheney, D. L. (2010). Hedging their bets? Male and female chacma baboons form friendships based on likelihood of paternity. Anim. Behav. 79, 1007-1015.

Möhle, U., Heistermann, M., Palme, R., \& Hodges, J. K. (2002). Characterization of urinary and fecal metabolites of testosterone and their measurement for assessing gonadal endocrine function in male nonhuman primates. Gen. Comp. Endocrinol. 129, 135-145. 
Möhle, U., Heistermann. M., Dittami, J., Reinberg, V., Wallner, B., \& Hodges, J. K. (2005). Patterns of anogenital swelling size and their endocrine correlates during ovulatory cycles and early pregnancy in free-ranging Barbary macaques (Macaca sylvanus) of Gibraltar. Am. J. Primatol. 66, 351-68.

Muller, M. N., \& Wrangham, R. W. (2009) Sexual coercion in primates and humans. An Evolutionary Perspective on Male Aggression Against Females. Harvard University Press, Cambridge, MA.

Muller, M. N., Emery Thompson, M., Kahlenberg, S. M., Wrangham, R. W. (2011). Sexual coercion by male chimpanzees shows that female choice may be more apparent than real. Behav. Ecol. Sociobiol. 65, 921-933.

Nelson, R. J. (2005). An Introduction to Behavioral Endocrinology. Third ed. Sunderland, MA, USA: Sinauer Associates.

Nonacs, P. (2000). Measuring and using skew in the study of social behavior and evolution. Am. Nat. 156, 577-589.

Nunn, C. L. (1999a). The number of males in primate social groups: a comparative test of the socioecological model. Behav. Ecol. Sociobiol. 46, 1-13.

Nunn, C. L. (1999b). The evolution of exaggerated swellings in primates and the gradedsignal hypothesis. Anim. Behav. 58, 229-246.

Nunn, C. L, Gittleman, J. L., \& Antonovics, J. (2000). Promiscuity and the primate immune system. Science. 290, 1168-1170.

Nunn, C. L., van Schaik, C. P., \& Zinner, D. (2001). Do exaggerated sexual swellings function in female mating competition in primates? A comparative test of the reliable indicator hypothesis. Behav. Ecol. 12, 646-654.

Okamoto, K. (2000). Life history and demography of wild moor macaques (Macaca maurus): Summary of ten years of observations. Am. J. Primatol. 52, 1-11.

O'Neill, A. C., Fedigan, L. M., \& Ziegler, T. E. (2004). Relationship between ovarian cycle phase and sexual behavior in female Japanese macaques (Macaca fuscata). Am. J. Phys. Anthropol. 125, 352-362.

Ostner, J., Heistermann, M., \& Kappeler, P. M. (2003). Intersexual dominance, masculinized genitals and prenatal steroids: comparative data from lemurid primates. Naturwissenschaften. 90, 141-144.

Ostner, J., Chalise, M. K., Koenig, A., Launhardt, K., Nikolei, J., Podzuweit, D., \& Borries, C. (2006). What Hanuman langur males know about female reproductive status. Am. J. Primatol. 68, 701-712.

Ostner, J., Nunn, C. L., \& Schülke, O. (2008a). Female reproductive synchrony predicts skewed paternity across primates. Behav. Ecol. 19, 1150-1158.

Ostner, J., Heistermann, M., \& Schülke, O. (2008b). Dominance, aggression and physiological stress in wild male Assamese macaques (Macaca assamensis). Horm. Behav. 54, 613-619.

Ostner, J., Heistermann, M., \& Schülke, O. (2011). Male competition and its hormonal correlates in Assamese macaques (Macaca assamensis). Horm. Behav. 59, 105-113. 
Pagel, M. (1994). The evolution of conspicuous oestrus advertisement in Old World monkeys. Anim. Behav. 47,1333-1341.

Palme, R., \& Möstl, E. (1994). Biotin-straptavidin enzyme immunoassay for the determination of oestrogens and androgens in boar faeces. In: Görög, S. (Ed.), Advances in Steroid Analysis. Akadémiai Kiadó, Budapest, pp. 11-117.

Palombit, R. A., Cheney, D. L., Fischer, J., Johnson, S., Rendall, D., Seyfarth, R. M. \& Silk, J. B. (2000). Male infanticide and the defense of infants in chacma baboons. In: van Schaik, C. P. \& Janson, C. H. (Eds.), Infanticide by Males and its Implications, Cambridge: Cambridge University Press. pp. 123-152.

Panova, M., Boström, J., Hofving, T., Areskoug, T., Eriksson, A., Mehlig B., Mäkinen, T., André, C., \& Johannesson, K. (2010). Extreme Female Promiscuity in a Non-Social Invertebrate Species. PLoS ONE 5(3): e9640. doi: 10.1371/journal.pone.0009640.

Parker, G. A. (1979). Sexual selection and sexual conflict. In: Blum, M. S. \& Blum, N. A. (Eds.), Sexual Selection and Reproductive Competition in Insects, New York: Academic Press. pp. 123-166.

Paul, A. (1997) Breeding seasonality affects the association between dominance and reproductive success in non-human male primates. Fol. Primatol. 68, 344-349.

Paul, A., Preuschoft, S., \& van Schaik, C. P. (2000). The other side of the coin: infanticide and the evolution of affiliative male-infant interactions in Old World primates. In: van Schaik, C. P. \& Janson, C. (Eds.), Infanticide by males and its implications. Cambridge University Press, Cambridge, pp. 269-292.

Pereira, M. E. (1991). Asynchrony within estrous synchrony among ringtailed lemurs (Primates: Lemuridae). Physiol. Behav. 49, 47-52.

Phoenix, C. H., Goy, R. W., Gerall, A. A., \& Young, W. C. (1959). Organizing action of prenatally administered testosterone propionate on the tissues mediating mating behavior in the female guinea pig. Endocrinology. 65, 369-382.

Pradhan, G. R., \& van Schaik, C. P. (2008). Infanticide-driven intersexual conflict over matings in primates and its effects on social organization. Behaviour. 145, 251-275.

Preston, B. T., Stevenson, I. R., Pemberton, J. M., \& Wilson, K. (2001). Dominant rams lose out by sperm depletion. Nature. 409, 681-682.

R Development Core Team (2008). R: A language and environment for statistical computing. R Foundation for Statistical Computing, Vienna, Austria.

R Development Core Team (2010). R: A language and environment for statistical computing. R Foundation for Statistical Computing, Vienna, Austria.

Radespiel, U., \& Zimmermann, E. (2001). Dynamics of Estrous Synchrony in Captive Gray Mouse Lemurs (Microcebus murinus). Int. J. Primatol. 22, 71-90.

Rao, A. J., \& Kotagi, S. G. (1983). Serum testosterone levels during the menstrual cycle and early pregnancy in the bonnet monkey (Macaca radiata). Endocrinol. Jpn. 29, 271-275.

Resko, J. A., \& Ellinwood, W. E. (1981). Testicular hormone production in fetal rhesus macaques. In: Novy, M. J. \& Resko, J. A. (Eds.), Fetal Endocrinology. Academic Press, New York, pp. 253-269. 
Reynolds, J. D., \& Gross, M. R. (1990). Costs and benefits of female mate choice: Is there a lek paradox? Am. Nat. 136, 230-243.

Ridley, M. (1986). The number of males in a primate troop. Anim. Behav. 34, 1848-1858.

Robbins, M. M., Robbins, A. M., Gerald-Steklis, N., \& Steklis, H. D. (2007). Socioecological influences on the reproductive success of female mountain gorillas (Gorilla beringei beringei). Behav. Ecol. Sociobiol. 61, 919-931.

Roberts, E. K., Flak, J. N., Ye, W., Padmanabhan, V., \& Lee, T. M. (2009). Juvenile rank can predict male-typical adult mating behavior in female sheep treated prenatally with testosterone. Biol. Reprod. 80, 737-742.

Rodriguez-Gironés, M. A., \& Enquist, M. (2001). The evolution of female sexuality. Anim. Behav. 61, 695-704.

Rowell, T. E., \& Hartwell, K. M. (1978). The interaction of behaviour and reproductive cycles in patas monkeys. Behav. Biol. 24, 141-167.

Russell, M. J., Switz, G. M., \& Thompson, K. (1980). Olfactory influences on the human menstrual cycle. Pharmacol. Biochem. Behav. 13, 737-738.

Schank, J. C. (2001a). Do Norway rats (Rattus norvegicus) synchronize their estrous cycles? Physiol. Behav. 72, 12-139.

Schank, J. C. (2001b). Measurement and cycle variability: reexamining the case for ovarian cycle synchrony in primates. Behav. Proc. 56, 131-146.

Schank, J. C. (2001c). Menstrual-cycle synchrony: problems and new directions for research. J. Comp. Psychol. 115, 3-15.

Schülke, O., Bhagavatula, J., Vigilant, L., \& Ostner, J. (2010). Social bonds enhance reproductive success in male macaques. Curr. Biol. 20, 2207-2210.

Schwensow, N., Eberle, M. \& Sommer, S. (2008). Compatibility counts: MHC-associated mate choice in a wild promiscuous primate. Proc. $R$. Soc. B. $275,555-564$.

Semple, S., \& McComb, K. (2000). Perception of female reproductive state from vocal cues in a mammal species. Proc. $R$. Soc. B. 267, 707-712.

Semple, S., McComb, K., Alberts, S., \& Altmann, J. (2002). Information Content of Female Copulation Calls in Yellow Baboons. Am. J. Primatol. 56, 43-56.

Setchell, J. M., Kappeler, P. M. (2003). Selection in relation to sex in primates. Adv. Stud. Behav. 33, 87-173.

Setchell, J. M., Kendal, J., \& Tyniec, P. (2010a). Do non-human primates synchronise their menstrual cycles? A test in mandrills. Psychoneuroendocrinology. 36, 51-59.

Setchell, J. M., Charpentier, M. J. E., Abbott, K. M., Wickings, E. J., \& Knapp, L. A. (2010b). Opposites attract: MHC-associated mate choice in a polygynous primate. J. Evol. Biol. 23, 136-148.

Setchell, J. M., Lee. P. C., Wickings, E. J., \& Dixson, A. F. (2002). Reproductive parameters and maternal investment in mandrills (Mandrillus sphinx). Int. J. Primatol. 23, 51-68. 
Shideler, S. E., Orturo, A. M., Moran, F. M., Moorman, E. A., \& Lasley, B. L. (1993). Simple extraction and enzyme immunoassays for estrogen and progesterone metabolites in the feces of Macaca fascicularis during non-conceptive and conceptive ovarian cycles. Biol. Reprod. 48, 1290-1298.

Silk, J. B. (1990). Sources of variation in interbirth intervals among captive Bonnet Macaques (Macaca radiata). Am. J. Phys. Anthropol. 82, 213-230.

Small, M. (1988). Female primate sexual behaviour and conception. are there really sperm to spare? Curr. Anthropol. 29, 81-100.

Small, M. F. (1990). Promiscuity in Barbary Macaques (Macaca sylvanus). Am. J. Primatol. 20, 267-282.

Smith, A. S., Birnie, A. K., \& French, J. A. (2010). Maternal androgen levels during pregnancy are associated with early-life growth in Geoffroy's marmosets, Callithrix geoffroyi. Gen. Comp. Endocrinol. 166, 307-313.

Smuts, B. B. (1985). Sex and Friendship in Baboons. New York: Aldine.

Smuts, B. B., \& Smuts, R. W. (1993). Male aggression and sexual coercion of females in nonhuman primates and other mammals: evidence and theoretical implications. Adv. Stud. Behav. 22, 1-63.

Sokal, R. R., \& Rohlf, F. J. (1995). Biometry - The Principles and Practice of Statistics in Biological Research, 3rd edn. Freeman \& Co. New York.

Soltis, J. (2002). Do primate females gain nonprocreative benefits by mating with multiple males? Theoretical and empirical considerations. Evol. Anthropol. 11, 187-197.

Soltis, J. \& McElreath, R. (2001). Can Females Gain Extra Paternal Investment by Mating with Multiple Males? A Game Theoretic Approach. Amer. Nat. 158, 1519-529.

Soltis, J., Mitsunaga, F., Shimizu, K., Yanagihara, Y., \& Nozaki, M. (1999). Female mating strategy in an enclosed group of Japanese macaques. Am. J. Primatol. 47, 263-278.

Soltis, J., Thomsen, R., Matsubayashi, K., \& Takenaka, O. (2000). Male infanticide by resident males and female counter-strategies in wild Japanese macaques (Macaca fuscata). Behav. Ecol. Sociobiol. 48, 195-202.

Stacey, P. B. (1982). Female promiscuity and male reproductive success in social birds and mammals. Amer. Nat. 120, 51-64.

Steler, J. A., Ulstein, M., \& Myking, O. L. (2002). Human chorionic gonadotropin and testosterone in normal and preeclamptic pregnancies in relation to fetal sex. Obstet. Gynecol. 100, 552-556.

Stern, K., \& McClintock, M. K. (1998). Regulation of ovulation by human pheremones. Nature. 392, 177-189.

Stoinski, T. S., Perdue, B. M., \& Legg, A .M. (2009). Sexual behaviour in female western lowland gorillas (Gorilla gorilla gorilla): evidence for sexual competition. Am. J. Primatol. 71, 587-593. 
Strassmann, B. I. (1997). The biology of menstruation in Homo sapiens: total lifetime menses, fecundity, and nonsynchrony in a natural fertility population. Curr. Anthropol. $38,123-129$.

Strassman, B. I. (1981). Sexual selection, paternal care, and concealed ovulation in humans. Ethol. Sociobiol. 2, 31-40.

Strier, K. B., \& Ziegler, T. E. (1997). Behavioral and endocrine characteristics of the reproductive cycle in wild muriqui Monkeys, Brachyteles arachnoides. Am. J. Primatol. 42, 299-310.

Stumpf, R. M., Martinez-Mota, R., Milich, K. M., Righini, N., \& Shattuck, M. R. (2011). Sexual conflict in primates. Evol. Anthropol. 20, 62-75.

Takahata, Y. (1980). The reproductive biology of a free-ranging troop of Japanese monkeys. Primates. 21, 303-329.

Takahata, Y., Suzuki, S., Agetsuma N., Okayasu, N., Sugiura, H., Takahashi, H., Yamagiwa, J., Izawa, K., Furuichi, T., Hill, D. A., Maruhashi, T., Saito, C., Sato, S., \& Sprague, D. S. (1998). Reproduction of Wild Japanese Macaque Females of Yakushima and Kinkazan Islands: A Preliminary Report. Primates. 39, 339-349.

Tanaka T., Tokuda, K., \& Kotera, S. (1970). Effects of infant loss on the interbirth interval of Japanese monkeys. Primates. 11, 113-117.

Taub, D. M. (1980). Female choice and mating strategies among wild Barbary macaques (Macaca sylvanus). In: Lindburgh, D. G. (Ed.), The Macaques: Studies in Ecology, Behavior and Evolution, Van Nostrand Reinhold Company, New York, pp. 287-344.

Thierry, B. (2007). The Macaques: a Double-layered Social Organization. In: Campbell, C., Fuentes, A. \& MacKinnon, K. (Eds.), Primates in Perspective. Oxford University Press, Oxford. pp. 224-239.

Thierry, B., Iwaniuk, A. N., \& Pellis, S. M. (2000). The influence of phylogeny on the social behaviour of macaques (Primates: Cercopithecidae, genus Macaca). Ethology. 106, 713-728.

Thomsen, R., Soltis, J., Matsubara, M., Matsubayashi, K., Onuma, M., \& Takenaka, O. (2006). How costly are ejaculates for Japanese macaques? Primates. 47, 272-274.

Thornhill, R., \& Gangestad, S. W. (2008). The Evolutionary Biology of Human Female Sexuality. Oxford University Press, New York, NY.

Thornton J., Zehr, J. L., \& Loose, M. D. (2009). Effects of prenatal androgens on rhesus monkeys: a model system to explore the organizational hypothesis in primates. Horm. Behav. 55, 633-644.

Tobler, R., Pledger, S., \& Linklater, W. (2010). No evidence for ovarian synchrony or asynchrony in hamadryas baboons. Anim. Behav. 80, 829-837.

Tomaszycki, M. L., Davis, J. E., Gouzoules, H., \& Wallen, K. (2001). Sex differences in infant rhesus macaque separation-rejection vocalizations and effects of prenatal androgens. Horm. Behav. 39, 267-76.

Trivers, R. L. (1972). Parental investment and sexual selection. In: Campbell, B. (Ed.), Sexual selection and the descent of man, 1871-1971. Chicago, Aldine. pp. 136-179. 
Troisi, R., Potischman, N., Roberts, J. M., Harger, G., Markovic, N., Cole, B., Lykins, D., Siiteri, P., \& Hoover, R. N. (2003). Correlation of serum hormone concentrations in maternal and umbilical cord samples. Cancer Epidimiol. Biomarkers Prev. 12, 452-456.

Uller, T., \& Olsson, M. (2008). Multiple paternity in reptiles: patterns and processes. Mol. Ecol. 17, 2566-2580.

Vandenbergh, J. G., \& Drickamer, L. C. (1974). Reproductive coordination among freeranging rhesus monkeys. Physiol. Behav. 13, 373-376.

van Hooff, J. (1967). The facial displays of the catarrhine monkeys and apes. In D. Morris (Ed.), Primate Ethology. pp. 7-68.

van Nordwijk, M. (1985). Sexual Behaviour of Sumatran Long-tailed Macaques (Macaca fascicularis). Zeitschrift für Tierpsychologie. 70, 277-296.

van Nordwijk, M. A., \& van Schaik, C. P. (2004). Sexual selection and the careers of primate males: paternity concentration, dominance-acquisition tactics and transfer decisions. In: Kappeler, P. M. \& van Schaik, C. P. (Eds.), Sexual Selection in Primates: New and Comparative Perspectives, Cambridge University Press Cambridge. pp. 208-229.

van Noordwijk, M. A., \& van Schaik, C. P. (2000). Reproductive patterns in eutherian mammals: Adaptations against infanticide? In: van Schaik, C. P. \& Janson, C. H. (Eds.), Infanticide by Males and its Implications, Cambridge University Press, Cambridge, pp. 322-360.

van Schaik, C. P. (2000a). Vulnerability to infanticide by males: Patterns among mammals. In: van Schaik, C.P. \& Janson, C.H. (Eds.), Infanticide by Males and its Implications, Cambridge University Press, Cambridge, pp. 61-71.

van Schaik, C. P. (2000b). Social counterstrategies against infanticide by males in primates and other mammals. In: Kappeler, P. M. (Ed.), Primate Males, Cambridge University Press, Cambridge, pp. 34-52.

van Schaik, C. P, Janson, C. H. (2000). Infanticide by Males and its Implications, Cambridge University Press, Cambridge

van Schaik, C. P., van Noordwijk, M. A, \& Nunn C. L. (1999). Sex and social evolution in primates. In: Lee, P.C. (Ed.), Comparative Primate Socioecology Cambridge University Press, Cambridge, pp. 204-231.

van Schaik, C. P, Hodges K. J., \& Nunn, C. (2000). Paternity confusion and the ovarian cycles of female primates. In: van Schaik, C. P. \& Janson, C. H. (Eds.), Infanticide by males and its implications. Cambridge University Press, Cambridge, pp. 361-387.

van Schaik, C. P., Pradhan, G. R., \& van Noordwijk, M. A. (2004). Mating conflict in primates: infanticide, sexual harassment and female sexuality. In: Kappeler, P. M. \& van Schaik, C. P. (Eds.), Sexual Selection in Primates: New and Comparative Perspectives, Cambridge University Press, Cambridge, pp. 131-150.

von Engelhardt, N., Kappeler; P. M., \& Heistermann, M. (2000). Androgen levels and female social dominance in Lemur catta. Proc. R. Soc. B. 267, 1533-1539.

Walker, M. L., Gordon, T. P., \& Wilson, M. E. (1983). Menstrual cycle characteristics of seasonally breeding rhesus monkeys. Biol. Reprod. 29, 841-848. 
Wallen, K. (1990). Desire and ability: Hormones and the regulation of female sexual behavior. Neurosci. Biobehav. Rev. 14, 233-241.

Wallen, K. (1996). Nature needs nurture: the interaction of hormonal and social influences on the development of behavioral sex differences in rhesus monkeys. Horm. Behav. 30, 364-378.

Wallen, K. (2005). Hormonal influences on sexually differentiated behavior in nonhuman primates. Front. Neuroendocrinol. 26, 7-26.

Wallen, K., \& Hassett, J. M. (2009). Sexual differentiation of behavior in monkeys: role of prenatal hormones. J. Neuroendocrinol. 21, 421-426.

Wallis, J. (1985). Synchrony of estrous swelling in captive group-living chimpanzees (Pan troglodytes). Int. J. Primatol. 6, 335-350.

Wasser, S., Hunt, K., Brown, J., Cooper, K., Crockett, C., Bechert, U., Millspaugh, J., Larson, S., \& Monfort, S. (2000). A generalized fecal glucocorticoid assay for use in a diverse array of nondomestic mammalian and avian species. Gen. Comp. Endocrinol. 120, 260-275.

Wehrenberg, W. B., Dyrenfurth, I., \& Ferin, M. (1980). Endocrine characteristics of the menstrual cycle in the Assamese Monkey (Macaca assamensis). Biol. Reprod. 23, 522525.

Weingrill, T., Lycett, J. E., Hill, R. A., Barrett, L., \& Henzi, S. P. (2003). Male consortship behaviour in chacma baboons: the role of demographic factors and female conceptive probabilities. Behaviour. 140, 405-427.

Whitten, P. L. (1987). Infants and adult males. In: Smuts, B. B., Cheney, D. L., Seyfarth, R. M., Wrangham, R. W. \& Struhsaker, T. T. (Eds.), Primate Societies. Chicago, University of Chicago Press. pp. 343-357.

Whitten, P. L., Brockman, D. K., \& Stavisky, R. C. (1998). Recent advances in noninvasive techniques to monitor hormone-behavior interactions. Am. J. Phys. Anthropol. 27, 123.

Wolf, C. J., Hotchkiss, A., Ostby, J. S., LeBlanc, G. A., \& Gray, L. E. (2002). Effects of prenatal testosterone propionate on the sexual development of male and female rats: a dose-response study. Toxicol. Sci. 65, 71-86.

Wolff, J. O., \& Macdonald, D. W. (2004). Promiscuous females protect their offspring. Trends Ecol. Evol. 19, 127-133.

Yasui, Y. (1997). A "good-sperm" model can explain the evolution of costly multiple mating by females. Amer. Nat. 149, 573-584.

Zhao, Q. K., \& Deng, Z. Y. (1988). Macaca thibetana at Mt. Emei, China: II. Birth seasonality. Am. J. Primatol. 16, 261-268.

Ziegler, T., Hodges, K., Winkler, P., \& Heistermann, M. (2000). Hormonal correlates of reproductive seasonality in wild Hanuman langurs (Presbytis entellus). Am. J. Primatol. 51, 119-134. 
Zinner, D., \& Deschner, T. (2000). Sexual Swellings in Female Hamadryas Baboons After Male Take-Overs: "Deceptive" Swellings as a Possible Female Counter-Strategy Against Infanticide, Am. J. Primatol. 52, 157-168.

Zinner, D., Schwibbe, M. H., \& Kaumanns, W. (1994). Cycle synchrony and probability of conception in female hamadryas baboons Papio hamadryas. Behav. Ecol. Sociobiol. $35,175-183$.

Zinner, D., Nunn, C. L., van Schaik, C. P., \& Kappeler, P. M. (2004). Sexual selection and exaggerated sexual swellings of female primates. In: Kappeler, P. M. \& van Schaik, C. P. (Eds.), Sexual Selection in Primates. Cambridge University Press, Cambridge, pp. 71-89. 



\section{CURRICULUM VITAE}

\section{Personal data}

$\begin{array}{ll}\text { Name: } & \text { Ines Fürtbauer } \\ \text { Date of birth: } & 25.12 .1979 \\ \text { Place of birth: } & \text { Vienna, Austria } \\ \text { Nationality: } & \text { Austrian } \\ \text { Marital status: } & \text { single }\end{array}$

\section{University education}

2007-2011: PhD studies at the Max Planck Institute for Evolutionary Anthropology, Leipzig, Germany, and the Georg-August-University Göttingen, Germany PhD thesis: The socio-endocrinology of female reproductive strategies in wild Assamese macaques (Macaca assamensis)

2006: Graduation to Mag. rer. nat.

1998-2006: $\quad$ Biology studies at the University of Vienna, Austria

Diploma thesis: Behaviour and endocrinology of free-living, maturing, male gibbons (Hylobates lar)

\section{Basic education}

1990-1998: $\quad$ Secondary school in Vienna, Austria

1986-1990: $\quad$ Elementary school in Vienna, Austria 


\section{PUBLICATIONS}

Fürtbauer, I., Schülke, O., Heistermann, M., \& Ostner, J. (2010). Reproductive and life history parameters of wild female Macaca assamensis. Int. J. Primatol. 31, 501-517.

Fürtbauer, I., Heistermann, M., Schülke, O., \& Ostner, J. (submitted). Fecal androgen excretion and fetal sex effects during gestation in wild Assamese macaques (Macaca assamensis). Am. J. Phys. Anthropol.

Fürtbauer, I., Heistermann, M., Schülke, O., \& Ostner, J. (submitted). Concealed fertility and extended female sexuality in a non-human primate (Macaca assamensis). PLoS ONE.

Fürtbauer, I., Mundry, R., Heistermann, M., Schülke, O., \& Ostner, J. (submitted). You mate, I mate: Macaque females synchronize sex not cycles. PLOS ONE. 